\title{
Subcellular distribution of apoptosis associated speck-like protein mediates inflammasome assembly: A novel mechanism in the regulation of interleukin-1 beta release
}

Nicole B. Bryan

West Virginia University

Follow this and additional works at: https://researchrepository.wvu.edu/etd

\section{Recommended Citation}

Bryan, Nicole B., "Subcellular distribution of apoptosis associated speck-like protein mediates inflammasome assembly: A novel mechanism in the regulation of interleukin-1beta release" (2011). Graduate Theses, Dissertations, and Problem Reports. 4701.

https://researchrepository.wvu.edu/etd/4701

This Dissertation is protected by copyright and/or related rights. It has been brought to you by the The Research Repository @ WVU with permission from the rights-holder(s). You are free to use this Dissertation in any way that is permitted by the copyright and related rights legislation that applies to your use. For other uses you must obtain permission from the rights-holder(s) directly, unless additional rights are indicated by a Creative Commons license in the record and/ or on the work itself. This Dissertation has been accepted for inclusion in WVU Graduate Theses, Dissertations, and Problem Reports collection by an authorized administrator of The Research Repository @ WVU.

For more information, please contact researchrepository@mail.wvu.edu. 
Subcellular Distribution of Apoptosis Associated Speck-like Protein Mediates Inflammasome Assembly: A Novel Mechanism in the Regulation of Interleukin-1beta Release

Nicole B. Bryan

\begin{abstract}
Dissertation Submitted to the School of Medicine at West Virginia University

In Partial Fulfillment of the Requirements for the Degree of Doctor of Philosophy in Cancer Cell Biology
\end{abstract}

\author{
Steven Frisch, Ph.D., Chair \\ Barbara Ducatman, MD \\ Dan Flynn, Ph.D. \\ Fred Minnear, Ph.D. \\ Yongyut Rojanasakul, Ph.D. \\ Linda Vona-Davis, Ph.D. \\ Christian Stehlik, Ph.D., Mentor
Cancer Cell Biology Program
Morgantown, West Virginia
2010

Keywords: apoptosis associated speck-like protein (ASC), inflammasome, inflammation, IL-1beta, monocytes, macrophage 


\section{ABSTRACT \\ Subcellular Distribution of Apoptosis Associated Speck-like Protein Mediates Inflammasome Assembly: A Novel Mechanism in the Regulation of Interleukin-1 beta Release}

Nicole B. Bryan

Inflammation is an essential component of the innate immune defense against pathogens. The process of inflammation is mediated by the rapid release of intercellular mediators, which function in the activation and recruitment of cellular components of both the innate and adaptive immune system. However, dysregulation of this process, resulting in sustained inflammation is the underlying cause of a number of common pathological conditions. These include hereditary autoinflammatory disorders, gout, arthritis, asthma, and cancer. Furthermore, IL-1beta is a key component of the tumor microenvironment, which serves to recruit tumor-associated macrophages, promote angiogenesis, enhance NF-kB activation, and suppress anti-tumor immunity. The studies presented here explore novel regulatory mechanisms of IL-1beta maturation through the modulation of the inflammasome adaptor protein ASC. ASC is an intriguing protein, which participates in both inflammatory and apoptotic pathways and is frequently silenced by aberrant methylation in a large number of cancers. It is a small protein, consisting of only two protein-interaction domains, an $\mathrm{N}$ terminal PYD, and a C-terminal CARD. Numerous studies have found that it is required for inflammasome assembly following activation of a number of diverse cytoplasmic NLR receptors. Therefore, regulation of this protein has broad implications for IL-1beta maturation.

In the first study, we examined the endogenous localization of ASC. Several independent research groups had found that ectopic expression of ASC results in several distinct localization patterns including nuclear, cytoplasmic, and the formation of a characteristic perinuclear aggregate termed a 'speck'. However, the subcellular distribution of endogenous ASC had never been evaluated. Therefore, we examined the subcellular localization pattern of ASC in several monocytic cell lines as well as in primary monocytes and macrophages. We discovered that in resting monocytes, ASC is localized diffusely throughout the nucleus. However, upon inflammatory stimulation of the cells, we determined that ASC was rapidly redistributed to the cytoplasm where it subsequently formed a perinuclear aggregate, reminiscent of the 'specks' observed upon overexpression of this protein. Furthermore, we determined that other core inflammasome proteins NLRP3 and caspase- 1 co-localized with ASC in these aggregates, suggesting that they represent inflammasomes. Additionally, we 
found that nuclear export of ASC is necessary for proper inflammasome assembly and processing of IL-1beta.

In Study 2, we identified Mycoplasma sp. as a novel activator of the inflammasome. Infection with Mycoplasma sp. cause a number of organ-specific diseases in humans, and they have also been linked to a number of proinflammatory disorders. Therefore, we hypothesized that IL-beta played a role in the innate immune defense against these pathogens. We discovered that incubation of monocytes and macrophages with either live or heat-killed Mycoplasma sp. stimulated the secretion of mature IL-1beta at levels comparable to those observed with known inflammasome activators. Furthermore, we also determined that Mycoplasma infection induced the nuclear to cytoplasmic translocation of ASC, as well as the formation of perinuclear aggregates, just as we observed with known inflammasome activators in Study 1. Finally, we showed that Mycoplasma-induced release of IL-1beta was dependent upon ASC, further indicating the role of the inflammasome in this process. Based upon this data, we hypothesize that chronic infection with Mycoplasma sp. results in sustained production of IL-1beta, which leads to a prolonged inflammatory state.

In our third and final study, we identified and characterized three ASC isoforms with regards to their subcellular localization, and their ability to function as an inflammasome adaptor protein. ASCb lacks the linker domain, ASCc possesses an in-frame deletion in the PYD, which does not disrupt the linker region or the CARD, and ASCd expresses only the first 35 amino acids of the PYD, which is fused to a novel peptide. Upon ectopic expression, each isoform exhibited a different localization pattern, and none of them formed the classic perinuclear 'speck', which has become the hallmark of full-length ASCa. Upon co-expression with either NLRP3 ${ }^{(\mathrm{R} 260 \mathrm{~W})}$ or caspase-1, co-localization was only observed by those isoforms, which expressed a fully intact PYD or CARD, respectively. Functionally, only ASCb was able to mediate IL-1beta processing when co-expressed with NLRP $3^{(\mathrm{R} 260 \mathrm{~W})}$ and pro-caspase-1, although to a lesser extent, presumably because neither of its protein interaction domains were disrupted.

In summary, these studies show novel mechanisms by which IL-1beta release is regulated and induced. The discovery of novel ASC isoforms and the role its subcellular distribution plays in the regulation of IL-1beta processing provide us with new insights into how inflammasome assembly is regulated and fin-tuned. Furthermore, the nuclear to cytoplasmic redistribution of ASC provides us with a potential new therapeutic target that would prevent the release of IL1 beta without impacting the expression of ASC. 


\section{Acknowledgements}

The past four years have truly been a whirlwind that was both challenging and rewarding. Looking back now, I believe I have come out on the other side having learned how to ask questions as a scientist, and subsequently pursue the answers. However, this journey has not been mine alone. There have been numerous people along the way who have provided invaluable guidance as well as support and encouragement for which I will be eternally grateful.

First, I must begin by thanking Jose and my son Alejandro. You both have loved me so well and filled my life with so much joy that every empty place that ever existed in my life and in my heart is now overflowing. Jose, you are my rock. You give me strength when I have none, keep me whole, and maintain the balance in my life. I am still amazed at the way you always know just what I need, and for those rare quiet moments when you manage to keep the world at bay for me. Alex, watching you learn and grow has been the single greatest joy of my life. I never realized how great my potential for loving some was, until I had you.

Thanks also to my parents, whose unwavering support and faith in me has helped me to make so many dreams come true. Dad, you have helped me to reach higher than I ever thought possible. Mom, you are the epitome of grace and compassion. I can only hope that I will one day be able to alleviate people's suffering as well as I have watched you do so effortlessly over the years.

To my mentor Christian - thank you for your expert guidance, and unlimited patience. I have learned so much from you and Andrea that I will carry with me on my journey toward becoming an independent scientist. I have been so fortunate to have you as my mentor. Dr. Yon, Lu, Neelam, Anand, Hosam, and Djordje, thank you all so much for taking me into your laboratory, and including me in everything you did. You have taught me a lot, and I will truly miss every one of you.

As for our CCB program, I am so grateful to all students present and past, with whom I have shared countless experiences. I would especially like to acknowledge Siera, Kristin (lab tech extrodinaire), Stephanie, and Laura. I am so thankful for the friendship you have each given to me. Over the past four years, you have all enriched my life in so many ways. The CCB program would not have been the same without each one of you, and neither would I.

I would also like to thank Dr Minnear for not letting me ever get too comfortable - Dr. Frisch for always challenging me and offering helpful advice and scientific insights - Dr. Ducatman for helping me maintain my focus on clinical relevance - Dr. Flynn for his dedication and willingness to help me - Dr. Vona-Davis for helping me to maintain a positive outlook, and reminding me that there is no other job I would rather have - and Dr. Martin for her guidance, support, and of course for her microscope expertise.

I would like to dedicate this work to the memory of my sister Natalie Rose Bryan. "What a pearl, at great price, you built in us, Natalie". 


\section{Table of Contents}

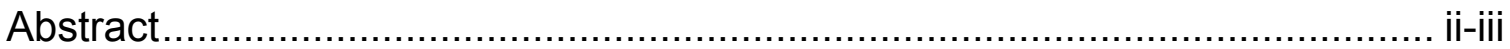

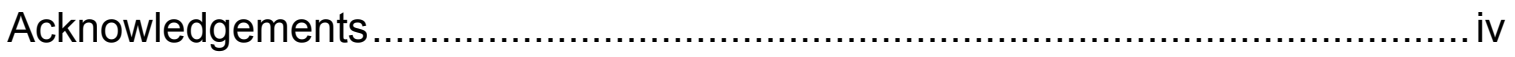

Table of Contents …...............................................................................

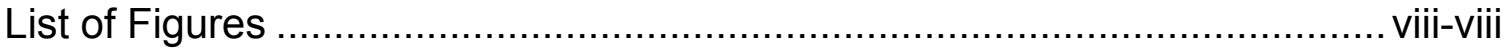

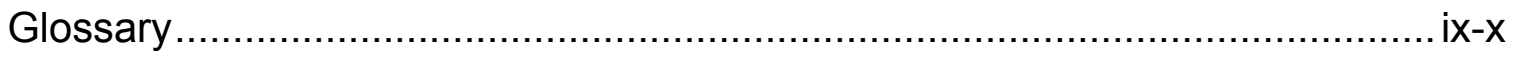

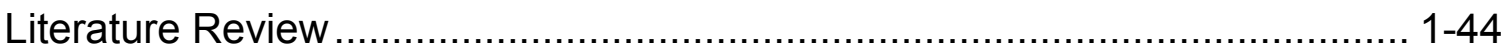
Interleukin-1

The Inflammasome

Regulation of thee Inflammasome

Study 1: Activation of Inflammasomes Requires Intracellular Redistribution of the Apoptotic Speck-like Protein Containing a Caspase Recruitment Domain (ASC). $45-98$

Abstract Introduction

Experimental Procedures

Results

Discussion

Study 2: Mycoplasma: A novel activator of the inflammasome $99-128$

Abstract

Introduction

Experimental Procedures

Results

Discussion 
Study 3: Identification and Characterization of Three Novel ASC Isoforms

Abstract

Introduction

Experimental Procedures

Results

Discussion

General Discussion $161-168$

General References $169-182$ 


\section{List of Figures}

\section{Study 1}

1. Nuclear localization of ASC

2. Cytosolic redistribution of ASC in response to inflammatory stimulation of monocytes

3. Aggregate formation of endogenous ASC in response to inflammatory stimulation in macrophages

4. Aggregation of ASC occurs within one hour of inflammatory stimulation of macrophages and depends upon the continued presence of the stimulus

5. Caspase-1 and NLRP3 co-localize with ASC in aggregates

6. ASC aggregate formation is linked to the maturation of IL-1beta

7. ASC localization to the cytosol is required for inflammasome formation and efficient IL-1beta secretion

8. A model for inflammasome formation and activation

\section{Study 2}

1. Mycoplasma sp induce secretion of mature II-1 beta from THP-1 monocytes.

2. Live infection with Mycoplasma sp. induces nuclear to cytosolic redistribution of ASC in human monocytic cell lines

3. Stimulation of THP-1 macrophages with HKAL results in the formation of perinuclear ASC aggregates 
4. $A S C$ is required for IL-1beta secretion induced by HKAL

5. The mycoplasma lipoprotein FSL1 is sufficient to cause macrophage inflammasome activation.

\section{Study 3}

1. Structural alterations in the protein organization of ASC isoforms

2. Subcellular distribution of ASC isoforms

3. ASC isoforms exhibit different co-localization patterns with other key inflammasome proteins and have a diminished capacity to elicit inflammasome activation.

4. Co-localization of ASC b and ASC $c$ with the full-length ASC a 


\section{Glossary}

$\begin{array}{ll}\text { AIM2 } & \text { absent in melanoma } 2 \\ \text { ASC } & \begin{array}{l}\text { apoptosis associated speck-like protein containing a caspase } \\ \text { recruitment domain }\end{array} \\ \text { ATP } & \text { adeonosine triphosphate } \\ \text { BCA } & \text { bicinchoninic acid } \\ \text { CARD } & \text { caspase recruitment domain } \\ \text { COPs } & \text { CARD only proteins } \\ \text { DAMP } & \text { danger associated molecular pattern } \\ \text { DMEM } & \text { Dulbecco's modified eagle's medium } \\ \text { DTT } & \text { dithiothreitol } \\ \text { ECL } & \text { enzymatic chemiluminescence } \\ \text { EDTA } & \text { ethylenediaminetetraacetic acid } \\ \text { EGTA } & \text { ethylene glycol tetraacetic acid } \\ \text { ELISA } & \text { enzyme-linked immunosorbent assay } \\ \text { EMT } & \text { epithelial to mesenchymal transition } \\ \text { EST } & \text { expressed sequence tag } \\ \text { FADD } & \text { Fas-associated death domain protein } \\ \text { FBS } & \text { fetal bovine serum } \\ \text { GAPDH } & \text { glyceraldehyde-3 phosphate dehydrogenase } \\ \text { GFP } & \text { green fluorescent protein } \\ \text { GST } & \text { glutathione-S-transferase } \\ \text { HBSS } & \text { Hank's balanced salt solution } \\ \text { HKLP } & \text { heat-killed Legionella pneumophilia } \\ \text { HKSA } & \text { heat-killed Staphylococcus aureus } \\ \text { HRP } & \text { horseradish peroxidase } \\ \text { IKK } & \text { IkappaB kinase } \\ \text { IL-1 } & \text { interleukin 1 } \\ \text { LPS } & \text { lipopolysaccharide } \\ \text { LRR } & \text { leucine-rich repeat } \\ \text { MCP1 } & \text { monocytes chemoattractant protein 1 } \\ \text { MMP } & \text { matrix metalloproteinase } \\ \text { NACHT } & \text { neuronal apoptosis inhibitory protein/MCH class II transcription } \\ & \text { activator/incompatibility locus protein from Podospra } \\ & \text { auserina/telomerase-associated protein } \\ \text { NALP } & \text { NACHT and leucine rich repeat protein } \\ \text { NF-kB } & \text { nuclear factor-kappa B } \\ \text { NLR } & \text { nucleotide-binding oligomerization domain-like receptor } \\ \text { NLS } & \text { nuclear localization sequence } \\ \text { Nod } & \text { nucleotide-binding oligomerization domain } \\ \text { NP-40 } & \text { nonidet-P40 } \\ \text { P2X } R & \text { P2X } \text { receptor } \\ & \\ & \end{array}$ 


$\begin{array}{ll}\text { PAGE } & \text { polyacrylamide gel electrophoresis } \\ \text { PAMP } & \text { pathogen associated molecular pattern } \\ \text { PBS } & \text { phosphate buffered saline } \\ \text { PMA } & \text { phorbal 12-myristate 13-acetate } \\ \text { PMSF } & \text { phenylmethylsulfonyl fluoride } \\ \text { POP1 } & \text { PAAD-only protein 1 } \\ \text { POPs } & \text { pyrin only proteins } \\ \text { PRR } & \text { pattern recognition receptor } \\ \text { PVDF } & \text { polyvinylidene fluoride } \\ \text { PYD } & \text { pyrin domain } \\ \text { RFP } & \text { red fluorescent protein } \\ \text { RNA } & \text { ribonucleic acid } \\ \text { SA } & \text { splice acceptor } \\ \text { SD } & \text { standard deviation } \\ \text { SD } & \text { splice donor } \\ \text { SDS } & \text { sodium dodecyl sulfate } \\ \text { ShRNA } & \text { short hairpin RNA } \\ \text { SiRNA } & \text { small interfering RNA } \\ \text { SLO } & \text { streptolysin O } \\ \text { TAM } & \text { tumor associated macrophage } \\ \text { TLR } & \text { toll-like receptor } \\ \text { VEGF } & \text { vascular endothelial growth factor }\end{array}$




\section{Literature Review}

Inflammation is an essential component of the innate immune system, and is required for the elimination of invading pathogens. The process of inflammation is primarily regulated by cytokines, which are secreted by immune cells such as monocytes and macrophages. When functioning properly, inflammation is a key process in protection against viruses, bacteria, fungi and protozoa. However, excessive production of inflammatory cytokines can inflict a large amount of unwarranted damage on an individual. This occurs in immunelinked diseases, such as autoinflammatory disorders, which are characterized by

recurrent, unprovoked bouts of fever and inflammation, with no apparent B cell or T cell involvement.

\section{Interleukin-1}

Interleukin-1 (IL-1) is a key cytokine in the inflammatory process. It is distinguished from other cytokines by the fact that it can initiate a response in nearly every cell type, and therefore its effects are not limited to immune cells. This fact, in combination with the ability of IL-1 signaling to result in the upregulation of numerous other pro-inflammatory cytokines has led many people to consider it to be a 'master cytokine'. The IL-1 gene family consists of three members: IL-1alpha, IL-1 beta, and IL-1 receptor antagonist (IL-1Ra). Of these, only the IL-1Ra, which elicits no response upon interaction with the IL-1 receptor, possesses a signal sequence that enables it to be secreted from the cell. IL- 
1alpha and IL-1 beta function as agonists when bound to the IL-1 receptor, and display similar biological activity upon release from the cell. However, they differ in their intracellular functionality, their processing from the pro-form to the active cytokine, and the circumstances under which they are released. IL-1beta is only synthesized under conditions of cell stress or pathogenic invasion, remains inactive while in its full-length form, and exhibits no intracellular activity. IL1alpha, on the other hand, is frequently expressed in cells, is active in both its cleaved and its pro-form, and is believed to exert functions in the nucleus related to cell differentiation. In addition, IL-1 beta requires activation of caspase-1 in order to be cleaved into its active $17.5 \mathrm{kD}$ form, whereas IL-1alpha is processed by membrane associated calcium-dependent calpains. Furthermore, IL-1beta is rapidly synthesized and released under conditions of pathogenic challenge, while IL-1alpha is usually only released in the context of severe cell stress or necrosis. Therefore, elevated serum levels of IL-1alpha are associated with a more devastating disease state than elevated levels of IL-1beta (Dinarello, 1996).

IL-1beta is a very powerful pro-inflammatory cytokine that can exert both local and systemic effects. Upon its release, it can function as a chemoattractant for a number of immune cells including neutrophils, monocytes and macrophages, as well as B and T lymphocytes. Systemically, it is an extremely potent pyrogen, inducing fever at $\mathrm{ng} / \mathrm{kg}$ concentrations in healthy human subjects. Furthermore, upon binding to the IL-1 receptor (IL-1R1), IL-1 $\beta$ can induce downstream signaling, which results in the transcriptional upregulation of 
itself as well as other inflammatory cytokines, including TNF-alpha (Dinarello CA, 1987,Warner, 1987). IL-1beta has become a very important cytokine in the clinical setting as dysregulation of its secretion has been associated with a number of inflammatory disorders including arthritis, asthma, inflammatory bowel disease, ulcerative colitis, atherosclerosis, peridontitis, type 2 Diabetes, lung fibrosis, multiple sclerosis, Alzheimer's disease, stroke, septic shock, periodic fever syndromes such as Familial Mediterranean Fever, and cancer (Barksby HE, 2007,Braddock M, 2004,Samuels, 2006).

A further indication of its key role in inflammatory processes is the extent to which IL-1 $\beta$ signaling is regulated. IL-1-beta is synthesized as a $31 \mathrm{kD}$ precursor, which must be processed into its active $17.5 \mathrm{kD}$ form by either active caspase-1 or extracellular proteases. Activation of this cytokine is believed to occur in a two-step process. First, activation of Toll-like receptors stimulated activation of the transcription factor NF-kappa B. NF- kappa B regulates a large number of pro-survival as well as pro-inflammatory genes, and among them is the gene for pro-IL-1 beta. However, TLR signaling alone is insufficient for complete activation of IL-1 beta. The second step of the process requires the activation of caspase-1 in order to convert pro-IL-1 beta into its active, mature form, which is subsequently secreted from the cell. Thus, there are two distinct requirements for activation of IL-1 beta, which must both be fulfilled in order for the active cytokine to be released from the cell, although under certain conditions 
other inflammatory caspases, such as caspase-5 (human) or caspase-11 (mouse), are required for IL-1beta processing (Netea MG, 2008). Intriguingly, the mechanism of the release of IL-1alpha and IL-1beta has still not been fully elucidated. Both IL-1 beta and IL-1 alpha lack a signal sequence, and neither has been found to localize to the ER, or to the Golgi apparatus, and inhibition of trafficking in these membrane systems had no impact on the release of IL-1 beta. Furthermore, Rubartelli et al., demonstrated that viable cells are required for release of the active form of IL-1 beta, and that only pro-IL-1 beta is released upon cell lysis, suggesting IL-1 is released by a nonclassical secretion pathway (A Rubartelli, 1990). Recently, a study has emerged implicating caspase-1 as the key regulator for the secretion of both IL-1 beta and IL-1 alpha as well as some other proteins known to be secreted by in a nonclassical manner. These researchers discovered that the secretion both IL-1 beta and IL-1 alpha, which is not a substrate for caspase-1, was impaired in macrophages derived from caspase $-1^{-/-}$mice. Furthermore, they showed that the enzymatic activity of caspase-1 is required in order for it to mediate protein secretion, and that loss of NALP3, and ASC also resulted in a decrease in IL-1 alpha secretion. Finally, this group identified other leaderless secreted proteins, whose secretion may be regulated by caspase-1 including FGF-2, which functions in angiogenesis and tissue repair (Keller M, 2008). These data suggest that caspase-1 may have an additional role in the regulation of inflammation and wound repair, distinct from its cleavage of pro-IL-1 beta. 
Once active IL-1 has been released from the cell, there are further mechanisms to mitigate its signaling potential. First, there are two potential IL-1 receptor proteins, IL-R1, IL-1R2, and a soluble, shedded IL-1R1. Only the IL-R1 possesses a cytoplasmic tail, which can transmit downstream signaling upon binding to IL-1-beta. When IL-1-beta binds to IL-1R1, it is initially a low affinity interaction. However, a conformational change occurs in the receptor, which enables it to subsequently bind to the IL-1 accessory protein (IL-1R AcP), which results in a higher affinity interaction and is necessary for transmission of downstream signaling. The IL-1R2 and the soluble IL-1R1, on the other hand, lack the cytosolic domain and functionally serves as a decoy receptor, though its initial binding to IL-1 $\beta$ results in a more stable interaction. Cells expressing the IL1 receptors will express ten times more of the non-signaling IL-1R2 than IL-1R1 as an additional safeguard against unwarranted IL-1 signaling.

Circulating monocytes and adherent macrophages serve as major producers of IL-1 beta. Monocytes are circulating blood cells produced in the bone marrow by hematopoeisis. They are a member of the myeloid lineage of blood cells, and play a crucial role in innate immunity. Macrophages are derived from circulating monocytes and are found in numerous tissues throughout the body. They arise through the differentiation of monocytes, which occurs when they extravasate from the vasculature and enter the tissue spaces. They are distinguished from monocytes by the fact that they are much larger and adherent. In addition, many tissues, which frequently encounter pathogenic challenges, 
such as the lungs and GI tract, maintain a resident population of macrophages. Both monocytes and macrophages possess phagocytic abilities and can serve as antigen presenting cells for cytotoxic and helper T cells. Furthermore, they are both capable of secreting numerous inflammatory cytokines, which can function as chemotactic factors for other innate as well as adaptive immune cells.

However, for many years it has been known that monocytes and macrophages exhibit differential requirements in order to secrete active IL-1 beta. Specifically, numerous studies with macrophages demonstrated a requirement for extracellular ATP in addition to bacterial ligands such as LPS. No such requirement has been documented for monocytes. Furthermore, monocytes are capable of secreting larger amounts of IL-1 beta in comparison with macrophages. Fortunately, recent evidence has emerged that may begin to explain these differences, which have long been a mystery to researchers. First, the Rubartelli lab demonstrated that monocytes could secrete ATP in response to several different bacterial ligands as well as uric acid. The amount of mature IL-1 beta and IL-18 secreted by these cells was closely correlated with the amount of ATP they released. Therefore, they proposed that the ATP secreted by monocytes can act in an autocrine manner in order provide the additional stimulation required for IL-1 beta and IL-18 release. Data, which showed that blocking the P2X7 receptor, which binds extracellular ATP, prevents release of the mature cytokines, but not intracellular accumulation of the precursor forms, provided support for this hypothesis (Piccini A, 2008). Additional studies in the 
Dinarello laboratory confirmed these results and also provided new insights. They found that monocytes constitutively express the active $\mathrm{p} 10$ subunit of caspase- 1 in addition to the inactive p45 precursor, while macrophages only express the inactive precursor. Furthermore, they determined that prevention of adherence of differentiated monocytes resulted in maintenance of their ability to secreted active IL-1 beta in the absence of ATP (Netea MG, 2009).

The process of inflammation is intimately intertwined with those involved in the development and progression of cancer. First, many cancers develop at sites of chronic inflammation or infection. Among them are gastric cancer, which is strongly linked to chronic infection with the bacterium H. pylori, and colon cancer, which often develops in the setting of inflammatory bowl disease. In addition, a large portion of the tumor microenvironment is composed of inflammatory cells. Numerous studies have demonstrated the importance of the microenvironment in tumor survival, proliferation and metastasis. Macrophages are one of the essential inflammatory cell components in the tumor microenvironment. They are frequently recruited to the tumor site by tumor cell mediated secretion of the cytokine monocytes chemoattractant protein 1 (MCP1). Once recruited to the site, tumor associated macrophages (TAMs) are capable of either inhibiting or promoting tumor growth. TAMs can also aid in the process tumor invasion through the stimulation of angiogenesis and by secreting matrix metalloproteinases (MMPs), which degrade the extracellular matrix to enable tumor cell invasion (Ono, 2008). 
The role of inflammatory cytokines is increasingly being evaluated in the context of cancer. Evidence has accumulated, which indicates that an appropriate balance of inflammatory cytokines must be present in order for tumor development to occur. In the presence of excessive inflammation, tumor regression will occur. However, too few cytokines present will restrict tumor growth. While the necessity of proinflammatory cytokines for tumor progression appears counterintuitive at first glance, a deeper look reveals that a chronic inflammatory state effectively provides a fertile environment, which can nurture tumor growth. Many of the cellular processes stimulated by pathogenic invasion or wound healing are also advantageous to neoplastic cells. Inflammatory cytokines induce proliferation, recruit inflammatory cells, and also stimulate an increase in the production of reactive nitrogen and oxygen species, which can cause DNA damage. This DNA damage can be further exacerbated by cytokines, which can overcome p53 regulatory control. Thus, chronic inflammation results in an environment in which cell proliferation is enhanced, there is an increase in DNA damage, and normal cell cycle controls are bypassed, all of which also enhance tumor development (Coussens LM, 2002).

Recently, IL-1beta has emerged as a key inflammatory player in tumor progression and invasiveness. It is often secreted by TAMs and elevated levels of IL-1beta in the tumor microenvironment result in an increase in the production of angiogenic factors including VEGFA, and IL-8. A recent study documented that in IL-1beta deficient mice, there was decreased rate of chemically-induced 
tumorigenesis, less leukocytic infiltrate surrounding tumors, and decreased invasiveness (Krelin, 2007). Furthermore, cancer cells frequently acquire the ability to secrete IL-1beta, which can act synergistically with the IL-1 produced by the tumor microenvironment to enhance tumor growth and invasiveness (Kimura YN, 2007).

One tumor setting in which increasing evidence has emerged for the critical importance of IL-1beta signaling is gastric cancer. In one recent study, treatment of several gastric cancer cell lines with IL-1 beta was found to induce methylation of the E-cadherin gene, which is an important event in the development of gastric carcinogenesis (Qian X, 2008). Downregulation of Ecadherin is an essential step in the process of epithelial to mesenchymal transition (EMT), which is important for tumor cell invasion and metastasis. Tu et al recently generated an IL-1beta tissue specific transgenic mouse, which they utilized to demonstrate that chronic overexpression of IL-1beta in the stomach is sufficient for the development of metaplasia, and even high grade dysplasia/carcinoma in the absence of $H$. pylori infection. Furthermore, they found that expression of IL-1beta could recruit myeloid derived suppressor cells to the tumor site. These cells have been found to enhance tumor progression and invasion in a manner similar to TAMs in addition to their ability to suppress B and T cell proliferation (Tu S, 2008). 


\section{The Inflammasome}

In recent years, evidence has accumulated in favor of an activation platform serving as the site of activation of IL-1beta. This platform is composed of a multi-protein aggregate and is referred to as the inflammasome. The initial description of the inflammasome included the proteins caspase-1, caspase-5, ASC, and the cytosolic receptor NLRP protein NALP-1 (Bruey, 2004,Martinon, 2002). Furthermore, ASC was determined to be an essential component of the inflammasome in order to achieve activation of caspase- 1 and caspase- 5 . Additionally, NLRP3 (Cryopyrin) was also identified as a participant in inflammasome formation (Kanneganti, 2006b,Mariathasan, 2006,Martinon, 2006,Sutterwala, 2006). However, an inflammasome containing NLRP3 could only stimulate caspase- 1 activation, not caspase- 5 (Agostini, 2004). The essential role of ASC in formation of these complexes was further solidified by the generation of ASC-null macrophages and mice. The absence of ASC severely diminished the ability of the macrophages to secrete IL-1 $\beta$ or to respond to intracellular pathogens. In the mice, the absence of ASC prevented the development of endotoxic shock in response to LPS (Mariathasan, 2004,Yamamoto, 2004).

Inflammasome assembly is initiated when the ligand-sensing region (LRR domain) of a cytosolic NLR receptor recognizes a specific molecular pattern. These molecules may be derived from conserved regions of invading pathogens, such as bacterial lipopolysaccharide (LPS) or peptidoglycan. In addition, certain 
endogenous molecules such as ATP and uric acid can also be recognized as danger signals. The recognition of a specific ligand is then followed by the recruitment of the adaptor protein ASC via a homotypic PYD-PYD interaction between the $\mathrm{N}$-terminal domain of $\mathrm{ASC}$ and the $\mathrm{N}$-terminal protein interaction domain of the receptor. Once recruited, ASC subsequently recruits the inactive pro-caspase-1 via a homotypic CARD-CARD interaction. Recruitment of multiple pro-caspase-1 molecules results in their activation via the induced proximity mechanism.

NLRP Receptor

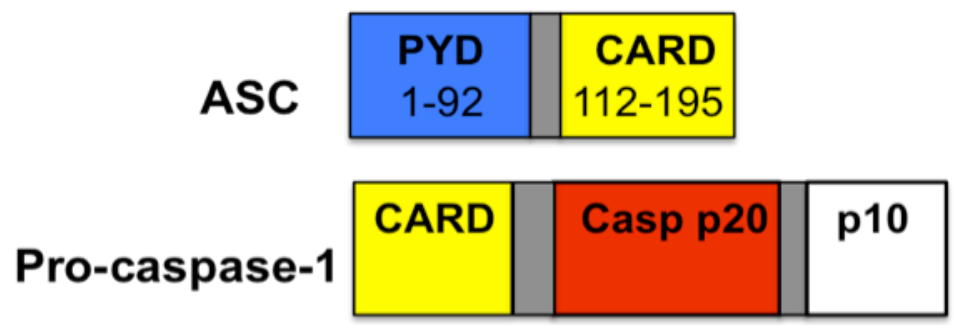

Schematic 1: The linear structure of key inflammasome proteins. The cytosolic NLRP receptor consists of an N-terminal protein interaction domain (PYD), a nucleotide-binding oligomerization domain (NACHT), and a c-terminal ligand-sensing domain (LRR). The adaptor protein ASC is recruited to the NLRP receptor via homotypic PYD-PYD interaction. ASC then uses its CARD domain to recruit pro-caspase-1 via homotypic CARD-CARD interaction. Pro-casapse-1 molecules then undergo autocatalytic processing generating the active form of the caspase with $\mathrm{p} 20$ and $\mathrm{p} 10$ subunits (red and white). PYD = PYRIN domain (blue), $\mathrm{NACHT}=$ neuronal apoptosis inhibitory protein (NAIP)/MHC class II transcription activator (CIITA)/incompatibility locus protein from Podospora auserina (HET-E)/telomerase-associated protein (TP1) (orange), LRR = leucine rich repeat (green), CARD = caspase recruitment domain (yellow). 
The NLR family of proteins function as cytosolic pattern recognition receptors, which recognize specific microbial pathogen associated molecular patterns (PAMPs) as well as endogenous danger associated molecular patterns (DAMPs). Structurally, these proteins consist of an $\mathrm{N}$-terminal protein interaction domain, which is frequently either a PYD or a CARD domain, a central nucleotide binding NACHT domain, and a C-terminal region of leucine rich repeats (LRR). To date, more than twenty cytosolic NLR receptor proteins have been identified. Of these, fourteen possess a PYD domain at the N-terminus, which functions as their protein interaction domain. The receptors studied thus far, even those with a CARD, require the adaptor protein ASC in order to recruit and activate caspase-1. Upon recognition of a specific PAMP or DAMP by the LRR domain, the receptor then undergoes an ATP-dependent oligomerization, which results in the unmasking of the protein-interaction domain thereby enabling it to recruit the adaptor protein ASC, or in some instances, caspase-1 directly (Faustin B, 2007). Interestingly, in addition to serving as the recognition domain the LRR domain of the NLR receptors is also instrumental in preventing unwarranted inflammasome activation, presumably by masking the protein-interaction domain. Deletion of the LRR domain of NLR proteins results in constitutive inflammasome activation and IL-1 beta secretion in the absence of any activation by pathogens or endogenous danger signals (Faustin B, 2007).

NLRP3 /cryopyrin is the most studied and best characterized member of the NLR family. Missense mutations in the NACHT domain of this receptor result 
in the hereditary autoinflammatory disorders collectively known as the cryopyrinopathies, which include a spectrum of diseases, ranging in severity. The specific NLRP3 diseases are familial cold autoinflammatory syndrome (FCAS), which is the mildest form, Muckle-Wells syndrome, and chronic infantile neurologic cutaneous and articular (CINCA) syndrome, which is the most severe (Kastner, 2005,Tschopp, 2003). The mutations associated with these diseases result in constitutive activation of NLRP3, which subsequently recruits ASC on a continuous basis. This results in sustained activation of the inflammasome and IL-1 $\beta$ secretion (Agostini, 2004,Dowds, 2004,Masumoto, 2003,Yu, 2006). The mutations commonly associated with these diseases are an arginine to tryptophan substitution at residue 260 (R260W), an aspartic acid to asparagine substitution at residue 303 (D303N), and a tyrosine to cysteine substitution at residue 570 (Y570C). The R260W mutation has been identified in both FCAS and MuckleWells syndrome. The D303N mutation is observed in Muckle-Wells syndrome and CINCA, while the Y570C mutation is seen in the most severe cases of CINCA.

Interestingly, the NLRP3 mutations, which are associated with increased inflammasome activation, have also been found to induce cell death as well. The Miyachi lab demonstrated that transfection of the common NLRP3 mutants, but not the wild type, into the monocytic THP-1 cell line resulted in cell death. The cell death was apparent shortly after gene expression, as measured by Annexin $\mathrm{V}$ staining. Further analysis revealed that the cell morphology was indicative of a 
necrotic type death, and was dependent upon the release of the lysosomal protein cathepsin B and accompanied by mitochondrial damage. Furthermore, cell death was also observed in THP-1 cells transfected with wild-type NLRP3 following treatment with the NLRP3 specific activator R837 (Fujisawa A, 2007). That the cell death induced by NLRP3 mutants exhibited a closer resemblance to necrosis rather than apoptosis is fitting with their role in autoinflammatory disorders. Necrotic cell death is frequently associated with a local inflammatory response and recruitment of phagocytic cells, whereas apoptosis is not. Additionally, upon pathogenic invasion, cells will frequently favor a necrotic death as a mechanism to elicit a more robust immune response.

To date, several ligands have been identified, which activate the NLRP3 inflammasome. Among them are several pathogen-derived molecular patterns as well as endogenous danger signals. They include bacterial RNA (Kanneganti, 2006b), double-stranded viral RNA (Kanneganti, 2006a), muramyl dipeptide (Marina-García N, 2008,Martinon, 2004), gout-associated uric acid crystals (Martinon, 2006), extracellular ATP (Mariathasan, 2006), $\mathrm{K}^{+}$efflux (Pétrilli V, 2007), silica (Cassel, 9035), asbestos (Dostert, 2008), ROS (Cruz CM, 2007), influenza A (Thomas PG, 2009), and amyloid beta (Halle A, 2008). The number of ligands capable of triggering the NLRP3 inflammasome is particularly intriguing given that many of the known NLR receptors have yet to be associated with a specific ligand. 
The precise mechanism by which ligands activate NLR receptors has not been fully elucidated as a physical interaction between an NLR protein and a pathogenic molecular pattern has never been demonstrated. Therefore, it is possible given the large number of diverse ligands identified for NLRP3 and NLRC4, that the receptor is indirectly activated by a common alteration in endogenous molecules rather than through a direct interaction with the agonists. However, one requirement for NLRP3 and NLRC4 receptor activation that is well established is the transport of the activating ligand to the cytoplasm (Juhas M, 2008,Kanneganti TD, 2007). Some pathogens encode genes for pore-forming toxins, such as Streptococcus, which enable the transport of exogenous substances into the cytoplasm (Marriott HM, 2008). Other pathogens, such as Salmonella and Legionella possess Type III or Type IV secretion systems, which enable them to deliver bacterial components to the cyotosol once they have been phagocytosed (Galán JE, 2006,Juhas M, 2008). Mutational studies with these bacteria have demonstrated that these systems are essential for caspase- 1 and IL-1beta activation. For those pathogens that lack these capabilities, a cytoplasmic delivery mechanism has been identified, which involves activation by ATP, and is closely associated with activation of NLRP3. 


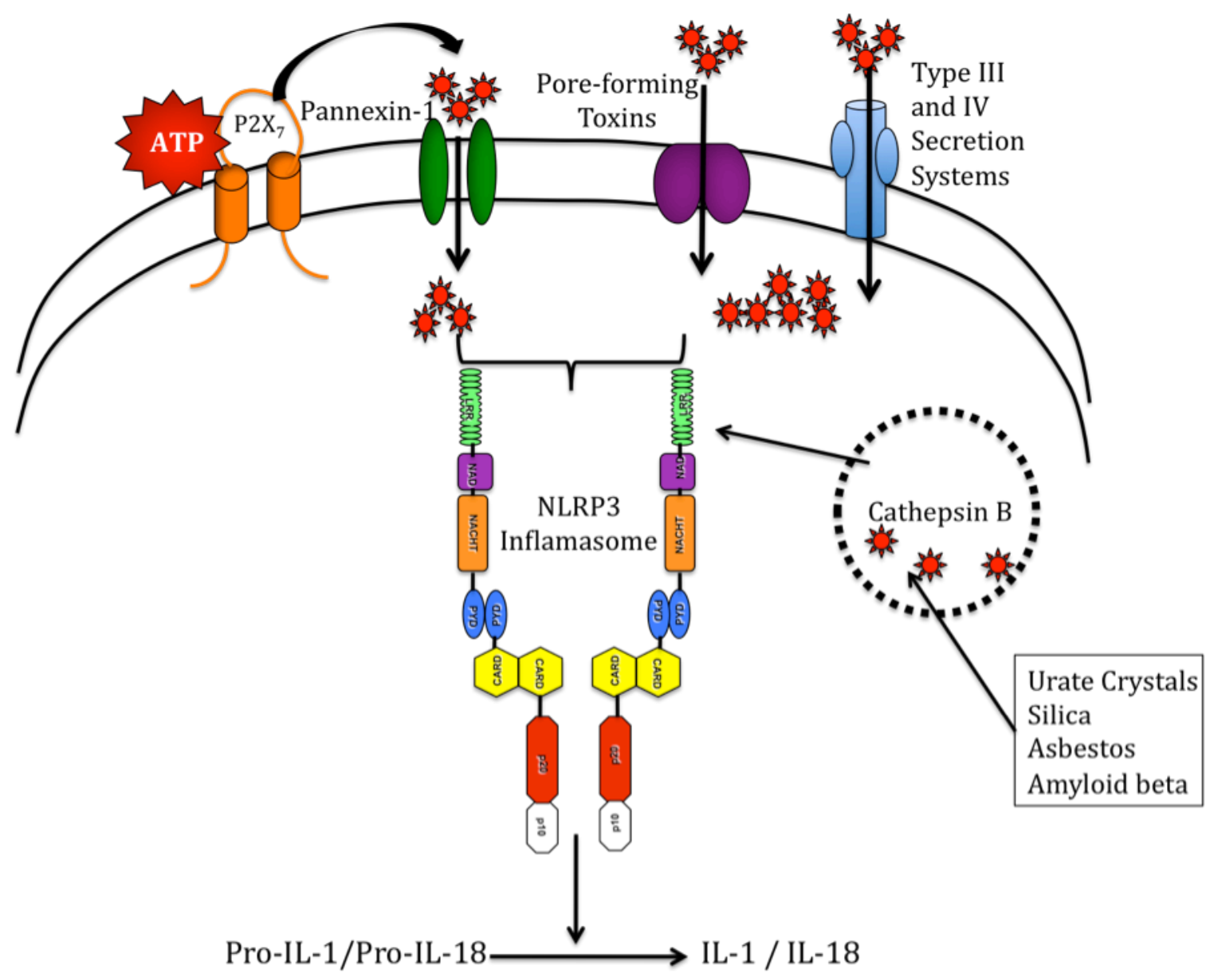

Schematic 2. Mechanisms of cytoplasmic delivery of NLRP3 activating ligands. Inflammasome activation requires the cytoplasmic delivery of the activating ligands. This can be achieved by pathogen-encoded pore-forming toxins or secretion systems. Alternatively, cytoplasmic delivery can be mediated by the opening of membrane channels. The $\mathrm{P}_{2} \mathrm{X}_{7}$ receptor is activated upon binding of extracellular ATP, which functions as a DAMP, and subsequently enables the rapid loss of intracellular $\mathrm{K}^{+}$and the opening of the large membrane channel, pannexin-1. The opening of the pannexin-1 channel allows the delivery of pathogenic ligands. In addition, the phagocytosis of undigestable materials, such as asbestos, causes phagosomal destabilization resulting in the cytoplasmic delivery of cathepsin B, which functions as an NLRP3 ligand.

ATP, which serves as a particularly potent endogenous danger signal, is an interesting activator of the inflammasome. Extracellular ATP binds to the 
purigenic $\mathrm{P} 2 \mathrm{X}_{7}$ receptor and gene ablation studies in mice have demonstrated that it is required for the release of IL-1 beta in response to ATP (Solle M, 2001). When activated, this receptor functions as a cation channel, which results in the immediate efflux of intracellular potassium. In addition, stimulation of this receptor also causes the gradual opening of a larger pore, which is permeable to molecules up to $900 \mathrm{Da}$ in size. Recent studies have identified that the opening of this larger pore is mediated by the hemichannel protein pannexin-1 (Locovei S, 2007). Pelegrin et al demonstrated that the $P 2 X_{7}$ receptor and pannexin-1 are frequently co-expressed in cells and are found co-localized in the cell membrane. Furthermore, they showed that knock-down of pannexin-1 prevented dye uptake by cells in response to ATP activation as well as activation and release of IL1beta, but had no impact on potassium efflux. Finally, they also showed that overexpression of pannexin-1 in $\mathrm{P} 2 \mathrm{X}_{7}$ receptor-null cells caused an increase in the cellular uptake of ethidium, which was not observed in cells that expressed the $\mathrm{P} 2 \mathrm{X}_{7}$ receptor. This evidence suggests that the $\mathrm{P} 2 \mathrm{X}_{7}$ receptor functions as an inhibitor of pannexin-1-mediated pore formation in resting cells, and that this inhibition is relieved upon binding of ATP to the $P 2 X_{7}$ receptor (Pelegrin $P, 2006$ ).

Recent evidence suggests that pannexin-mediated pore formation may serve as a delivery mechanism, which results in the activation of NLRP3. The Nunez laboratory demonstrated that NLRP3 and ASC are required for activation of caspase-1 in macrophages stimulated with a panel of heat-killed bacteria and ATP. Furthermore, they found that incubation of macrophages with heat-killed 
bacteria in the presence of the pore-forming toxin streptolysin O (SLO) could bypass the requirement for ATP and generate active caspase-1 in a NLRP3dependent manner. Finally, this group was able to restore caspase-1 activation by Salmonella, Listeria, and Franciscella, which were deficient in their respective pore-forming toxins or secretion systems, by incubating them with macrophages in the presence of ATP. Furthermore, this activation was dependent upon both pannexin-1 and NLRP3 (Kanneganti TD, 2007 ). Subsequent studies have provided further support for the intracellular delivery of bacterial products mediated by pannexin-1. Muramyl dipeptide (MDP) is a component of peptidoglycan. Recent evidence has emerged, which show that this molecule is taken up by bone marrow derived macrophages (BMDM), and that it localizes in acidified vesicular structures. Stimulation with ATP causes a rapid redistribution of MDP from these compartments to the cytoplasm. Inhibition of pannexin-1 abrogated this localization change. Furthermore, caspase-1 activation was absent in macrophages derived from NLRP3-null mice in response to MDP and ATP stimulation. These data provide additional evidence that the pore formed by pannexin-1 delivers pathogenic molecular patterns to the cytoplasm resulting in the activation of the NLRP3 inflammasome (Marina-García N, 2008).

New evidence has emerged, which links aberrant secretion of IL-1beta to other clinically relevant disease processes. Recently, activation of the NLRP3 inflammasome has been implicated in the pathogenesis of the occupational pneumoconioses silicosis and asbestosis. Studies have demonstrated that 
alveolar macrophages secrete IL-1 beta in response to treatment with silica crystals in a NLRP3, ASC, and caspase-1 dependent manner. Furthermore, gene ablation studies for each of these proteins resulted in a decrease in lung inflammation and fibrosis, indicating that inflammasome activation is necessary for the development of this disease (Cassel SL, 2008). Additional studies have revealed that the mechanism behind the involvement of the inflammasome in the development of silicosis, and other crystals is destabilization of phagasomes. The Latz laboratory has shown that phagocytosis of silica crystals results in a rupture of lysosomes, which then leak their contents into the cytoplasm of the cell. Their evidence suggests that NLRP3 recognizes the enzymes, such as cathepsin B, normally contained within these membrane-bound structures as a danger signal and initiates downstream activation of caspase-1 (Hornung $\mathrm{V}$, 2008). 


\section{NALP3 Inflammasome}

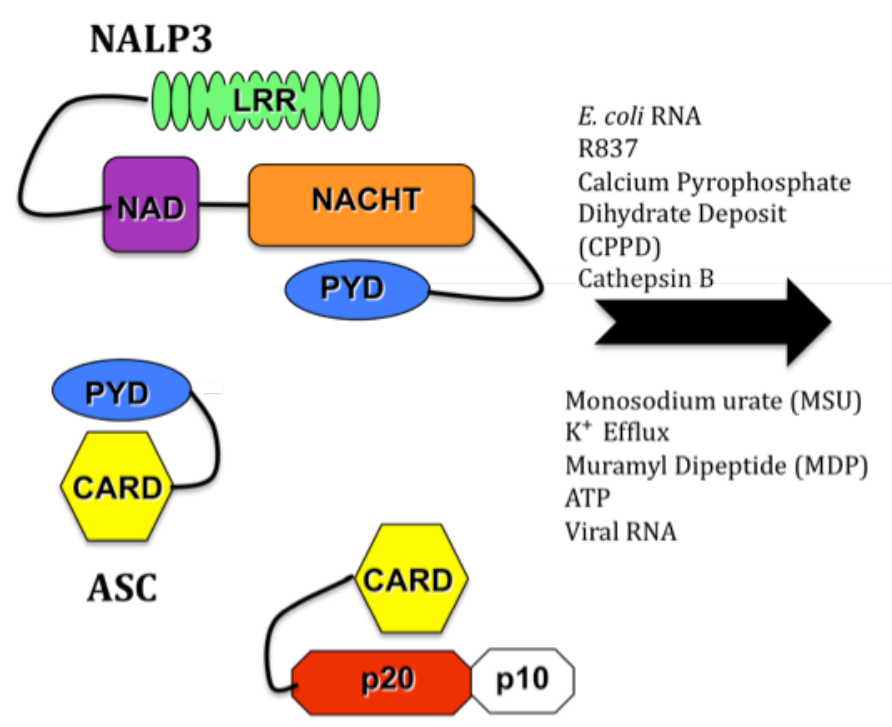

Caspase-1

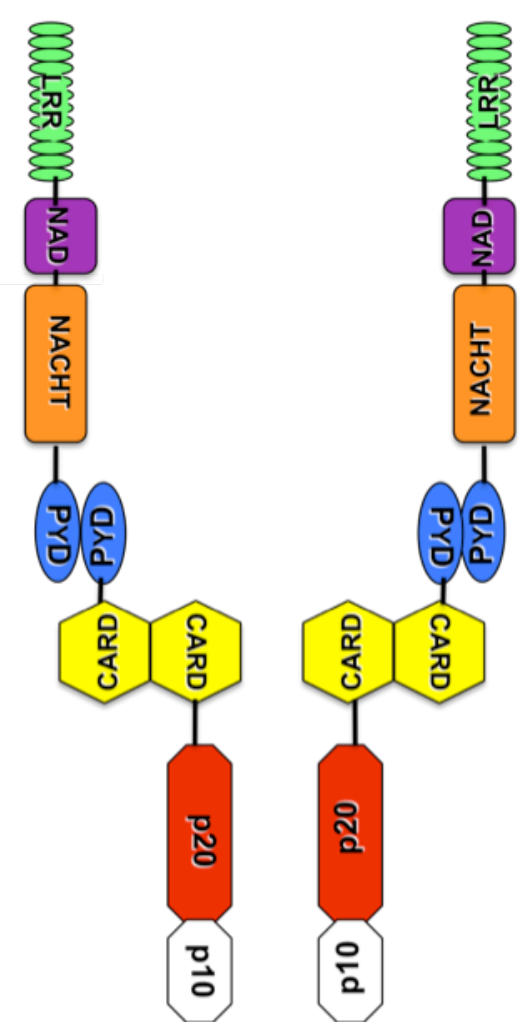

Schematic 3: Activation of the NLRP3 inflammasome. Recognition of several identified ligands by the LRR domain of NLRP3 triggers assembly of the inflammasome. NLRP3 undergoes an ATP-dependent conformational change, which enables it to utilize its PYD to recruit the adaptor protein ASC. ASC subsequently recruits pro-caspase- 1 via CARD-CARD interaction. Once multiple pro-caspase-1 molecules are brought into close proximity, they are then able to process each other and release the catalytically active p10 and p20 subunits from the pro-domain. Active caspase-1 molecules can then cleave $31 \mathrm{kD}$ pro-IL-1 beta into its active $17 \mathrm{kD}$ form, which is secreted by the cell.

The most recently identified inflammasome ligand is cytoplasmic DNA.

The ability of double-stranded DNA to trigger inflammasome assembly and subsequent IL-1beta maturation was first discovered by the Tschopp laboratory in 2008. This group determined that infection with adenovirus, which contains a 
double-stranded DNA genome, elicited secretion of IL-1beta in a NLRP3 and ASC-dependent manner. Intriguingly, they also observed inflammasome activation in response to mammalian DNA, when it was introduced into the cytoplasm. Although the response to mammalian DNA was also ASCdependent, it occurred independently of NLRP3, suggesting the existence of an alternative sensor molecule (Muruve DA, 2008). At the beginning of 2009, this receptor was identified by three independent groups, who determined it to be AIM2 (absent in melanoma 2). AIM2 possesses both a PYD and a HIN200 domain, which is a known nucleotide-binding domain. The presence of these two domains in its structure renders AIM2 capable of interacting directly with both the inflammasome adaptor, ASC and DNA, respectively. AIM2 is unique among previously described receptors in that there is evidence for its direct interactin with its ligand. AIM2 was found to activate both caspase-1 as well as NF-kB in an ASC-dependent manner. Additionally, knock-down of this protein abrogated caspase-1 activation and subsequent IL-1beta secretion in response to mammalian DNA. These data indicate a role for AIM2-mediated inflammasome activity in the innate defense against viral pathogens as well as autoimmune disorders (Burkstummer, 2009, Hornung, 2009, Fernandes-Alnemeri, 2009).

The key inflammasome adaptor protein ASC (ㅁpoptosis associated

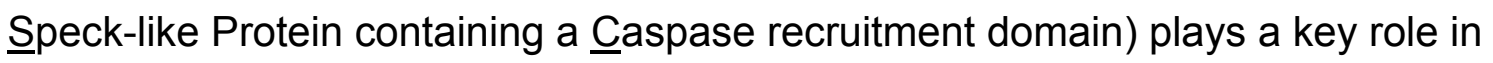
both apoptosis and inflammation. It was initially discovered by two independent research groups. The Sagara lab identified it in HL-60 cells subsequent to 
treatment with the DNA-damaging agent (Masumoto, 1999). In this study, it was noted that ASC formed characteristic perinuclear aggregates in response to the chemotherapeutic agent and that its structure included a caspase recruitment domain (CARD) on the c-terminal end. The second research group identified ASC as a target of methylation-induced silencing in breast cancer and thus was also named Target of Methylation-induced Silencing-1 (TMS1) (Conway, 2000).

Structurally, ASC is a member of the Death Domain Fold Superfamily consisting of two death domain fold domains. A PYD, the newest addition to the death domain fold family, is located at the N-terminus of ASC, and its structure most closely resembles that of the death effector domain (DED) of human FADD (Liepinsh, 2003). A CARD is located at the C-terminus, and a short linker domain joins it to the PYD. While ASC has not been isolated and analyzed by $\mathrm{x}$ ray crystallography, its PYD has been evaluated using nuclear magnetic resonance (NMR) spectroscopy. Like other members of the death domain family, the PYD of ASC consists of six alpha helical folds joined together by loop regions. Further analysis of revealed that the ASC PYD is very bipolar in that most positively charged amino acid residues reside in helices two and three, while the negatively charged ones reside in helices one and four. Based upon this information, it is predicted that the PYD of ASC is likely to interact in a homotypic interaction with other PYD-containing proteins via a type I interaction where helices two and three of one PYD interact strongly with helices two and three of a second PYD (Liepinsh, 2003). 
Shortly after its initial discovery, ASC expression was assessed in a number of normal human tissues by immunohistochemistry, and was found to be present in several tissues including the kidneys, liver, colon, spinal cord, placenta, and most significantly in monocytic cells (Masumoto, 2001b). Conversely, ASC was not observed in cardiac muscle, ciliated epithelium of the trachea, or in lymphocytes. The group also demonstrated that the localization of GFP-tagged ASC as well as each of its component domains upon overexpression. Their studies revealed that both the PYRIN domain and the CARD domain are required in order for the characteristic aggregates (specks) to form. Expression of the individual domains of ASC, on the other hand resulted in formation of long filamentous structures (Masumoto, 2001a). These structures are reminiscent of the "death filaments" previously characterized as occurring upon overexpression of individual members of the death domain fold family (Siegel, 1998). Subsequent studies of the PYD of ASC revealed that amino acids 1-90 are sufficient in order for these filaments to form, and that they do not associate with the cytoskeleton, but rather are formed by self-oligomerization. In addition, positive charge at amino acid residues $\mathrm{K} 21, \mathrm{~K} 26$, and $\mathrm{R} 41$, as well as a negative charge at residues D48 and D51 are crucial for filament formation (Moriya, 2005). Furthermore, the Sagara lab also demonstrated that the PYD and CARD are capable of binding to each other when co-expressed individually. These findings have since been independently confirmed by other groups (Stehlik, 2003b). 
Full Length ASC

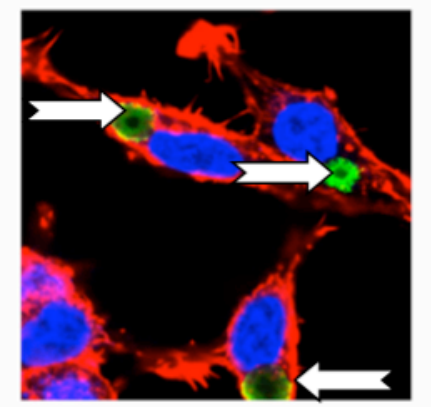

ASC CARD

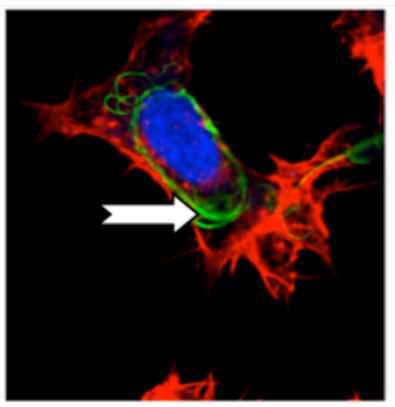

ASC PYD

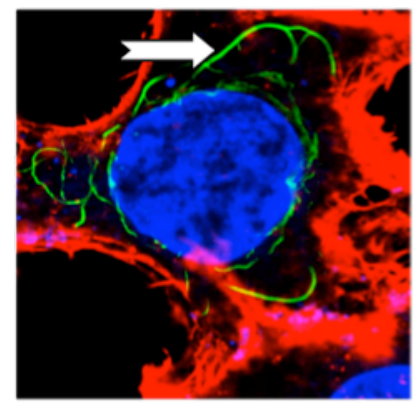

Schematic 4: Localization patterns of overexpressed full-length ASC and individual domains. Cells immunofluorescently stained for ASC (green), DNA (blue) and actin (red). Expression of full-length ASC (A) results in the formation of characteristic perinuclear aggregates termed 'specks'. However, both the CARD (B) and the PYD (C) are required in order for these structures to form, as expression of the individual domains results in the formation of previously characterized 'death filaments'.

The initial studies leading to the discovery of ASC provided evidence that it likely functioned in apoptotic pathways. The loss of ASC expression in breast cancer cells by methylation-induced gene silencing further implicated it as a clinically relevant tumor suppressor. Tumor suppressors are proteins, which present roadblocks in the pathway of cancer development. They often include proteins, which regulate cell cycle progression, particularly in the context of DNA damage, and those that are pro-apoptotic. In the course of tumor development, the genes, which encode these proteins are frequently silenced or mutated, which clears the way for unregulated proliferation combined with a decrease in cell death. Importantly, both alleles of a given tumor suppressor gene must be silenced in order for their loss of function to be complete. This is necessary because most loss of function mutations are recessive, and therefore one 
functioning allele would be able to generate sufficient protein to compensate for the loss of the second one. This requirement has become known as the two-hit hypothesis and was first proposed by Knudson in 1971 in the study of retinoblastoma (Knudson, 1971). Since the initial study in breast cancer, numerous studies have determined that loss of ASC expression is not limited to this type of cancer. Indeed, methylation-induced silencing of ASC has been a documented occurrence in multiple tumor types including ovarian (Akahira J, 2004,Terasawa, 2004), lung (Virmani, 2003), prostate (Partha M Das, 2006), glioblastoma (Stone, 2004), hepatocellular (Zhang C, 2007), colorectal (Yokoyama, 2003), and melanoma (Guan, 2003). Furthermore, methylation of ASC is associated with an increase in disease aggressiveness in both non-smallcell lung cancer (Zhang Z, 2006) and prostate cancer (Collard RL, 2006). These studies provide strong support for a functional role of ASC in tumor suppression.

While it has become well established that ASC functions as a proapoptotic protein, the precise mechanism still remains elusive. To date, several groups have demonstrated a role for ASC in multiple apoptotic pathways. Initial studies performed by the Vertino laboratory determined that ASC induced apoptosis in HEK 293 cells in a caspase-9 dependent manner. Using dominantnegative constructs, they showed that inhibition of caspase-9 prevented ASCmediated apoptosis. Additionally, they showed that the CARD domain of ASC was required (McConnell, 2000). However, studies performed in colon cancer cells revealed that ASC expression is induced through activation and binding of 
p53, and subsequently initiates apoptosis by mediating the translocation of the pro-apoptotic protein Bax from the cytoplasm to the mitochondria (Ohtsuka, 2004). Alternatively, studies in breast cancer suggest ASC may mediate apoptosis through cleavage and activation of caspase-8. While it does not appear that ASC is necessary for caspase-8 activation following the binding of death receptor ligands to their respective receptors, ectopic expression of ASC is sufficient to induce cleavage of caspase-8 resulting in apoptosis in the absence of any activation of the extrinsic pathway (Parsons MJ, 2006). Furthermore, it has also been demonstrated that ASC interacts directly with caspase- 8 via its PYD, and that ASC is unable to induce apoptosis in caspase-8 deficient MEFs (Masumoto, 2003).

Most recently, ASC has been implicated in the process of anoikis. Anoikis is the term applied to a specific type of programmed cell death that occurs when the interaction between a cell and its extracellular matrix is disrupted. This results in a loss of integrin-mediated survival signaling and induction of apoptosis (Frisch, 1994). In order for cancer cells to metastasize, they must invade into the basement membrane and surrounding tissues. This process requires them to survive in an anchorage-independent state, and therefore they must acquire mechanisms to prevent undergoing anoikis. Intriguingly, Parsons et al. has demonstrated that ASC is up-regulated in the non-transformed breast epithelial cell line MCF10A. Furthermore, siRNA-mediated knock-down of ASC resulted in a delay in detachment-induced cell death and inhibited the accumulation of the 
pro-apoptotic protein Bim (Parsons MJ, 2009). These results provide new insight into the advantages conferred upon tumor cells with the loss of this protein.

Although the original discovery of ASC occurred in the context of apoptosis studies, it was soon discovered that it also played a key role in inflammatory pathways. The first indication that ASC functioned in inflammatory pathways was the up-regulation of both the protein and mRNA in response to inflammatory stimuli including LPS, TNF-alpha, and IL-1 beta (Shiohara, 2002,Stehlik, 2003b). Subsequent studies suggested a role for ASC in the modulation of the activity of the transcription factor NF-kB.

$\mathrm{NF}-\mathrm{kB}$ is a central transcription factor in inflammatory processes as its activation results in transcription of a large number of pro-inflammatory genes including IL-1 beta as well as a number of pro-survival genes. Activation of NF$\mathrm{kB}$ is a highly regulated process. Under resting conditions, the active subunits of $\mathrm{NF}-\mathrm{kB}$ are sequestered in the cytoplasm by a protein called inhibitor of NF-kB (IkB). Activation and nuclear translocation of NF-kB occurs when the upstream regulatory IkB kinase (IKK), which consists of two related kinases (IKK alpha, and IKK beta) as well as a scaffolding unit (IKK gamma), phosphorylates IkB. This phosphorylation event results in the polyubiquitination and subsequent proteosomal degradation of $\mathrm{lkB}$. Upon the release, of $\mathrm{lkB}$, the active p50 and p65 subunits of NF-kB are able to enter the nucleus and initiate transcription (Karin, 2000,Perkins, 2004). 
Although several independent research groups have implicated ASC in the modulation of NF-kB activity, its precise role in this process has not been fully elucidated. Stehlik et al. demonstrated that overexpression of ASC with the PYD-containing proteins NALP3 and Pyrin in HEK 293 cells resulted in the activation of NF-kB. In contrast, the same study demonstrated that overexpression of ASC as well as other PYD-containing proteins caused an inhibition in the activation of NF-kB in response to the inflammatory stimulus TNF-alpha. This inhibition was overcome upon the co-expression of ASC once again with NLRP3 and Pyrin. Furthermore, an increase in the degradation of IkB-alpha was observed upon siRNA-mediated suppression of ASC.

Furthermore, this group also showed that ASC inhibited NF-kB activation through an interaction with the IKK complex mediated by its PYD. These results indicate that ASC exerts a PYD-dependent inhibitory effect on NF-kB activation, which can be overcome upon its interaction with either NLRP3 or Pyrin (Stehlik, 2002). Whether NLRP3 or Pyrin impact NF-kB activation by disrupting the interaction between ASC and the IKK complex, or whether they utilize ASC to stimulated IKK activity has yet to be determined.

Other studies have also demonstrated a role for ASC in the activation of NF-kB upon its co-expression with another NLR family member known as NLRC4 (Ipaf/CARD12/CLAN). NLRC4 is structurally similar to NLRP3 with the exception that it possesses a CARD domain as its protein-interaction domain rather than a PYD, and senses cytosolic flagellin (Miao EA, 2006). Masumoto et al 
demonstrated that the interaction between ASC and NLRC4 resulted in an increase in NF-kB activation that was enhanced upon deletion of the LRR region of NLRC4. Furthermore, they also confirmed that the increase in NF-kB activity was mediated by the PYD of ASC (Masumoto, 2003). Another group expanded on these results and demonstrated that caspase-8 was necessary for NLRC4 and ASC-mediated NF-kB activation, and that this activation could be inhibited by the by FLIP, a cellular inhibitor of caspase-8 activation (Hasegawa, 2005). Evaluation of the role of ASC in cytokine production in response to Porphyromonas gingivalis also provided support for the hypothesis that ASC actively contributes to NF-kB activation. The generation of THP-1 monocytic cells with a stable knock-down of ASC demonstrated that NF-kB activation is delayed and there is a decrease in p65/p50 DNA binding in the absence of ASC. Furthermore, loss of ASC resulted in a decrease in IL-6, IL-8, and IL-10 in response to $P$. gingivalis as well as several different TLR agonists (Taxman, 2006).

Further evaluation of the role of ASC in inflammatory processes determined that it directly interacts with pro-caspase- 1 , which is responsible for converting pro-IL-1-beta into its active form. This activation occurs via proteolytic cleavage at the key aspartic acid residue. The interaction between ASC and caspase- 1 is mediated by a homotypic interaction between the CARD domain of ASC and the CARD domain of caspase-1. In addition, this interaction results in the processing of caspase- 1 into its active form, as well as downstream processing and release of IL-1 beta and forced oligomerization of the ASC CARD 
domain is sufficient to cause caspase-1 activation (Srinivasula, 2002,Stehlik, 2003b). Furthermore, it was demonstrated that ASC co-localizes with procaspase-1 and recruits it into the characteristic perinuclear aggregates upon overexpression (Stehlik, 2003b).

The importance of ASC in caspase-1 activation was solidified with the generation of the knock-out mouse. Morphologically, there are no deficits observed in these mice in spite of the demonstrated role of ASC in apoptosis. However, in response to LPS, they are completely impaired in their ability to generate active caspase-1 and secrete the pro-inflammatory cytokines processed by caspase-1, IL-1 beta and IL-18. Furthermore, loss of ASC had no impact on the increased production of pro-IL-1 beta. Therefore, the role of ASC in IL-1 beta production is limited to processing the precursor into its active form, rather than on its transcriptional upregulation. Intriguingly, there was no deficit in these mice in the production of other inflammatory cytokines such as TNF-alpha and IL-6. Production of these two cytokines is regulated by the transcription factor NF-kB. Therefore, in mice, loss of ASC does not impair NF-kB activation (Yamamoto, 2004).

Caspase- 1 is the first identified a member of a family of cysteine proteases, which have well-established roles in inflammation as well as apoptosis. It was initially discovered as the key enzyme required for the generation of 17.5 $\mathrm{kD}$ mature IL-1 beta from the inactive precursor and thus was termed IL-1 converting enzyme (ICE) (Thornberry, 1992). In addition, activation of caspase-1 
occurs in both monocytic and endothelial cells in response to the same pathogenic stimuli that initiate IL-1beta secretion such as LPS (Schumann, 1998). Caspase-1 cleaves pro-IL-1 beta at aspartic acid-alanine residues at positions 116-117 and cleaves IL-18 at aspartic acid-tyrosine residues at positions 35-36. Interestingly, caspase-1 is not responsible for the generation of mature IL-1alpha, in spite of the similarities observed regarding the bioactivity of these two members of the IL-1 family (Howard AD, 1991). Consistently, gene ablation studies with caspase-1 have confirmed its necessity for the maturation of IL-1 beta, as mice deficient in this protein are severely impaired in their ability to secrete the processed cytokine upon pathogenic challenge ( $\mathrm{Li}, 1995)$.

While caspase-1-mediated conversion of pro-IL-1beta to the mature form occurs in the cytoplasm (Brough D, 2007), other subcellular localization patterns for caspase-1 have been observed. First, caspase- 1 has been found to redistribute from the cytoplasm to the nucleus in response to treatment with TNFalpha. Furthermore, this nuclear localization is mediated by the pro-domain of caspase- 1 as demonstrated by the fact that both the full-length protein and the individual pro-domain exhibit a nuclear and cytosolic localization pattern, while the p10 and p20 domains are restricted to the cytoplasm (Mao, 1998). Interestingly, elevated expression of caspase- 1 and its nuclear localization are associated with a favorable outcome in neuroblastoma patients, as a strong correlation exists between caspase-1 expression and patient survival (Nakagawara A, 1997). 
Recently, a novel mechanism for regulating caspase-1 activation has emerged. Levin et al have demonstrated that thermal stress can inhibit caspase-1 activation by both the NLRP1 and NLRP3 inflammasomes. In addition, they determined that heat shock caused the sequestration of caspase-1 into an insoluble fraction, which may indicate its incorporation into a large inhibitory protein complex (Levin TC, 2008). Considering both caspase-1 substrates IL1 beta and IL-18 are potent endogenous pyrogens, the increase in body temperature caused by these cytokines may function as an important negative feedback mechanism to prevent their excessive release.

Like ASC, caspase-1 has also been implicated in NF-kB activation. Unlike maturation of IL-1 beta, this activation is independent of caspse- 1 enzymatic activity as the enzymatically inactive mutant retains the ability to activate NF-kB. In addition, the CARD domain, which is only present in pro-caspase-1 is both necessary and sufficient for this function. One mechanism by which caspase-1 achieves NF-kB is through binding to RIP2 via CARD-CARD interaction (Lamkanfi M, 2004).

Interestingly, one mechanism by which ASC may modulate NF-kB activation is by exerting influence on the interaction between caspase-1 and RIP2. While at low levels, ASC can stimulate NF-kB activation; at higher expression levels ASC disrupts the interaction between caspase-1 and RIP2, which results in a decrease in NF-kB activation, but an elevation in IL-1beta secretion. Therefore, ASC may compete with RIP2 for caspase-1 binding in order to engage it in 
inflammasome-mediated IL-1beta activation and down-regulate NF-kB activation. In monocytes, the pathway, which is favored by caspase-1, may be time dependent. At early time-points, activation of NF-kB would be necessary in order to generate the IL-1beta precursor. However, at later times caspase-1 would be redirected from NF-kB activation to the task of IL-1beta processing (Sarkar, 2006).

Like ASC, the functional role of caspase-1 does not appear to be strictly limited to inflammatory processes. Recent evidence has accumulated in favor of caspase-1 serving as the primary mediator of an inflammatory form of apoptosis, which has been termed pyroptosis. While other members of the caspase family of proteases have long been established to be key players in both the intrinsic and extrinsic apoptosis pathways, gene ablation studies with caspase- 1 have found no functional deficit in response to normal apoptotic stimuli. The process of pyroptosis is primarily distinguished from apoptosis by its inflammatory nature. The process of apoptosis causes characteristic morphological changes that include DNA condensation and the dissociation of the cell into membranecontained fragments. These fragments can then be phagocytosed and their contents recycled. One key hallmark of this process is that no inflammation or tissue disruption occurs because all of the intracellular contents remain contained within the membrane fragments. Pyroptosis, on the other hand, is characterized by loss of cell membrane integrity and the release of intracellular contents, which initiate a pro-inflammatory response. Furthermore, it is dependent upon caspase1, rather than the classical apoptotic caspases. This type of cell death was 
initially observed and characterized in response to infection with intracellular bacteria Salmonella and Shigella. However, subsequent studies have found that it can also occur in response to extracellular bacterial ligands such as LPS (Bergsbaken T, 2009,Fink SL, 2005,Fink SL, 2006).

While the role of caspase- 1 in mediating pyroptotic cell death is widely accepted, there is some controversy regarding the necessity of ASC for this process. Recently, the Alnemri lab proposed the formation of the pyroptosome, a supramolecular assembly, similar to the inflammasome, in which ASC oligomers serve as a platform for caspase-1 activation. By generating THP-1 cells, which stably express GFP-ASC at endogenous levels, theses researchers were able to observe the formation of small, perinuclear 'specks' following treatment with bacterial ligands such as LPS. Furthermore, the formation of these structures preceded cell death, which possessed the morphological characteristics of pyroptosis by only a short time as determined by live-cell imaging ( $T$ FernandesAlnemri, 2007). However, studies performed by another group with the intracellular bacterium Shigella flexneri, found that while caspase-1 and NLRC4 were essential to Shigella-induced pyroptosis, ASC was not required. Upon infection of macrophages derived from caspase-1 or NLRC4 deficient mice, this group found that the release of the pyroptosis marker LDH was delayed.

However, no such change in kinetics was observed in macrophages derived from ASC-null mice. These results suggest that ASC is dispensable for pyroptotic cell death. One explanation for this result offered by the researchers is that ASC may 
promote survival of infected macrophages in the absence of caspase-1 or NLRC4. This possibility would coincide with previous reports establishing ASC as an activator of NF-kB (Suzuki T, 2007).

\section{Regulation of the Inflammasome}

Regulation of inflammasome formation and activation is an ongoing area of research. Currently, the primary regulatory mechanism that has been identified is the existence of small, single-domain proteins, which function in a dominantnegative capacity and thereby disrupt key protein interactions within the inflammasome complex. These regulatory proteins can be divided into two distinct categories, based upon the area they target within the activation platform. Those consisting of an individual PYRIN domain function by disrupting the interaction between the NLR receptor and the key adaptor protein ASC, and are frequently referred to as PYRIN-only-proteins or POPs. The regulatory proteins in the second category are composed of an individual CARD domain, which is utilized to interfere with the recruitment of caspase-1 to the central adaptor ASC. These proteins are broadly termed CARD-only-proteins or COPs (Stehlik C, 2007).

The first PYRIN-only protein to be characterized was termed POP1. The gene encoding this protein is found on chromosome $16 \mathrm{p} 12.1$, which is the same area where the gene for ASC is found. Furthermore, the amino acid sequence of POP1 was determined to be $64 \%$ identical with the PYD of ASC and 
immunostaining revealed that while POP1 localized diffusely to the nucleus and cytoplasm of the cell, it was recruited into the characteristic 'speck' structures when co-expressed with ASC. Functionally, POP1 exhibits the ability to modulate activation of ASC-mediated inflammatory pathways including NF-kB activation and IL-1 beta secretion (Stehlik, 2003a). In addition, further studies have revealed the binding region of the ASC, where it interacts with POP1. POP1 interacts with a negatively charged region of ASC located on the first and fourth helices, which includes aspartic acid residues at residues 6,13 , and 48 , and a glutamic acid residue at residue 13 (Srimathi T, 2008).

Recently, our laboratory in addition to others identified and described an additional cellular pyrin only protein, cPOP2. We demonstrated that this protein interfered with caspase-1-mediated IL-1 beta processing. This inhibition is most likely achieved via direct interaction between CPOP2 and ASC as well as CPOP2 and the NLRP receptor PAN1. Other potential binding partners for cPOP2 include PAN2, NLRP12, and NLRP1 as demonstrated by yeast-2 hybrid assay (Dorfleutner, 2007a). In addition, cPOP2 has also been found to hinder NFkappa B activation in response to both TNF-alpha stimulation and overexpression of p65. Furthermore, expression of cPOP2 decreased nuclear accumulation of p65 (Bedoya F, 2007). Analysis of the subcellular localization of CPOP2 revealed that its localization was very similar to that of POP1 in that it localized diffusely throughout the cell when expressed alone and was recruited 
into the perinuclear aggregate 'speck' structures upon co-expression with ASC (Bedoya F, 2007,Dorfleutner, 2007a).

In addition to the cellular POPs, genomic studies of several viruses have yielded the discovery of viral counterparts to these regulators of inflammasome activation. These proteins likely evolved as a mechanism to aid the viruses in their evasion of the host immune system by preventing the maturation and secretion of IL-1beta. The initial discovery of a viral protein capable of modulating inflammasome function was made in the rabbit Myoxma virus. This viral POP is located on the M13L gene of the virus. The product of this gene was determined to block casapse-1 processing and subsequent IL-1 beta and IL-18 processing and secretion. This inhibition is most likely achieved via direct interaction with the adaptor protein ASC as it has been demonstrated to both colocalize and directly bind to ASC via PYD-PYD interaction (Dorfleutner, 2007b,Johnston JB, 2005). Furthermore, deletion of this protein from the virus resulted in decreased viral dissemination and prevented viral replication in lymphocytes and peripheral blood mononuclear cells. Loss of this protein also resulted in more rapid disease resolution underlining its importance in maintaining the virulence of this virus (Johnston JB, 2005)

In addition to the Myxoma virus, another Poxvirus, the Shope Fibroma virus (SFV), also encodes a POP. Upon transfection, this protein localizes diffusely throughout the nucleus and cytoplasm at forty-eight hours followed by the formation of punctate structures at seventy-two hours. In addition, upon co- 
expression with the inflammasome adaptor protein ASC, the SFV POP is recruited to the characteristic perinuclear 'speck'. These localization patterns are reminiscent of those of the Myxoma viral POP as well as cPOP1. Functionally, the SFV POP is also capable of inhibiting IL-1 beta secretion, and both the SFV and Myxoma POPs activate NF-kB. While this activation may initially appear counterintuitive due to the numerous anti-inflammatory genes under the regulation of NF-kappa B, this transcription factor is very commonly activated by a number of viruses, possibly as a mechanism to prevent virally mediated apoptosis in host cells (Dorfleutner, 2006).

The second group of inflammasome regulatory proteins, the CARD-only proteins (COPs) function in a manner similar to that of the Pyrin-only proteins. Like the POPs, the COPs also serve to inhibit the maturation and secretion of IL1 beta. However, they achieve this via binding to pro-caspase-1 via CARDCARD interaction and thereby preventing its autocatalytic processing and activation. Several mammalian COPs have been identified including ICEBERG, Pseudo-ICE, COP, and INCA. ICEBERG, COP, and INCA all map to chromosome $11 \mathrm{q} 22$, which is the same region where the Pro-caspase- 1 gene is located along with the genes for caspases 4 , and 5 , which also function in inflammatory pathways. Therefore, COP proteins are generally believed to have arisen from gene duplication.

All of the CARD-only proteins exhibit high sequence homology with the CARD domain of caspase-1, and are generally expressed in the same tissues as 
caspase-1. Furthermore, they have each been demonstrated to bind to the CARD-containing pro-domain of caspase- 1 and this interaction has been correlated with a decrease in the autoprocessing of caspase-1 as well as inhibition of IL-1 beta secretion. Interestingly, in addition to their ability to interact with caspase-1, many of the CARD-only proteins also exhibit the ability to selfassociate as well as bind to one another.

The first CARD-only protein to be identified and characterized was ICEBERG. ICEBERG is primarily expressed in cardiac and placental tissues, and is $52 \%$ identical to the CARD of caspase- 1 . It is transcriptionally upregulated in response to stimulation with the inflammatory cytokine TNF-alpha, as well as with the bacterial component lipopolysaccharide (LPS) in monocytic and epithelial cells (Druilhe, 2001,Humke, 2000). Pseudo-ICE is 97\% identical to the pro-domain of caspase-1, and exhibits a near identical tissue expression profile. Therefore, it is postulated that these two proteins may be under the same transcriptional regulation. In addition to its interaction with caspase-1 and other CARD-only proteins, Pseudo-ICE has the capability of interacting with the caspase-1 activating protein RIP2. Furthermore, Pseudo-ICE is unique among the COPs in its ability to also function as a potent activator of NF-kB (Druilhe, 2001). COP exhibits $97 \%$ identity with the CARD domain of caspase- 1 , and is also capable of interacting with RIP2 in addition to caspase-1 (Lee, 2001). INCA, the most recently discovered CARD only protein, is $81 \%$ identical to the CARD 
domain of caspae-1, and is upregulated in response to IFN-gamma, but not LPS or TNF-alpha (Lamkanfi, 2004).

In addition to the CARD-only proteins, caspase-12 has also been identified as a modulator of IL-1 beta secretion. Caspase-12 gene ablation studies have demonstrated a resistance to sepsis and an increase in the capacity of the immune system to clear pathogenic bacteria. Furthermore, caspase-12 is able to bind to caspase- 1 and prevent its activation, and by extension the downstream processing of IL-1 beta independent of its enzymatic activity (Saleh $M, 2006)$. This data suggests that caspase-12 may also function as a dominant negative inhibitor of inflammasome activation.

In addition to the PYRIN-only proteins and the CARD only proteins, there is an additional modulator of inflammasome activity called Pyrin. Pyrin is the product of the MEFV gene, which is located on chromosome 16 and was identified as the cause of the most common autoinflammatory disorder known as Familial Mediterranean Fever (FMF) (Bernot A, 1997). FMF is a genetically recessive disease characterized by recurrent, self-limiting fevers, which are of short duration. It is predominantly seen in four populations: non-Ashkenazi Jews, Turks, Armenians, and Arabs. Several physical and environmental stimuli have been identified as triggers for the recurrent fevers including, emotional stress, high-fat meals, infections, menstrual cycle, cold temperatures, and some drugs. Clinical manifestations include a fever, which lasts for 12-72 hours, and then resolves spontaneously. In addition, involvement of the joints, abdomen, chest, 
skin, and kidneys may also be observed. The most severe clinical complication is Type AA amyloidosis, which is usually observed in the kidneys in the form of chronic nephrotic syndrome. Currently, colchicine is the primary choice for treatment (Fonnesu C, 2008).

Structurally, Pyrin is 781 amino acids long, and consists of multiple domains. It has an N-terminal Pyrin domain, a zinc finger domain, a Coiled Coil, domain and a B30.2 domain at its C-terminus. Interestingly, it is the B30.2 domain where the majority of the mutations associated with FMF occur. Functionally, it is believed that Pyrin as an inhibitor of inflammasome activation based upon the phenotype of FMF patients. Initial studies with Pyrin demonstrated that it could interact and modulate the functions of ASC. However, since then, studies have shown that it can also interact with other inflammasome components including NLRP3 and caspase-1. As a result, inflammasome modulation by Pyrin is complex and multi-faceted.

The interaction between Pyrin and ASC was first established by the Gumucio laboratory. Using a yeast two-hybrid screen they identified ASC as an interaction partner for Pyrin. Further analysis demonstrated that while Pyrin localized diffusely in the cytoplasm when overexpressed alone, upon coexpression with ASC, it was recruited to the characteristic ASC 'specks'. This interaction was found to be mediated by the Pyrins N-terminal PYD. Functionally, this group first explored the role of Pyrin in ASC-mediated 
apoptosis. They discovered that co-expression of Pyrin with ASC attenuated ASC-mediated apoptosis (Richards, 2001).

With regard to its impact on inflammasome function, it has been demonstrated that Pyrin exhibits both inhibitory and activating properties. The inhibitory capacity of Pyrin was first demonstrated by Chae et al, through the generation of mice, which expressed a truncated form of Pyrin lacking the B30.2 domain. Upon treatment with lipopolysaccharide, these mice exhibited elevated fever and increased lethality over control. In accordance with this, macrophages from these mice exhibited elevated levels of caspase-1 activation and IL-1 beta secretion following stimulation with LPS. Furthermore, this group determined that full-length Pyrin competes with caspase-1 for binding with ASC, which results in a decrease in caspase-1 activation (Chae, 2003). Interestingly, subsequent studies demonstrated that a direct interaction between Pyrin and caspase-1 occurs independent of ASC. Specifically, the B30.2 domain of Pyrin was shown to interact with catalytic p10 and p20 subunits of caspase-1. In addition, knock-down of Pyrin in monocytic cells resulted in an increase in IL1 beta secretion in response to pathogenic challenge, whereas overexpression of the B30.2 domain resulted in an inhibition of IL-1beta, which further indicates a suppressive role for Pyrin in inflammasome activation (Chae, 2006,Papin S, 2007). Conversely, other research groups have shown Pyrin to function as an inflammasome activator. First, an increase in IL-1 beta secretion was observed in response to increasing concentrations of Pyrin in a reconstituted 
inflammasome model (Stehlik, 2003b). Intriguingly, this increase was independent of ASC. Furthermore, knock-down of Pyrin in both the monocytic cell line and PBMCs resulted in a decrease in IL-1 beta secretion in response to LPS (Seshadri S, 2007).

Intriguingly, not only does Pyrin's interaction with caspase-1 influence inflammasome activation, but caspase-1 can impact Pyrin's functions as well. It has been recently demonstrated that Pyrin not only binds to caspase-1, but it is also a substrate and is cleaved at aspartic acid residue 330 . The $\mathrm{N}$-terminal cleavage product of Pyrin generated by caspase-1 cleavage subsequently activates NF-kB. The N330 cleavage product achieves this by mediating the translocation of the p65 subunit to the nucleus as well as causing calpainmediated degradation of IkB-alpha (Chae JJ, 2008).

In the past year, a role for Pyrin in other cellular processes has emerged. First, Pyrin was found to interact with the pro-apoptotic protein Siva. Siva is a p53 target gene that can modulate apoptosis in response to chemotherapy. It is also capable of interacting with anti-apoptotic Bcl-2 family members and the cytoplasmic tail of several TNF receptors. The B30.2 domain of Pyrin and exon 1 of Siva are required in order for these proteins to interact. Intriguingly, Pyrin can recruit Siva to ASC 'specks' when all three proteins are expressed. Therefore, Pyrin may function as a bridging protein that enables cross-talk between the apoptotic pathways of ASC and Siva (Balci-Peynircioglu B, 2008). In addition, new evidence has unveiled a potential role for both ASC and Pyrin in the process 
of cell motility. Waite et al demonstrated that both Pyrin and ASC can localize to the leading edge of motile cells, which was unaffected by FMF-associated mutations. Furthermore, they both co-localized in areas rich in polymerizing actin generated by the bacterium Listeria, known as 'rocket tails'. While ASC was unable to co-localize with any of the proteins necessary for rocket tail formation, Pyrin was determined to directly interact with both VASP and Arp3. Furthermore, both of these proteins could be recruited to ASC specks upon overexpression with Pyrin (Waite AL, 2009).

The association between inflammation and cancer has been long established. Many tumors arise in patients suffering from chronic inflammation, and the inflammatory component of the tumor microenvironment is essential for the processes of tumor progression and invasion. IL-1beta is a key proinflammatory cytokine, which upon its release, can initiate a host of systemic and local responses. Current literature has established inflammasome formation as crucial step in the activation and secretion of IL-1beta, and the adaptor protein ASC is necessary for its assembly. However, the regulatory mechanisms safeguarding the integrity of this process have not been fully elucidated. Therefore, in order to gain a better understanding of how ASC modifications can impact IL1 beta maturation, these studies will aim to identify novel regulatory mechanisms that define the circumstances under which ASC participates in inflammasome complex formation. Overall, this work will aim to provide further insight into the intricate cellular processes involved in inflammation and their impact on disease. 


\section{Study 1:}

Activation of Inflammasomes Requires Intracellular Redistribution of the Apoptotic Speck-like Protein Containing a Caspase Recruitment Domain (ASC) 


\section{ABSTRACT}

Activation of caspase- 1 is essential for the maturation and release of interleukin (IL)-1 $\beta$ and IL-18, and occurs in multi-protein complexes, referred to as inflammasomes. The apoptosis-associated speck-like protein containing a caspase recruitment domain (ASC) is the essential adaptor protein for recruiting pro-caspase-1 into inflammasomes, and consistently gene ablation of ASC abolishes caspase- 1 activation and secretion of IL-1 $\beta$ and IL-18. However, distribution of endogenous ASC has not yet been examined in detail. In the present study we demonstrated that ASC localized primarily to the nucleus in resting human monocytes macrophages. Upon pathogen infection ASC rapidly redistributed to the cytosol, followed by assembly of perinuclear aggregates, containing several inflammasome components, including caspase-1 and Nod-like receptors (NLRs). Prevention of ASC cytosolic redistribution completely abolished pathogen induced inflammasome activity, which affirmed that cytosolic localization of ASC is essential for inflammasome function. Thus, our study characterized a novel mechanism of inflammasome regulation in host defense. 


\section{INTRODUCTION}

Inflammatory reactions in response to pathogen infection are highly coordinated processes. Leukocytes are recruited to sites of infection where they become activated and enlist tissue cells to aid with pathogen clearance. This response is orchestrated by a complex array of soluble mediators, including locally released cytokines and chemokines, such as macrophage-released interleukin (IL)-1 $\beta$ and IL-18. Generation of mature IL-1 $\beta$ and IL-18 is regulated at multiple steps, including transcription, posttranslational processing and receptor binding [Dinarello 1998]. Processing into the bioactive secreted 17 or $18 \mathrm{kDa}$ forms, is dependent on the proteolytic activity of caspase- 1 , which itself becomes activated in molecular platforms, referred to as inflammasomes [Martinon 2002]. Inflammasomes emerged recently as multi protein complexes that link recognition of damage-associated molecular patterns (DAMPs) by members of the Nod-like receptor (NLR) ${ }^{3}$ family of cytosolic pattern recognition receptors (PRRs) to the activation of caspase-1 and processing and release of the pro-inflammatory cytokines IL-1 $\beta$ and IL-18 [Franchi 2006,Mariathasan 2007,Martinon 2007,Ogura 2006,Stehlik 2007a,Ting 2006]. Core inflammasome proteins therefore include, NLRs, the pyrin domain (PYD) and caspase recruitment domain (CARD)-containing adaptor protein apoptotic speck-like protein containing a CARD (ASC), and caspase-1 [Agostini 2004,Martinon 2002]. The inflammasome-initiating event is recognition of intracellular DAMPs derived 
from pathogens (PAMPs) or host (danger or stress signals) by a cytosolic NLR. Thus, inflammasomes are assumed to form in the cytosol of phagocytic cells, such as monocytes and macrophages. NLRs undergo ATP-dependent oligomerization in response to DAMP recognition, and recruit ASC by PYD-PYD interaction [Duncan 2007,Faustin 2007]. Subsequently, caspase-1 is recruited through the CARD of ASC, which is essential for its activation [Srinivasula 2002,Stehlik 2003]. Consistently, macrophages deficient in ASC are impaired in their ability to activate caspase- 1 in response to infection and tissue damage, emphasizing its central role for the activation of inflammatory caspases [Mariathasan 2004,Yamamoto 2004].

NLRP3 (Cryopyrin) is activated by Gram positive bacteria and multiple DAMPs including MDP, bacterial and viral RNA, toxins that cause a decrease in intracellular potassium levels, genomic DNA, irritants, such as trinitrochlorobenzene and dinitro-1-fluorobenzene and reactive oxygen species, as well as uric acid and calcium pyrophosphate crystals [Dostert 2008,Kanneganti 2006b,Mariathasan 2006,Martinon 2004,Muruve 2008,Sutterwala 2006,Watanabe 2007]. NLRP3 also functions in concert with P2X7 receptors recognizing extracellular ATP [Kanneganti 2006b,Mariathasan 2006]. Due to the central role of IL-1 $\beta$ and IL-18 in regulating inflammation and immunity, dysregulation of pathways leading to caspase-1 activation and the resulting uncontrolled secretion of these pro-inflammatory mediators is directly linked to human inflammatory disorders. Variants of NLRP1 are associated with 
autoimmune diseases that cluster with vitiligo [Jin 2007]. NLRP3-containing inflammasomes were recently linked to contact hypersensitivity, sunburn, essential hypertension, gout and pseudogout, and elevated expression of NLRP3 is detected in synovial fluids of RA patients [Feldmeyer 2007,Martinon 2006,Omi 2006, Rosengren 2005, Watanabe 2007]. Furthermore, hereditary mutations in NLRP3 rendering the protein constitutively active, are directly linked to cryopyrinassociated periodic syndromes (CAPS) [Church 2006,Stojanov 2005]. Mutant NLRP3 proteins efficiently form complexes with ASC to mediate caspase-1 activation independent of an activating ligand. This finding demonstrates the potential benefits of controlling the accessibility of ASC for cytosolic NLRs [Stehlik 2007b]. Several mechanisms have been proposed by which activation of inflammasomes might be regulated, including single PYD or CARD-containing proteins and pyrin [Stehlik 2007b]. However, regulation of inflammasomes still remains largely elusive.

Here we describe a novel regulatory mechanism for inflammasome assembly. We propose that ASC is sequestered in the nucleus in resting monocytes and macrophages, and only becomes available to bridge NLRs and caspase-1 in the cytosol, once macrophages are activated in response to pathogen infection and cellular stress. Such a mechanism might provide an additional safety checkpoint to limit spontaneous activation of caspase-1 in resting cells. 


\section{EXPERIMENTAL PROCEDURES}

\section{Cell culture.}

THP-1, U-937, HL-60, and HEK293 cells were obtained from the American Type Culture Collection and maintained as recommended by ATCC. HEK293 cells were transiently transfected using Polyfect (Qiagen) according to the manufacturer's instructions. THP-1 cells were used at low passage numbers and were regularly tested for mycoplasma infection (MycoAlert, Lonza). THP-1 cells were transiently transfected using the Nucleofector II (Amaxa) with solution V and protocol V001 using two ASC-specific siRNAs targeting the 3'UTR and nucleotides 474-492 of the ASC ORF [Stehlik 2002]. THP-1 cells were seeded into collagen I $\left(5 \mu \mathrm{g} / \mathrm{cm}^{2}\right)$-coated glass cover slips and differentiated into adherent macrophages by overnight culture in medium supplemented with 15 $\mathrm{ng} / \mathrm{ml}$ of phorbol 12-myristate 13-acetate (PMA) and further cultured for 2 days. Where indicated, cells were treated with $2 \mu \mathrm{g} / \mathrm{ml}$ of $E$. coli total RNA (Ambion), or $2 \times 10^{5} \mathrm{cfu} / \mathrm{ml}$ of heat killed Legionella pneumophila (HKL) or Staphylococcus aureus (HKSA) (InvivoGen).

\section{Isolation of primary monocytes from human peripheral blood.}

Human monocytes were isolated by Ficoll-Hypaque centrifugation (Sigma) from heparanized blood. Mononuclear cells were removed, washed and resuspended 
in serum-free DMEM and isolated by adherence to plastic dishes. Adherent monocytes were washed, incubated in complete RPMI 1640 media overnight in glass chamber slide, and were left untreated or treated with $2 \mu \mathrm{g} / \mathrm{ml}$ of $E$. coli total RNA (Ambion). Macrophages were obtained by culture of adherent monocytes on collagen type I $\left(5 \mu \mathrm{g} / \mathrm{cm}^{2}\right)$-coated glass cover slips for 7 days in medium containing $20 \%$ FBS. Alternatively, monocytes were isolated from PBMCs by countercurrent centrifugal elutriation in the presence of $10 \mu \mathrm{g} / \mathrm{ml}$ polymyxin B sulfate using a JE-6B rotor (Beckman Coulter).

\section{Expression plasmids.}

The nuclear localization sequence (NLS) from the SV40 large T antigen (DPKKKRKV) was utilized to generate a 3xNLS-ASC fusion protein with RFP epitope tags in pcDNA3 (Invitrogen) and pMSCV-puro (Clontech) expression vectors [Lanford 1986]. 3 silent point mutations were introduced in the siRNA recognition sequence (Quick-change, Stratagene) to prevent siRNA-mediated degradation of ASC and NLS-ASC. Authenticity of all plasmids was confirmed by sequencing. All other plasmids have been previously described [Dorfleutner 2007a].

\section{Retroviral infections.}

293GP2 cells (Clontech) were transiently transfected with RFP-tagged ASC, 
NLS-ASC, empty pMSCV-puro, or GFP-ASC in modified pMSCV-puro expression vectors (Clontech) or pRNATin-H1.4 (Genscript), in combination with a VSV-G-encoding expression plasmid to produce a pseudo-typed recombinant retrovirus. $5 \times 10^{5}$ THP-1 cells were infected by spinoculation in the presence of $6 \mu \mathrm{g} / \mathrm{ml}$ polybrene (Sigma) at $32^{\circ} \mathrm{C}$. Stable cells were selected 72 hours post infection with $3 \mu \mathrm{g} / \mathrm{ml}$ puromycin or $400 \mu \mathrm{g} / \mathrm{ml}$ hygromycin for 14 days. Expression of ASC and NLS-ASC was verified in pooled cell populations by immunoblot, and knock-down of ASC was verified by immunoblot in cells sorted by FACS for cGFP expression.

\section{Generation of shRNA plasmids.}

shRNA expression constructs were generated by inserting double stranded oligonucleotides into the Mlu/ and Xho/ sites of pRNATin-H1.4 (Genscript) downstream of the RNA polymerase III H1 promoter. Stable knock down of ASC in THP-1 cells was achieved by infection with a VSV-G-pseudotyped retrovirus encoding an shRNA targeting ASC (targeting sequence gctcttcagtttcacacca) or firefly luciferase (targeting sequence gatttcgagtcgtcttaat) as control by spinoculation on three consecutive days. Cells were selected in hygromycin (400 $\mu \mathrm{g} / \mathrm{ml}$ ) for 14 days and sorted by FACS for cGFP expression, which is encoded in the vector backbone independent from the shRNA.

Immunofluorescence microscopy. 
Adherent cells on collagen I-coated cover slips $\left(5 \mu \mathrm{g} / \mathrm{cm}^{2}\right)$ were fixed in $3.7 \%$ paraformaldehyde, incubated in $50 \mathrm{mM}$ glycine for 5 minutes and permeabilized and blocked with $0.5 \%$ saponin, $1.5 \%$ BSA, $1.5 \%$ normal goat serum for 30 minutes. ASC was immunostained with affinity purified monoclonal anti-ASC antibodies (MBL, $1 \mu \mathrm{g} / \mathrm{ml}$ ) directed to the PYD, affinity purified polyclonal antibodies directed to the CARD from Chemicon, Calbiochem, and Apotech (1:200), custom raised affinity purified polyclonal antibodies directed to the linker (CS3; 1: 2,500), and monoclonal anti-myc antibodies (Santa Cruz Biotechnology, 1:350). Caspase-1 was immunostained with a monoclonal anti-caspase-1 antibody from BD Biosciences (1:500) and a polyclonal antibody from Santa Cruz Biotechnology (1:50); NLRP3 was immunostained with monoclonal anti-NLRP3 antibodies from Abcam (1:50) and Santa Cruz Biotechnology (1:50); and IL-1 $\beta$ with a polyclonal antibody from Santa Cruz Biotechnology (1:50). Secondary Alexa Fluor 488, 546, and 647-conjugated antibodies, ToPro-3, DAPI, and phalloidin were from Molecular Probes. Cells were washed with PBS containing 0.5\% saponin, and cover slips were mounted using Fluoromount-G (Southern Biotech). Suspension cells were fixed and stained as above and adhered to poly-L-lysine-coated slides using a cytocentrifuge (StatSpin). Images were acquired by confocal laser scanning microscopy on a Zeiss LSM 510 or LSM 510 Meta, and epifluorescence microscopy on a Nikon TE2000E2 with a 100x oil immersion objective and image deconvolution. 


\section{Subcellular fractionation.}

$10^{6}$ cells were resuspended in hypotonic lysis buffer (10 mM Tris-HCL pH 7.4, 10 $\mathrm{mM} \mathrm{NaCl}, 3 \mathrm{mM} \mathrm{MgCl} 2,1 \mathrm{mM}$ EDTA, and $1 \mathrm{mM}$ EGTA, supplemented with protease and phosphatase inhibitors), incubated on ice, adjusted to $250 \mathrm{mM}$ sucrose, and lysed in a Dounce homogenizer. Samples were initially centrifuged at $4^{\circ} \mathrm{C}$ at $1,000 \mathrm{xg}$ for 3 minutes to remove any intact cells and then centrifuged at $4^{\circ} \mathrm{C}$ at $2,000 \mathrm{xg}$ for 10 minutes to pellet the nuclei. The cytosolic supernatant was removed, and the nuclear pellet was then washed three times in hyptonic lysis buffer with the addition of $250 \mathrm{mM}$ sucrose and $0.1 \% \mathrm{NP}-40$ and incubated

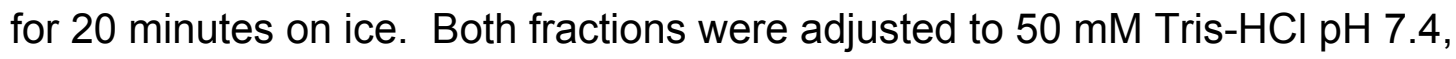
$20 \mathrm{mM} \mathrm{NaCl}, 3 \mathrm{mM} \mathrm{MgCl} 2,250 \mathrm{mM}$ sucrose, $0.5 \%$ deoxycholate, $0.1 \%$ SDS, $0.2 \% \mathrm{NP}-40$, and protease and phosphatase inhibitors, and fully solubilized by brief sonication. $50 \mu \mathrm{g}$ of protein lysates were separated by SDS-PAGE, transferred to a PVDF membrane, and probed with anti-ASC antibodies (CS3) and HRP-conjugated secondary antibodies (Amersham Pharmacia) in conjunction with an ECL detection system (Pierce). Membranes were stripped and re-probed with anti-GAPDH (Sigma) and anti-Lamin A (Santa Cruz Biotechnology) antibodies as control for cytosolic and nuclear fractions, respectively.

Measurement of secreted IL-1 $\beta$. 
IL-1 $\beta$ secretion was quantified in culture supernatants using a commercial ELISA (BD Biosciences) at least in triplicates. Briefly, primary macrophages or THP-1 cells were treated with $E$. coli total RNA $(2 \mu \mathrm{g} / \mathrm{ml}), 2 \times 10^{5} \mathrm{cfu} / \mathrm{ml}$ of heat killed Legionella pneumophila (HKLP) or $2 \times 10^{5} \mathrm{cfu} / \mathrm{ml}$ of heat killed Staphylococcus aureus (HKSA) (InvivoGen) in 12-well dishes for 16 hours and the cleared culture supernatant was analyzed by ELISA. HEK293 cells were grown in collagen Itreated 12-well culture dishes and co-transfected with plasmids encoding mouse pro-IL-1 $\beta$, pro-caspase-1, NLRP3 ${ }^{\mathrm{R} 260 \mathrm{~W}}$, ASC, or NLS-ASC. 24 hours posttransfection, culture media were replaced, collected 36 hours post transfection, clarified, and IL-1 $\beta$ activated by reconstituted inflammasomes was analyzed by mouse IL-1 $\beta$ ELISA according to the manufacturer's protocol [Dorfleutner 2007a,Dorfleutner 2007b,Stehlik 2003].

\section{Statistical methods.}

A standard two-tailed $t$-test was used for statistical analysis; $P$ values of 0.05 or less were considered significant.

\section{RESULTS}

\section{Nuclear localization of ASC in resting monocytes}

ASC has previously been shown to localize diffusely throughout the cell (Fig. $1 A$, upper panel), as well as to form characteristic aggregates (Fig. 1A, middle panel) 
[Masumoto 1999]. In addition, THP-1 cells stably expressing GFP-ASC, predominantly exhibited nuclear distribution of GFP-ASC, as determined by nuclear counter staining (Fig. 1A, lower panel). However, most studies to date have relied upon over-expression of ASC. To clarify the localization of ASC and its implications on the activity of inflammasomes, we studied the subcellular localization of endogenous ASC in monocytes and macrophages, where it is essential for inflammasome function [Mariathasan 2004,Yamamoto 2004]. First we examined ASC subcellular localization by immunofluorescence microscopy in primary human peripheral blood monocytes (Fig. $1 B, 1^{\text {st }}$ panel) and a panel of human monocytic cell lines, including HL-60 (Fig. 1B, $2^{\text {nd }}$ panel), U-937 (Fig. 1B, $3^{\text {rd }}$ panel) and THP-1 cells (Fig. $1 B, 4^{\text {th }}$ panel). Without exception, all cells showed nuclear localization of ASC, emphasizing that endogenous ASC localized to the nucleus in monocytes. Immunostaining was performed with different anti-ASC antibodies, including commercially available mouse monoclonal (U-937) or rabbit polyclonal (HL-60) antibodies or a custom raised rabbit polyclonal anti-ASC antibody (THP-1 and primary monocytes). To further demonstrate specificity of nuclear ASC staining, we performed an ASC peptide competition assay. The custom raised polyclonal anti-ASC antibody was incubated with a 1000 -fold molar excess of the peptide used initially for immunization, before being employed in immunostaining of THP-1 cells (Fig. 1B, $5^{\text {th }}$ panel). Peptide competition completely abrogated nuclear ASC staining in THP-1 cells. These ASC antibodies are highly specificity for ASC without cross- 
reactivity by western blot of THP-1 cell lysates (Fig. 1C) and HEK293 cells that were transfected with myc-tagged ASC or control plasmid (Fig. 1D).

\section{Stimulation-dependent translocation of ASC from the nucleus to the cytosol in monocytes}

Inflammasomes form in response to pathogen and PAMP recognition, and we therefore examined the localization of ASC in primary monocytes and THP-1 monocytes after treatment with E. coli total RNA for 30 minutes, which specifically activates an NLRP3-dependent inflammasome response [Kanneganti 2006a,Kanneganti 2006b]. Contrary to the nuclear localization of ASC observed in resting monocytes (Fig. 1B), immunofluorescence staining of $E$. coli total RNA activated cells revealed a cytosolic distribution of ASC in primary as well as THP1 monocytes (Fig. $2 A$ ). To confirm this observation, proteins from resting and $E$. coli total RNA-treated THP-1 cells were fractionated into nuclear and cytosolic fractions and immunoblotted with ASC-specific antibodies. To control fractionation efficiency, membranes were stripped and re-probed with antibodies specific for the cytosolic GAPDH and the nuclear Lamin A proteins, respectively

(Fig. 2B). Although the control proteins clearly indicated that the fractionation procedure was highly efficient, ASC was also seen in the cytosol in resting cells, which was not visible by immunofluorescence microscopy and was likely caused by nuclear leakage of the small ASC protein during aqueous fractionation, which 
is a well-acknowledged problem for small proteins [Gordon 1981,Paine 1983]. ASC is only $22 \mathrm{kDa}$ in size, and therefore retaining more than $50 \%$ of ASC inside the nucleus during fractionation further confirms its predominantly nuclear localization in resting monocytes, and that ASC redistributes from the nucleus to the cytosol following exposure to E. coli total RNA. Therefore, we conclude that PAMPs are capable of inducing translocation of ASC from the nucleus to the cytosol in monocytes.

\section{Formation of cytosolic ASC aggregates in response to inflammatory activation of macrophages}

Monocytes infiltrate sites of infection and differentiate into inflammatory macrophages to aid pathogen clearance and homeostasis. Therefore, we next examined distribution of ASC in primary macrophages, which were either resting or activated with E. coli total RNA for 6 hours. Similar to monocytes, resting macrophages displayed primarily nuclear ASC (Fig. $3 A$, upper panel), while the majority of E. coli total RNA-activated macrophages showed the characteristic ASC-containing perinuclear aggregates (Fig. $3 A$, lower panel), reminiscent of the structures that were observed upon ASC overexpression (Fig. 1A, middle panel). To prove that THP- 1 cells can be used as a model for inflammasome studies, ASC-containing aggregates were also assessed in PMA-differentiated THP-1 macrophages by immunostaining before and after activation with $E$. coli total 
RNA for 6 hours. As observed for primary macrophages, differentiated resting THP-1 macrophages showed nuclear ASC (Fig. 3B, upper panel) and activation with E. coli total RNA for 6 hours caused ASC to form cytosolic aggregates (Fig. $3 B$, middle panel). To demonstrate specificity for the staining of ASC-containing aggregates, we also performed a peptide competition assay with the custom rabbit polyclonal anti-ASC antibody, as shown in figure $1 B$. Peptide competition completely abrogated any ASC aggregate staining (Fig. 3B, lower panel), which confirmed the specificity of the ASC antibody for ASC aggregate staining. Since ASC is the only known adaptor protein for inflammasome mediated caspase-1 activation, ASC-containing aggregate formation may be crucial for caspase-1 activation and consequently, macrophages from $\mathrm{ASC}^{-/-}$mice fail to activate caspase-1 in response to Gram-positive and Gram-negative bacteria [Mariathasan 2004]. Gram-positive but not Gram-negative pathogens are able to induce inflammasomes in an ASC and NLRP3-dependent manner [Kanneganti 2007,Kanneganti 2006b,Mariathasan 2004,Mariathasan 2006]. To demonstrate that the observed response is not only due to a PAMP, THP-1 derived macrophages were infected with heat-killed Gram-positive Staphylococcus aureus (HKSA) for 4 hours, followed by immunofluorescence staining of ASC. Concomitant with NLRP3-dependent pathogen recognition and subsequent inflammasome formation and activation, Gram-positive pathogens also caused ASC aggregate formation (Fig. 3C, upper panel). Additionally, Gram-negative Legionella pneumophilia (HKLP) for which no specific NLR has been defined yet, 
also caused ASC-containing aggregates (Fig. 3C, lower panel). This data suggest that inflammasomes potentially activated by other NLRs are able to trigger ASC aggregate formation, which is therefore not limited to NLRP3.

\section{ASC aggregation is an early response of macrophage activation}

Maturation and release of IL-1 $\beta$ by macrophages occurs rapidly in response to infections. We therefore wanted to investigate a time course of ASC nuclear to cytosolic redistribution and formation of aggregates in response to stimulation with E. coli RNA. THP-1 cells were kept untreated or treated with E. coli total RNA for $0.5,1,6$ and 24 hours, and distribution of ASC was analyzed in these cells by immunofluorescence (Fig. 4). Within 30 minutes of $E$. coli total RNA treatment the majority of cells displayed ASC redistribution from the nucleus to the cytosol, with some cells showing diffuse nuclear and cytosolic distribution. After 1 hour, ASC was cytosolic, with aggregate formation visible in some cells. Longer exposure to E. coli total RNA induced ASC-containing aggregates in the majority of cells and further increased the size of these aggregates, which are still present after $24+$ hours of activation, without any signs of toxicity. Significantly, duration of ASC-containing aggregates required the persistent presence of E. coli total RNA (or LPS or heat killed pathogens, data not shown), since wash-out of $E$. coli RNA after 6 hours of exposure caused a dissociation of aggregates with some nuclear accumulation of ASC visible after 12 hours (Fig. 4, 
bottom panel). This observation suggested that ASC redistribution and aggregation is reversible, which could indicate a function in host response to infections and other stress situations.

\section{Caspase-1 and NLRP3 co-localize with ASC to aggregates}

Inflammasomes are multi protein complexes consisting of at least an NLR protein, ASC, and caspase-1 [Martinon 2002]. Over-expression studies suggested that many ASC-interacting proteins co-localize to aggregates of overexpressed ASC. We find that, in response to pathogen infection, macrophages display a 4 to $6 \mu \mathrm{m}$ ring shaped perinuclear structure, which contains endogenous ASC (Fig. 5A). Upon compiling of 20 images along the z-axis, a 3 dimensional image revealed that this structure is spherical with a hollow center (Fig. 5B). To characterize localization of additional inflammasome proteins, immunostaining for caspase-1 and NLRP3 was performed. In resting THP-1 macrophages caspase-1 localized to the nucleus and to small vesicular structures in the cytosol (Fig. 5C, upper panel), while E. coli total RNA-activation caused partial localization of caspase-1 to perinuclear aggregates (Fig. 5C, lower panel). Co-staining of caspase-1 with ASC showed that both proteins displayed a similar pattern inside the nucleus in resting THP-1 macrophages (Fig. 5D, upper panel), and that both proteins co-localize to aggregates in $E$. coli total RNA-treated cells (Fig. $5 D$, lower panel). NLRP3 $3^{\text {R260W }}$ is a constitutively active 
CAPS-linked mutant, causing ligand-independent recruitment of ASC and caspase-1 [Dowds 2004,Yu 2006]. Co-expression of ASC, pro-caspase-1 and NLRP3 ${ }^{\text {R260W }}$ in HEK293 cells, caused formation of ASC-containing aggregates and co-localization of all three inflammasome proteins in these aggregates (Fig. $5 E)$. We did not obtain specific staining for endogenous NLRP3 in resting cells, which is in agreement with reports that NLRP3 is inducibly expressed in response to pro-inflammatory agents [O'Connor 2003]. Nevertheless, in E. coli total RNA-treated cells, NLRP3 was present in ASC-containing aggregates in some cells, as well as in punctate structures throughout the cytosol (Fig. 5F). Overall, these results demonstrate that macrophages recruit NLRs, ASC and caspase-1 into perinuclear aggregates in response to pathogen infection, providing the intriguing possibility that these aggregates may represent inflammasomes.

\section{ASC aggregate-dependent maturation of IL-1 $\beta$}

To test the hypothesis that these aggregates may represent inflammasomes, we determined IL-1 $\beta$ secretion in cell culture supernatants by ELISA under conditions that cause ASC-containing aggregate formation in E. coli total RNA treated macrophages (Fig. 6A). Secreted IL-1 $\beta$ was significantly elevated in macrophages after E. coli total RNA treatment over untreated control. To directly link elevated levels of released IL-1 $\beta$ to inflammasomes, we generated stable 
THP-1 cells with an shRNA-mediated knock-down of ASC. shRNA ASC or control THP-1 cells were treated with E. coli total RNA, HKLP or HKSA that cause ASC aggregate formation and secreted IL-1 $\beta$ levels were determined by ELISA in culture supernatants. While control cells show a significant increase in secreted IL-1 $\beta$, ASC knock-down cells are completely impaired in the release of IL-1 $\beta$ into culture supernatants (Fig. 6B). The efficiency of ASC knock-down was assessed by immunoblot in cleared lysates of control and ASC knock-down cells (Fig. 6B). Furthermore, wild type cells (GFP negative) form ASC-containing aggregates, as determined by immunofluorescence analysis, whereas ASC knock-down THP-1 cells (GFP-positive) do not show ASC-containing aggregates following E. coli total RNA treatment, further demonstrating the link between ASC-containing aggregates and IL-1 $\beta$ release (Fig. $6 C$ ).

\section{Redistribution of ASC to the cytosol is essential for inflammasome activity}

The significance of cytosolic redistribution of ASC is further emphasized by the cytosolic distribution of the inflammasome target, pro-IL-1 $\beta$, in response to $E$. coli total RNA treatment of THP-1 cells. Consistent with previous reports that show upregulated pro-IL-1 $\beta$ expression in response to infection [Mileno 1995], no IL-1 $\beta$ staining was observed in resting cells (Fig. 7A, upper panel), while cytosolic distribution is present in activated cells (Fig. 7A, lower panel). To further test the hypothesis that ASC cytosolic redistribution is essential for inflammasome 
formation and activity, we altered its intracellular localization. A constitutive nuclear ASC was generated by in-frame fusion of ASC with three tandem repeats of the strong consensus nuclear localization sequence $(\mathrm{NLS})^{3}$ derived from the SV40 large T antigen, which has frequently been employed to target proteins to the nucleus [Lanford 1986,Screaton 2003]. Three copies of the NLS were used to ensure efficient nuclear retention of ASC even after inflammatory stimulation. We chose this approach over mutational analysis because both death domain folds of ASC require correct folding to retain full adaptor function and even point mutations could disrupt the overall structure and function of ASC, which may lead to a wrong interpretation of results, while $\mathrm{NH}_{2}$ terminal fusion of ASC generally does not alter its function (data not shown). To confirm the nuclear localization of this ASC fusion protein (NLS-ASC), HEK293 cells were transfected and localization was compared to wildtype ASC. As shown in Fig. 1A, overexpression of wildtype ASC results in either nuclear/cytosolic localization, or preferentially the formation of perinuclear aggregates (Fig. $1 A$ and $7 B$, upper panel). In contrast, transfection of NLS-ASC showed exclusively nuclear localization, suggesting that the fusion of a strong NLS to ASC was sufficient to retain it inside the nucleus (Fig. $7 B$, lower panel). To determine the functional significance of nuclear versus cytosolic distribution of ASC, we utilized an inflammasome reconstitution assay in HEK293 cells, which are deficient in endogenous inflammasomes, but are able to produces mature IL-1 $\beta$ upon reconstitution of inflammasomes [Dorfleutner 2007a,Dorfleutner 2007b,Stehlik 
2003]. We reconstituted inflammasomes by transient transfection of procaspase-1, pro-IL-1 $\beta$, NLRP3 ${ }^{\text {R260W }}$, and either ASC or NLS-ASC, and determined the amount of secreted IL-1 $\beta$ in culture supernatants. While inflammasomes containing wild type ASC caused processing and release of IL-1 $\beta$, inflammasomes restored with NLS-ASC were not capable of secreting IL-1 $\beta$ into culture supernatants above baseline level (Fig. 7C). Total cell lysates were used to verify expression of all transfected inflammasome proteins by immunoblotting (Fig. 7C). To directly assess inflammasome activity in macrophages, we infected THP-1 cells with recombinant retroviruses encoding ASC or NLS-ASC, which is not targeted by the siRNA, because we introduced three silent point mutations in the siRNA recognition sequence. We used an RFP fusion to conveniently distinguish between endogenous and ectopic ASC by the increased molecular weight, and chose stable pooled cell populations with expression levels comparable to endogenous ASC, as analyzed by immunoblotting (Fig. 7D, right upper panel). Endogenous ASC was knocked down by two pooled siRNAs, and was confirmed by immunoblotting (Fig. 7D, right lower panel). Next, IL-1 $\beta$ release was measured in response to $E$. coli total RNA treatment. While activation of control cells strongly promoted release of IL-1 $\beta$, knockdown of endogenous ASC from wild type cells efficiently reduced IL-1 $\beta$ release, which was restored in RFP-ASC expressing cells. In contrast, expression of NLS-ASC in ASC knockdown cells failed to restore secretion of IL-1 $\beta$ above the level observed in stimulated ASC knockdown cells (Fig. 7D, left panel). These data 
confirmed that nuclear export of ASC is essential for inflammasome activity, since nuclear retention of ASC completely impaired inflammasome-mediated IL$1 \beta$ release. 


\section{DISCUSSION}

In this report we studied the intracellular distribution of the central inflammasome adapter ASC and provide evidence that inducible redistribution of ASC from the nucleus to the cytosol is essential for inflammasome function. While inflammasomes are biochemically characterized, no detailed studies have been undertaken to visualize endogenous inflammasomes. Over-expression studies result predominantly in the formation of aggregates, referred to as specks [Fernandes-Alnemri 2007,Masumoto 1999]. However, ASC-containing aggregates were believed to represent artificial results driven by enforced overexpression causing self-aggregation of ASC. We observed nuclear localization of ASC in monocytes and macrophages with several anti-ASC antibodies recognizing different epitopes, thus excluding epitope masking as an explanation for our results.

Nuclear localization of ASC, however, cannot explain its essential function as an adaptor protein for inflammasome-mediated caspase-1 activation, which occurs in the cytosol of cells [Brough 2007]. We find cytosolic accumulation of ASC in response to purified PAMPs as well as heat-killed Gram negative and Gram positive bacteria, suggesting that this response is part of the host defense to clear pathogens. Redistribution of ASC from the nucleus to the cytosol can be observed as early as 30 minutes post-stimulation, while longer stimulation caused the aggregation of ASC into structures that resembled the specks 
observed by enforced over-expression of ASC, including the perinuclear localization and the spherical morphology with a hollow center. Additionally, ASC-containing aggregates appear to grow in size over time. To our knowledge, we provide the first evidence of host defense-mediated assembly of ASCcontaining aggregates in macrophages. We propose that inducible redistribution of ASC from the nucleus to the cytosol is required for its interaction with activated NLRs to form caspase-1-activating inflammasomes. ASC-containing aggregates not only assembled in response to the NLRP3 ligands bacterial RNA, and S.aureus, but also in response to Gram negative bacteria and other PAMPs (data not shown), suggesting that other NLRs also aggregate with ASC, consistent with the requirement of ASC for inflammasome-mediated activation of caspase-1 in response to Gram negative and Gram positive pathogens [Mariathasan 2004].

Caspase- 1 activation can induce pyroptosis, which is a form of programmed cell death associated with antimicrobial responses in inflammation, which results in osmotic lysis of cells [Fink 2006]. A caspase-1 and GFP-ASC containing protein complex termed pyroptosome has recently been proposed to facilitate pyroptosis through self-oligomerization of ASC in response to potassium depletion [Fernandes-Alnemri 2007]. However, other reports did not find a link between ASC and pyroptosis [Suzuki 2007]. NLRP3 and ASC have also been implicated in caspase-1 and IL-1 $\beta$-independent pyronecrosis [Willingham 2007]. Thus, the role of ASC and caspase-1 containing complexes in pyroptosis and pyronecrosis is still unclear. We propose that ASC may participate in different 
multi-protein complexes that facilitate either effective IL-1 $\beta$ secretion (inflammasomes) or cell death (pyroptosomes, pyronecrosomes). ASC has also been shown to localize to mitochondria in response to genotoxic insult, which is essential to facilitate p53-mediated Bax translocation to mitochondria and subsequently apoptosis in fibroblasts, hence participating in yet another signaling complex [Ohtsuka 2004]. This complex could be specific to DNA damage in fibroblasts.

Although we do not understand yet what the determining factor for such different outcomes is in macrophages, we noticed significant differences in the appearance and functional properties of these multi-protein complexes. Endogenous ASC aggregates in response to NLR activation are 4-6 $\mu \mathrm{m}$ compared to the smaller $(\sim 1 \mu \mathrm{m})$ and more compact aggregates (specks) caused by over expression of ASC and potassium depletion [Fernandes-Alnemri 2007], or that form in response to cytotoxic treatment of macrophages (data not shown). In spite of aggregate formation, cells do not show any sign of cell death for at least 48 hours, which is in sharp contrast to the rapid cell death in response to the formation of GFP-ASC-containing pyroptosomes [Fernandes-Alnemri 2007]. ASC-containing aggregates are reversible, which is contrary to structures causing cell death, because removal of the activating ligand by washing in culture medium resulted in dissociation of ASC-containing aggregates and diffuse cytoplasmic and even nuclear re-distribution of ASC. Furthermore, these complexes contain caspase- 1 and NLRs, though caspase- 1 co-localization with 
ASC was more frequently detectable than co-localization with NLRP3.

Ultrastructural studies performed by electron microscopy and molecular approaches investigating the release of IL-1 $\beta$ from activated monocytes also did not find any signs of cell death during IL-1 $\beta$ release, and suggested a secretory mechanism that does not involve apoptosis, cell death, or surface membrane lysis [Rubartelli 1990,Singer 1995].

Since conditions that induced ASC aggregates also caused maturation of IL-1 $\beta$ and IL-18, these ASC-containing aggregates could potentially represent inflammasomes. Inflammasomes have been biochemically characterized as large, inducible protein aggregates, consisting of an NLR, ASC, and procaspase-1 [Martinon 2002]. Other proteins can be associated with some inflammasomes. Thus, ASC-containing aggregates might provide a scaffold for inflammasome proteins to interact and to promote caspase-1 activation. Activation of NLRs causes their unfolding and NTP-mediated oligomerization, resulting in pentameric and heptameric aggregates in vitro [Faustin 2007]. Thus, it is feasible that ASC aggregates might denote higher order oligomeric structures of NLRs, as indicated by their larger size. Alternatively, larger aggregates might assemble in vivo, because incorporation of accessory proteins is lacking by in vitro reconstitution. Recombinant NLRP1 pentamers or heptamers are about $20 \mathrm{~nm}$ in diameter [Faustin 2007], while most endogenous ASC aggregates are 4-6 $\mu \mathrm{m}$. Adherence of cells is not required for ASC- 
containing aggregates, as we observed it also in activated monocytes (data not shown).

Fusion of proteins with nuclear localization sequences has been widely employed to prevent redistribution of proteins with nuclear and cytosolic localization. We employed this approach to sequester ASC inside the nucleus of macrophages to prevent ASC export. Nuclear ASC completely abrogated endogenous as well as reconstituted inflammasome-mediated maturation of IL$1 \beta$, directly demonstrating the requirement for ASC redistribution for inflammasome activity. We also noted co-localization of ASC with pro-caspase-1 in the nucleus of resting macrophages, suggesting that both proteins might preassemble in the nucleus of cells even before oligomerization with activated NLRs in the cytosol. In addition, both also localized to ASC-containing aggregates in activated macrophages. Different localizations have been reported for caspases1 , including cytosolic, vesicular, nuclear, as well as the inner and outer face of plasma membranes, which likely reflect different activation states of cells [Mao 1998,Nakagawara 1997 ,Shikama 2001,Singer 1995]. Moreover, caspase-1 is responsible for its own secretion alongside its cytokine substrates [Keller 2008,Martinon 2002]. Nuclear caspase-1 has been suggested to mediate apoptosis in tumor cells, but we did not observe apoptosis in macrophages, in spite of its nuclear localization [Mao 1998]. This is supported by our observation that resting macrophages showed nuclear caspase-1, while a cytosolic to nuclear 
translocation occurs in neuroblastomas undergoing apoptosis [Nakagawara 1997].

Whether ASC is only physically separated inside the nucleus to prevent its spontaneous recruitment to NLRs and subsequent secretion of inflammatory cytokines, or whether ASC performs inflammasome-independent functions inside the nucleus, warrants further investigations. The related protein FADD, which links death receptors to caspase-8 activation in the cytosol, has been recently shown to localize to the nucleus in non-apoptotic cells, and its export is required for caspases-8 activation [Gomez-Angelats 2003,Screaton 2003]. Nuclear and cytosolic FADD is involved in genome surveillance and death receptor-mediated apoptosis, respectively. Nuclear FADD further regulates mitosis and proliferation of T-cells [Alappat 2005]. Likewise cytosolic TRADD, another death domain foldcontaining adapter protein, participates in death receptor-mediated apoptosis, while nuclear localized TRADD mediates PML and p53-mediated apoptosis and interacts with STAT1- $\alpha$ to modulate IFN- $\gamma$ signaling in macrophages [Morgan 2002,Wesemann 2004].

Recent evidence suggested a crosstalk between Toll like receptors $\left(\mathrm{TLRs}^{3}\right.$ and the NLR system during host defense. Pathogen recognition by TLRs is required for up-regulation of inflammasome components and substrates, while subsequently the NLR system is essential for processing of the cytokine precursors. Our data indicate another layer of complexity in the regulation of IL- 
$1 \beta$ and IL-18 maturation, where pathogen recognition initially triggers nuclear to cytosolic redistribution of the NLR adaptor ASC, which then links NLRs to caspase-1 activation in inflammasomes (Fig. 8). While the molecular mechanism of ASC cytosolic redistribution is currently elusive, it potentially could be targeted for prevention of inflammasome-mediated inflammatory cytokine secretion in patients with periodic fever syndromes and other inflammatory conditions. 


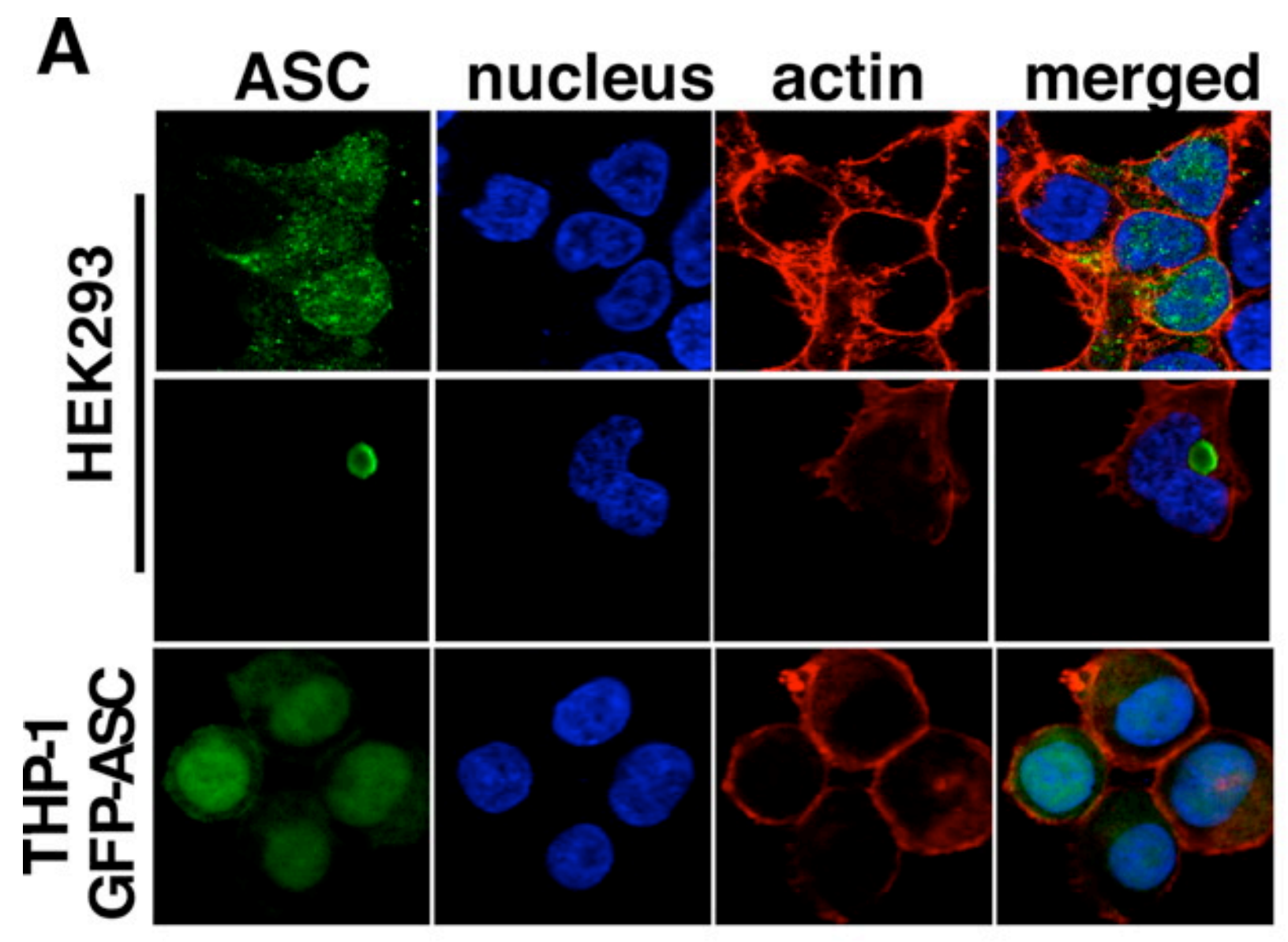

Figure 1. Nuclear localization of ASC.

(A) HEK293 cells were transiently transfected with myc-ASC, fixed, and immunostained with anti-myc and Alexa Fluor 488-conjugated antibodies, while GFP-ASC expressing THP-1 cells were fixed and cytospun onto glass slides. ASC either localized diffusively throughout the cell (upper panel) or into a perinuclear aggregate (middle panel), while stable expression of GFP-ASC in THP-1 cells showed nuclear localization (lower panel). 


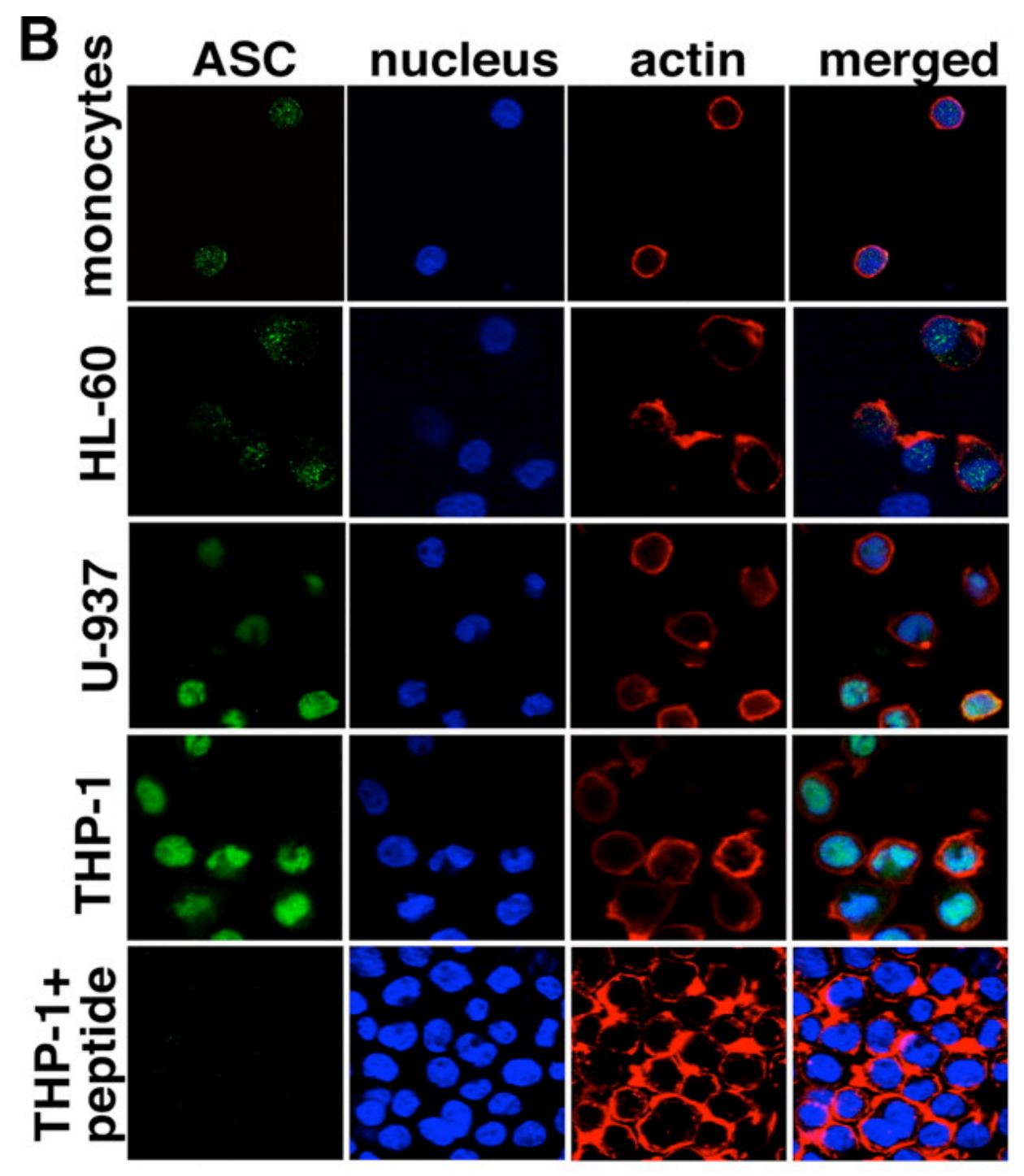

(B) Subcellular localization of endogenous ASC was examined in primary human monocytes and several monocytic cell lines. Cells were fixed and ASC was immunostained with monoclonal antibodies from MBL (monocytes) $\left(1^{\text {st }}\right.$ panel), and polyclonal antibodies from Chemicon, Calbiochem, and custom raised (HL60 , U-937, THP-1) $\left(2^{\text {nd }}, 3^{\text {rd }}\right.$, and $4^{\text {th }}$ panel). THP-1 cells were also immunostained in the presence of a 1000x molar excess of the ASC-specific peptide that is recognized by the CS3 anti-ASC antibody ( $5^{\text {th }}$ panel). Secondary Alexa Fluor 488-conjugated antibodies were used in combination with ToPro-3 and Alexa Fluor 546-conjugated phalloidin to counter stain nuclei (DNA) and the actin cytoskeleton to outline cells, respectively. Images were acquired by laser scanning confocal microscopy, and the panels from left to right are showing ASC (green), nucleus (blue), actin (red), and a merged image. 


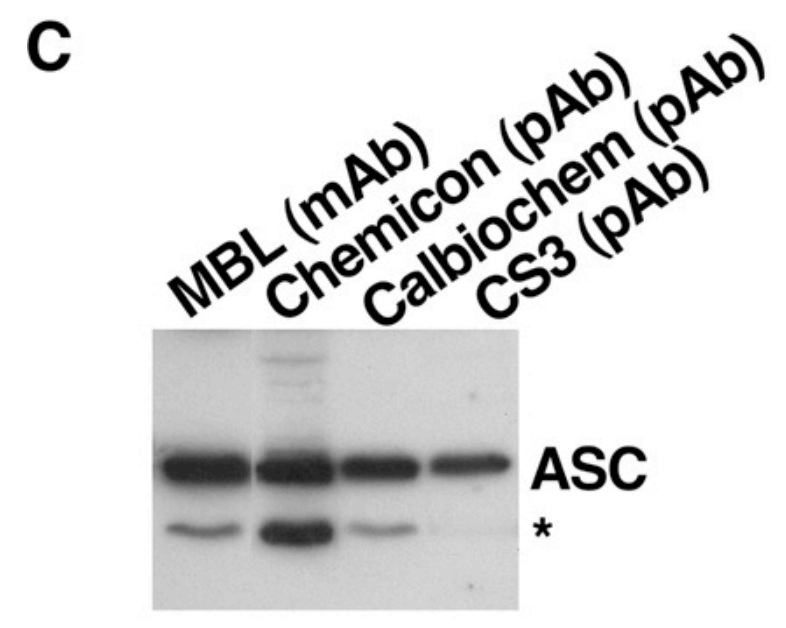

D

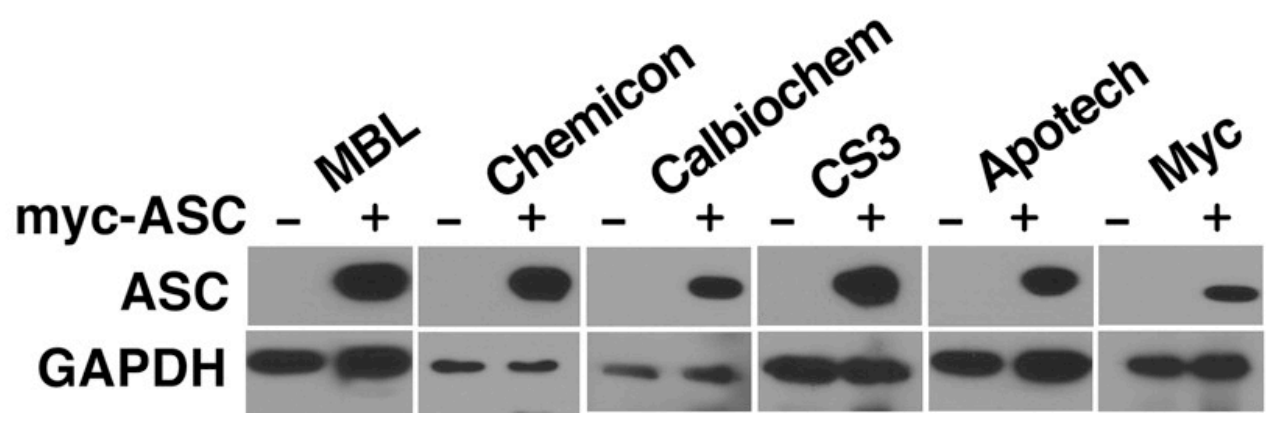

(C) THP-1 lysates $(75 \mu \mathrm{g})$ were analyzed by immunoblot using the anti-ASC antibodies used in immunofluorescence microscopy. An asterisk denotes a splice form of ASC, which is not recognized by our custom-raised polyclonal antiASC antibody (CS3).

(D) HEK293T cells were either mock transfected or transfected with a myctagged ASC expression construct. Cleared cell lysates were analyzed by immunoblot using the anti-ASC antibodies used in immunofluorescence microscopy and anti-myc antibodies (Santa Cruz Biotech) as control. Blots were also stripped and re-probed for GAPDH to demonstrate equal loading. 

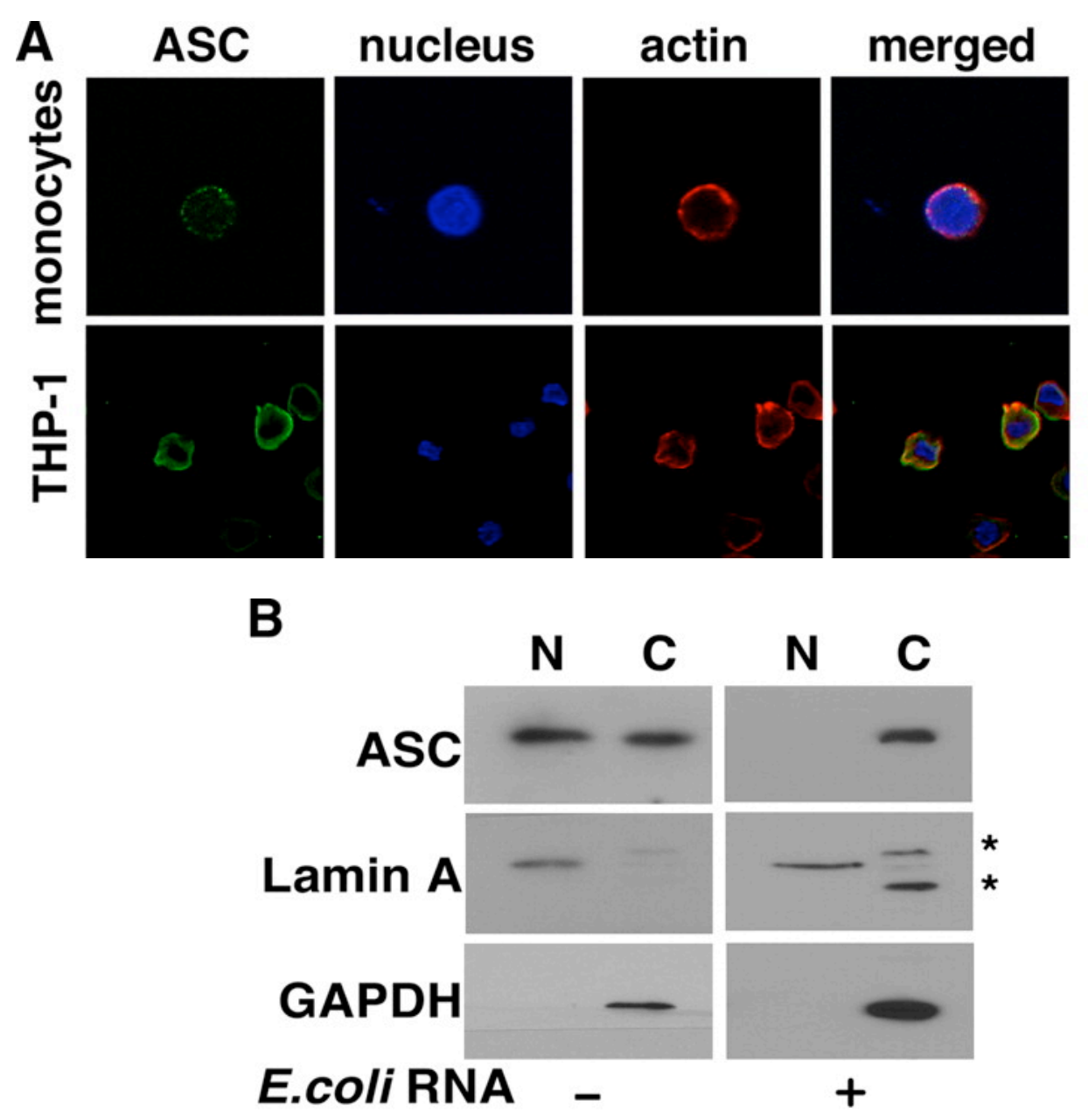

Figure 2. Cytosolic redistribution of ASC in response to inflammatory stimulation of monocytes.

(A) Subcellular localization of ASC was analyzed by immunofluorescence in primary monocytes and THP-1 monocytes following treatment with E. coli total RNA $(2 \mu \mathrm{g} / \mathrm{ml})$ for 30 minutes, using the monoclonal anti-ASC antibody described in Fig. 1. Images were acquired by laser scanning confocal microscopy. Panels from left to right are showing ASC (green), nucleus (blue), actin (red), and a merged image.

(B) Subcellular localization of ASC was determined by subcellular fractionation of control and E. coli total RNA ( $2 \mu \mathrm{g} / \mathrm{ml})$-activated THP-1 cells. $10^{6}$ cells were fractionated by differential centrifugation into nuclear $(\mathrm{N})$ and cytosolic (C) fractions, and protein lysates $(50 \mu \mathrm{g})$ were analyzed by immunoblotting with antiASC and HRP-conjugated secondary antibodies. Fractionation efficiency was controlled by re-probing membranes with anti-GAPDH (cytosolic) and anti-Lamin A (nuclear) antibodies. "denotes two cross reactive proteins in the cytosolic fraction following $E$. coli total RNA treatment. 


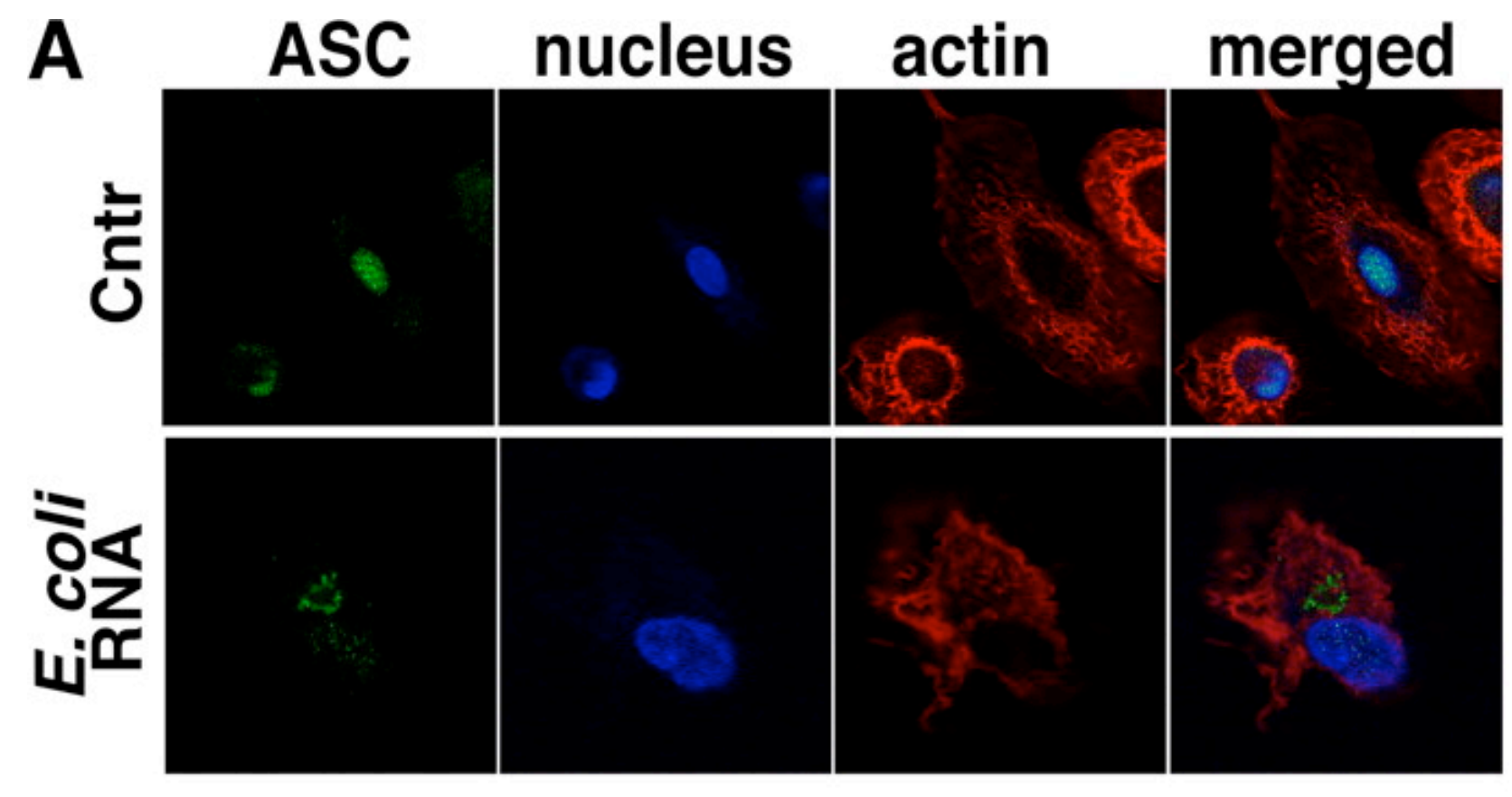

Figure 3. Aggregate formation of endogenous ASC in response to inflammatory stimulation in macrophages. Subcellular localization of ASC was analyzed by immunofluorescence in primary macrophages $(A)$ and PMAdifferentiated THP-1 macrophages $(B, C)$, using mono- and polyclonal anti-ASC and Alexa Fluor 488-conjugated secondary antibodies in combination with ToPro-3 nuclear stain and Alexa Fluor 546 conjugated phalloidin to visualize actin. Images were acquired by laser scanning confocal microscopy. All panels show ASC (green), nucleus (blue), actin (red), and a merged image from left to right.

(A) Primary macrophages, either untreated (upper panel) or E. coli total RNA treated $(2 \mu \mathrm{g} / \mathrm{ml})$ (lower panel), were immunostained using the CS3 polyclonal anti-ASC antibody. 


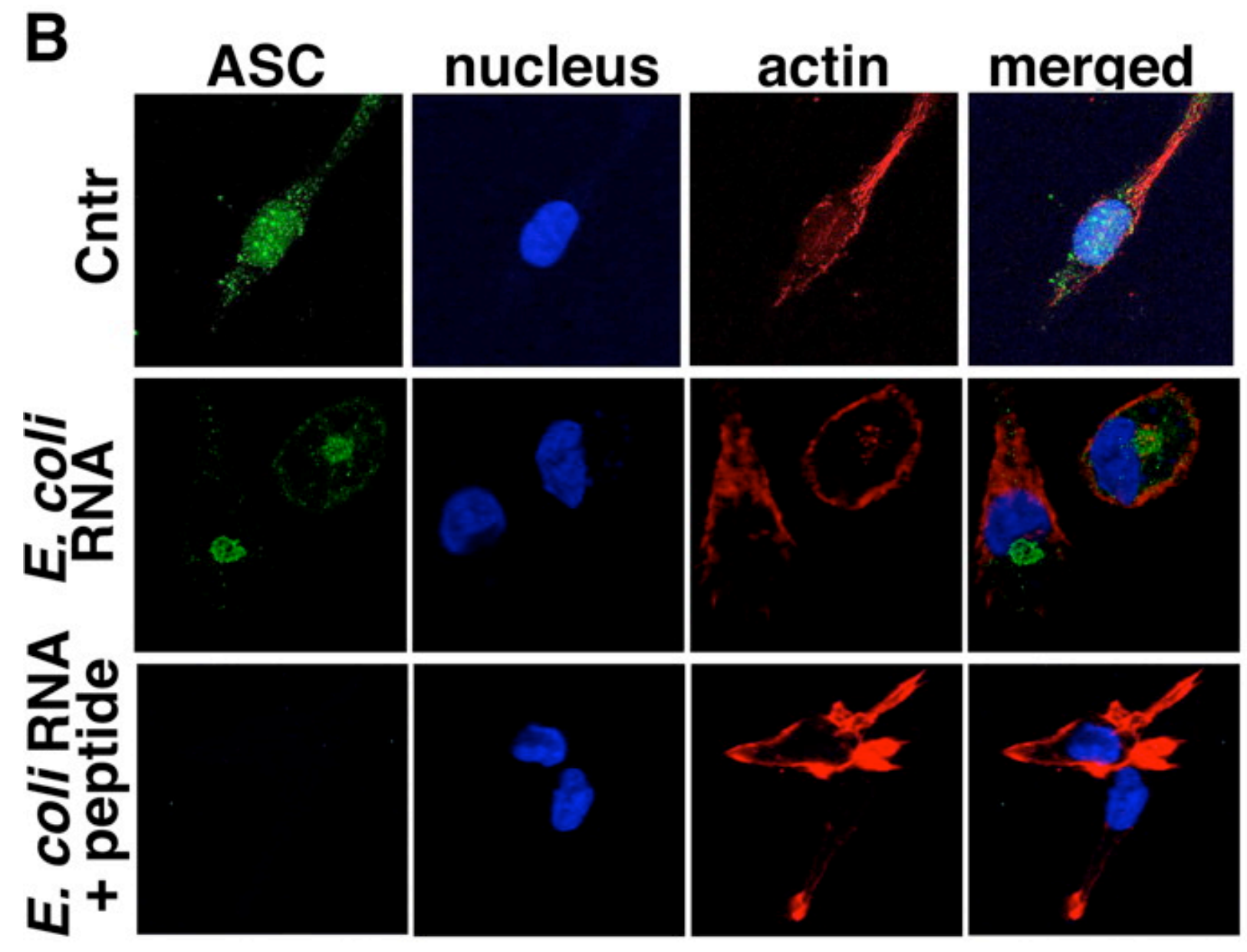

(B) Untreated (upper panel) and E. coli total RNA (2 $\mu \mathrm{g} / \mathrm{ml})$ treated THP-1 macrophages (middle and lower panels) were immunostained using the polyclonal anti-ASC antibody in the absence (middle panel) or in the presence (lower panel) of a 1000x molar excess of the ASC-specific peptide (note the loss of specific ASC aggregate staining in the panel with peptide competition). 


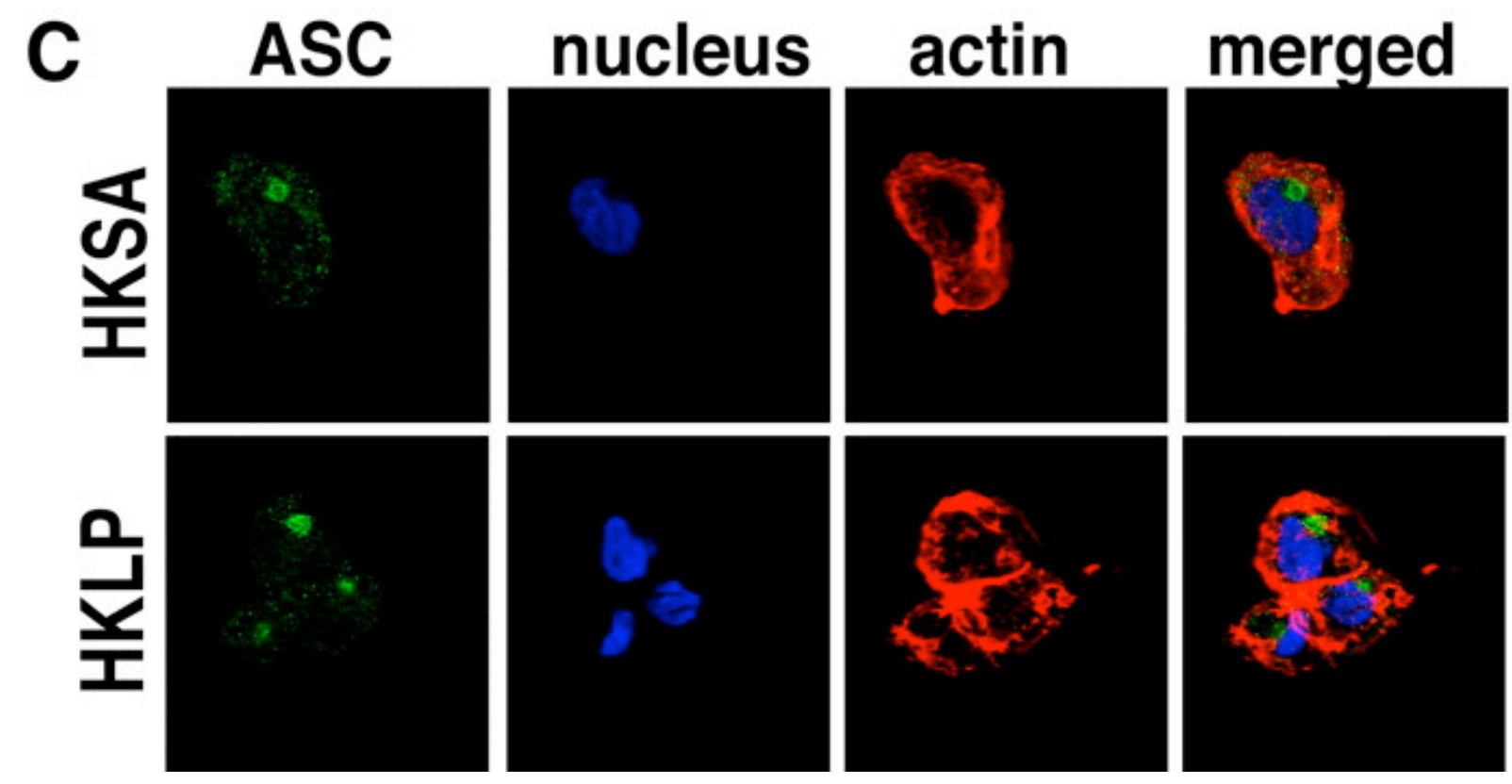

(C) THP-1 macrophages were activated with $2 \times 10^{5} \mathrm{cfu} / \mathrm{ml}$ heat killed Staphylococcus aureus (HKSA) (upper panel) and Legionella pneumophilia (HKLP) (lower panel), and immunostained with the CS3 polyclonal antibody. 


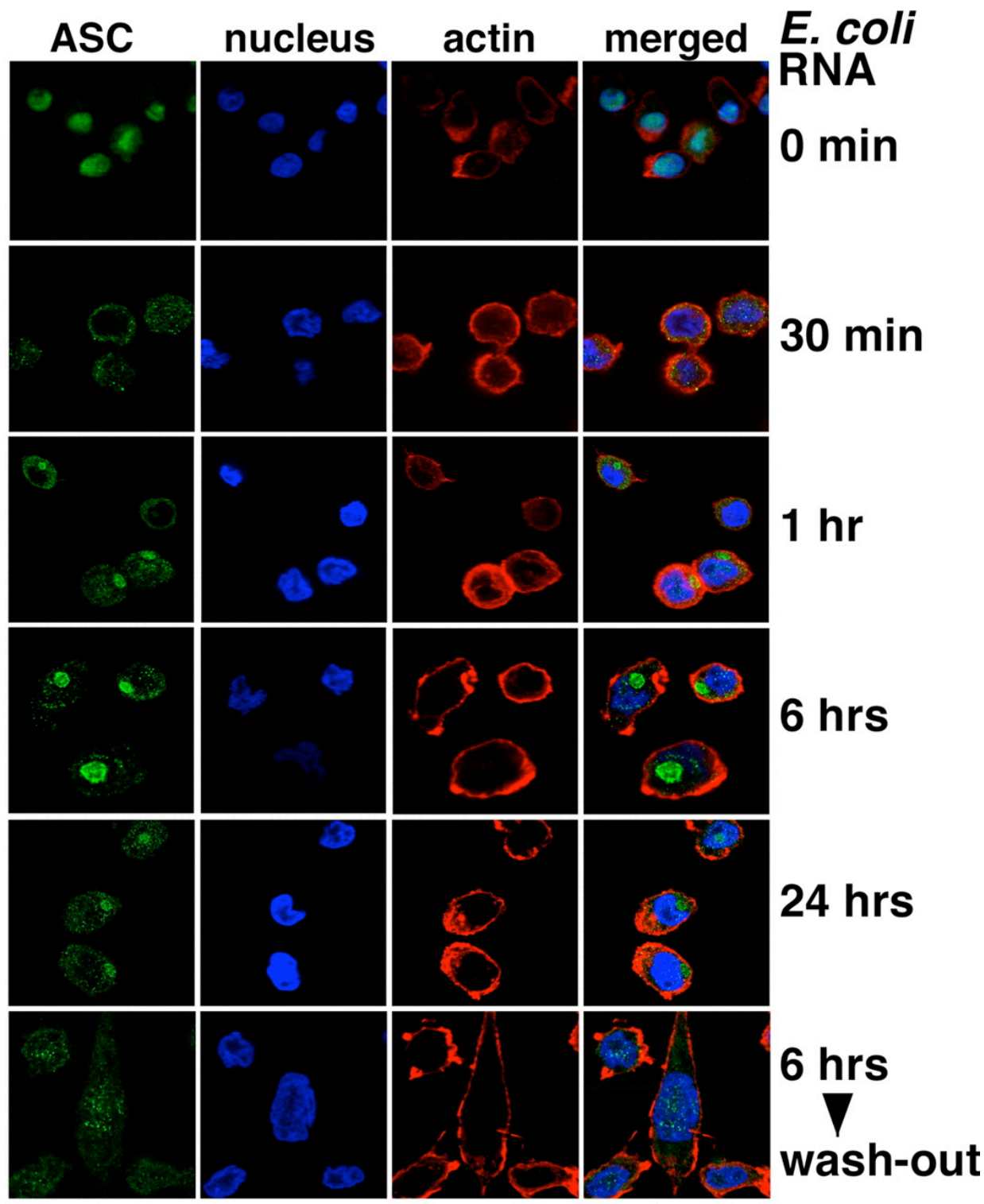

Figure 4. Aggregation of ASC occurs within one hour of inflammatory stimulation of macrophages and depends on the continued presence of the stimulus.

Subcellular localization of ASC in THP-1 macrophages was analyzed by immunofluorescence following stimulation of cells with $E$. coli total RNA $(2 \mu \mathrm{g} / \mathrm{ml})$ for the indicated times. Cells were fixed at 0 minutes, 30 minutes, 1 hour, 6 hours, and 24 hours post-stimulation. In addition, after 6 hours of stimulation, cells were washed extensively and cultured for additional 12 hours in the absence of $E$. coli total RNA. Fixed cells were stained with the polyclonal CS3 anti-ASC, and Alexa Fluor 488-conjugated secondary antibodies. DNA and the actin cytoskeleton were visualized as above. The panels from left to right are showing ASC (green), nucleus (blue), actin (red), and a merged image. 


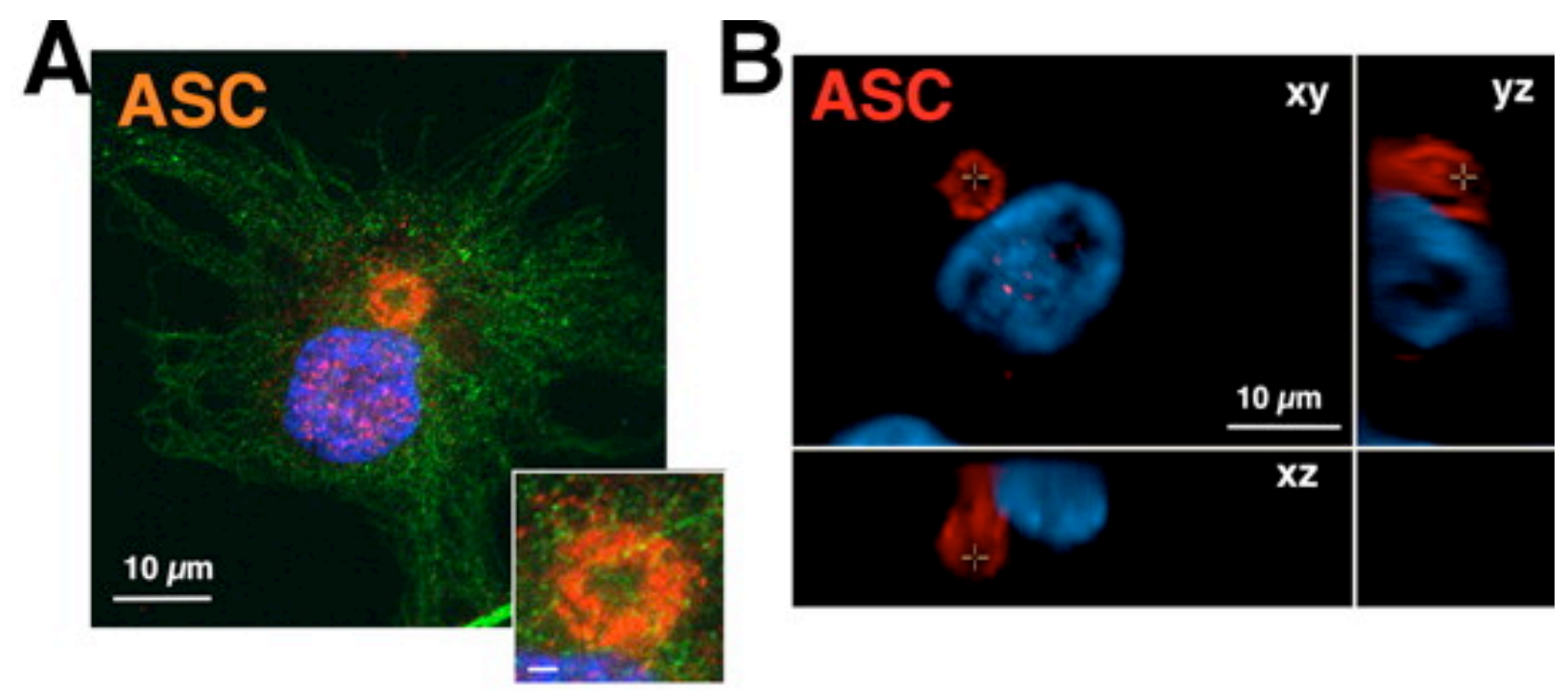

Figure 5. Caspase-1 and NLRP3 co-localize with ASC in aggregates.

All images show PMA-differentiated THP-1 macrophages, except $(E)$, which are HEK293T cells.

(A) Cells were treated with $E$. coli total RNA $(2 \mu \mathrm{g} / \mathrm{ml})$ and immunostained with polyclonal anti-ASC and Alexa Fluor 546-conjugated antibodies (red), monoclonal anti- $\beta$-tubulin and Alexa Fluor 488-conjugated antibodies (green) and the nuclear stain DAPI (blue). The insert in the lower right half highlights the ring-shaped ASC-containing aggregate. The scale bar measures $10 \mu \mathrm{m}$ and 1 $\mu \mathrm{m}$, respectively.

(B) E. coli total RNA (2 $\mu \mathrm{g} / \mathrm{ml})$-treated cells were immunostained with polyclonal anti-ASC and Alexa Fluor 546-conjugated antibodies (red) and DAPI (blue). 20 optical sections at $0.6 \mu \mathrm{m}$ were captured, deconvoluted and assembled into a 3D structure, showing $x y, y z$, and $x z$ views of the aggregate. The scale bar is 10 $\mu \mathrm{m}$. 

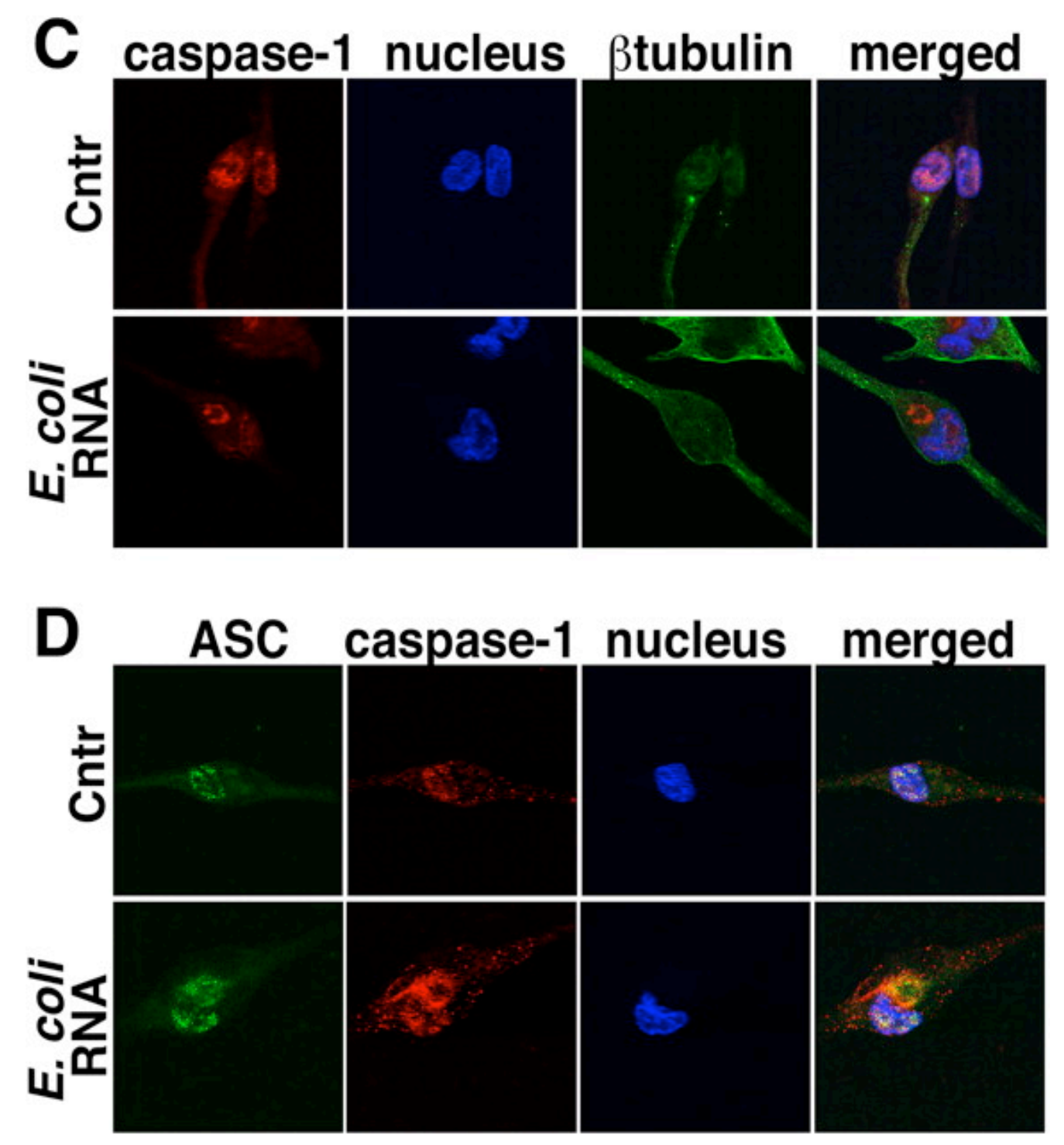

Untreated (upper panel) or E. coli total RNA $(2 \mu \mathrm{g} / \mathrm{ml}$ )-treated cells (lower panel) were fixed and immunostained with $(C)$ polyclonal anti-caspase-1 and monoclonal $\beta$-tubulin and Alexa Fluor 546 and 488-conjugated secondary antibodies, respectively.

(D) Polyclonal anti-ASC and monoclonal anti-caspase-1 antibodies and Alexa Fluor 488- and 546-conjugated secondary antibodies, respectively. DNA was visualized with DAPI, and panels from left to right show $(C)$ caspase-1 (red), nucleus (blue), $\beta$-tubulin (green), and a merged image and (D) ASC (green), caspase-1 (red), nucleus (blue), and a merged image. 


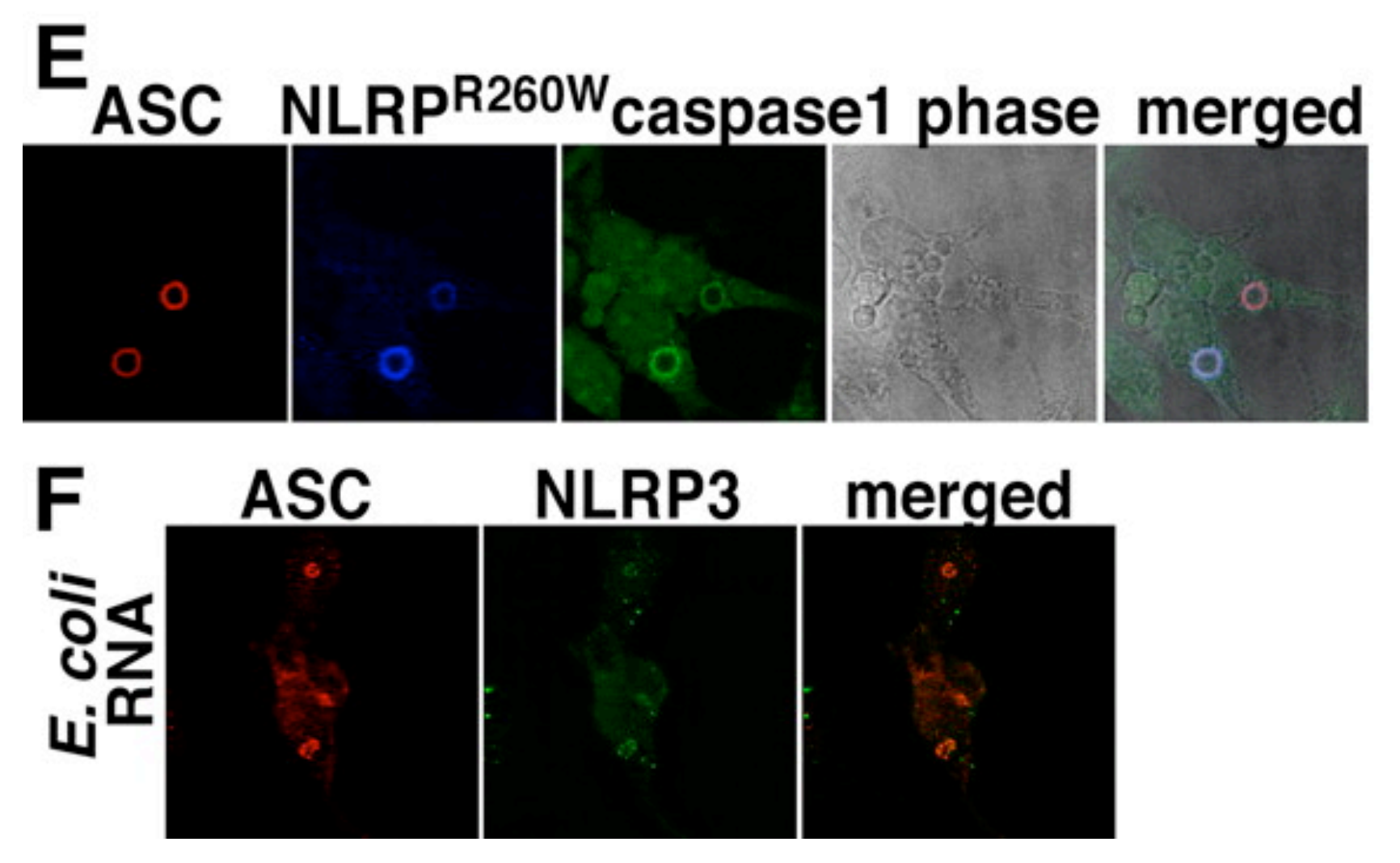

(E) Flag-tagged ASC, HA-tagged caspase-1 and myc-tagged NLRP3 ${ }^{\text {R260W }}$ were transiently transfected into HEK293 cells and immunostained with rabbit antiASC, mouse anti-NLRP3, and rat anti-HA antibodies and Alexa Fluor 546-, 647-, and 488-conjugated secondary antibodies, respectively, to determine colocalization of all three proteins in transfected cells. Panels from left to right show ASC (red), NLRP3 ${ }^{\text {R26OW }}$ (blue), caspases-1 (green), phase, and a merged image.

(F) E. coli total RNA $(2 \mu \mathrm{g} / \mathrm{ml})$-treated THP-1 macrophages were immunostained with rabbit anti-ASC and goat anti-NLRP3 antibodies and Alexa Fluor 488- and 546-conjugated secondary antibodies, respectively. Panels from left to right show ASC (red) and NLRP3 (green) and a merged image. 


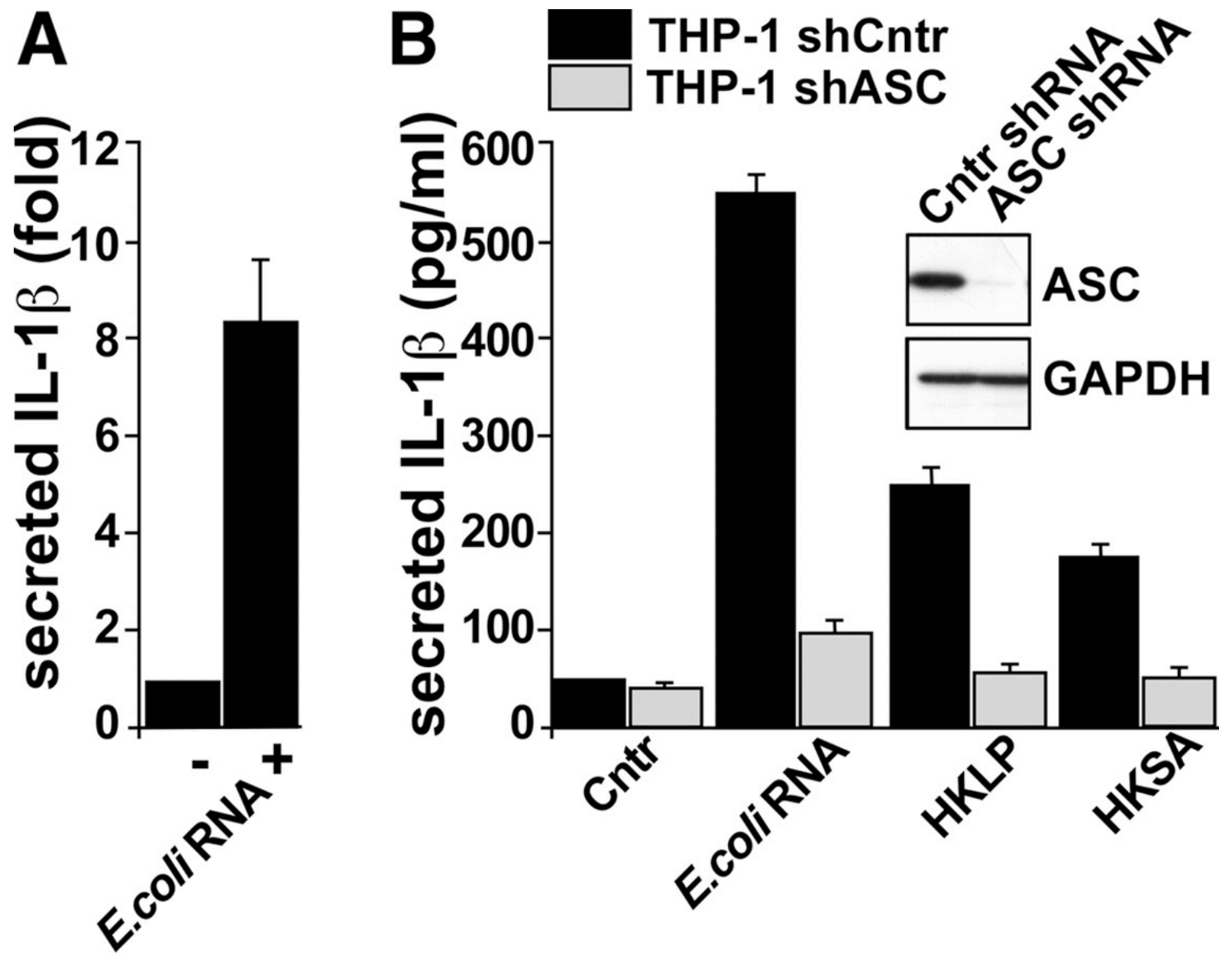

Figure 6. ASC aggregate formation is linked to the maturation of IL-1 3 .

(A) Normalized culture supernatants from resting and E. coli RNA $(2 \mu \mathrm{g} / \mathrm{ml})$ activated primary human macrophages were analyzed by ELISA for released IL$1 \beta$. Results represent an average of two independent experiments, and are presented as fold release compared to untreated macrophages.

(B) Culture supernatants from THP-1 cells stably transfected with either an shRNA targeting ASC (black bars) or a control shRNA targeting luciferase (gray bars) and left either untreated, or treated with $E$. coli RNA $(2 \mu \mathrm{g} / \mathrm{ml})$, HKLP $\left(2 \times 10^{5}\right.$ $\mathrm{cfu} / \mathrm{ml})$ or HKSA $\left(2 \times 10^{5} \mathrm{cfu} / \mathrm{ml}\right)$ were analyzed as above for released IL-1 $\beta$. Cells were previously FACS sorted for GFP expression, which is encoded from the pRNATin-H1.4 (Genscript) shRNA vector backbone. Results represent an average of three independent experiments, and are presented as $\mathrm{pg} / \mathrm{ml}$ of released IL-1 $\beta$. The insert shows an immunoblot of control shRNA and ASC shRNA expressing THP-1 cells for ASC and GAPDH as a loading control. 


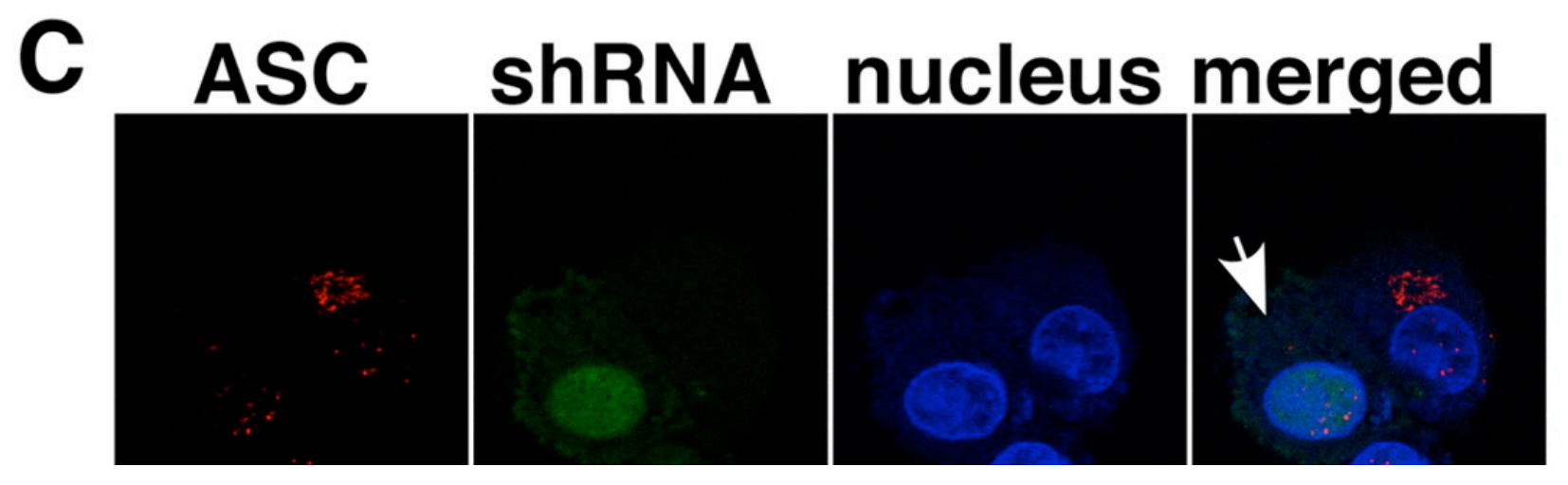

(C) ASC-containing aggregates were analyzed following treatment with E. coli total RNA $(2 \mu \mathrm{g} / \mathrm{ml})$ for 6 hours by immunofluorescence in a mixed population of shRNA ASC transfected cells before FACS sorting for GFP expression of the ASC shRNA. ASC was immunostained with the polyclonal CS3 antibody, and images were acquired by laser scanning confocal microscopy. Panel shows from left to right ASC (red), ASC shRNA (green), nucleus (blue), and a merged image. Note that the cell on the left side (white arrow) encodes the ASC shRNA, as indicated by the GFP positive signal and therefore does not form ASC-containing aggregates, while the cell on the right is GFP negative, thus does not encode the ASC shRNA and therefore shows aggregated ASC. 


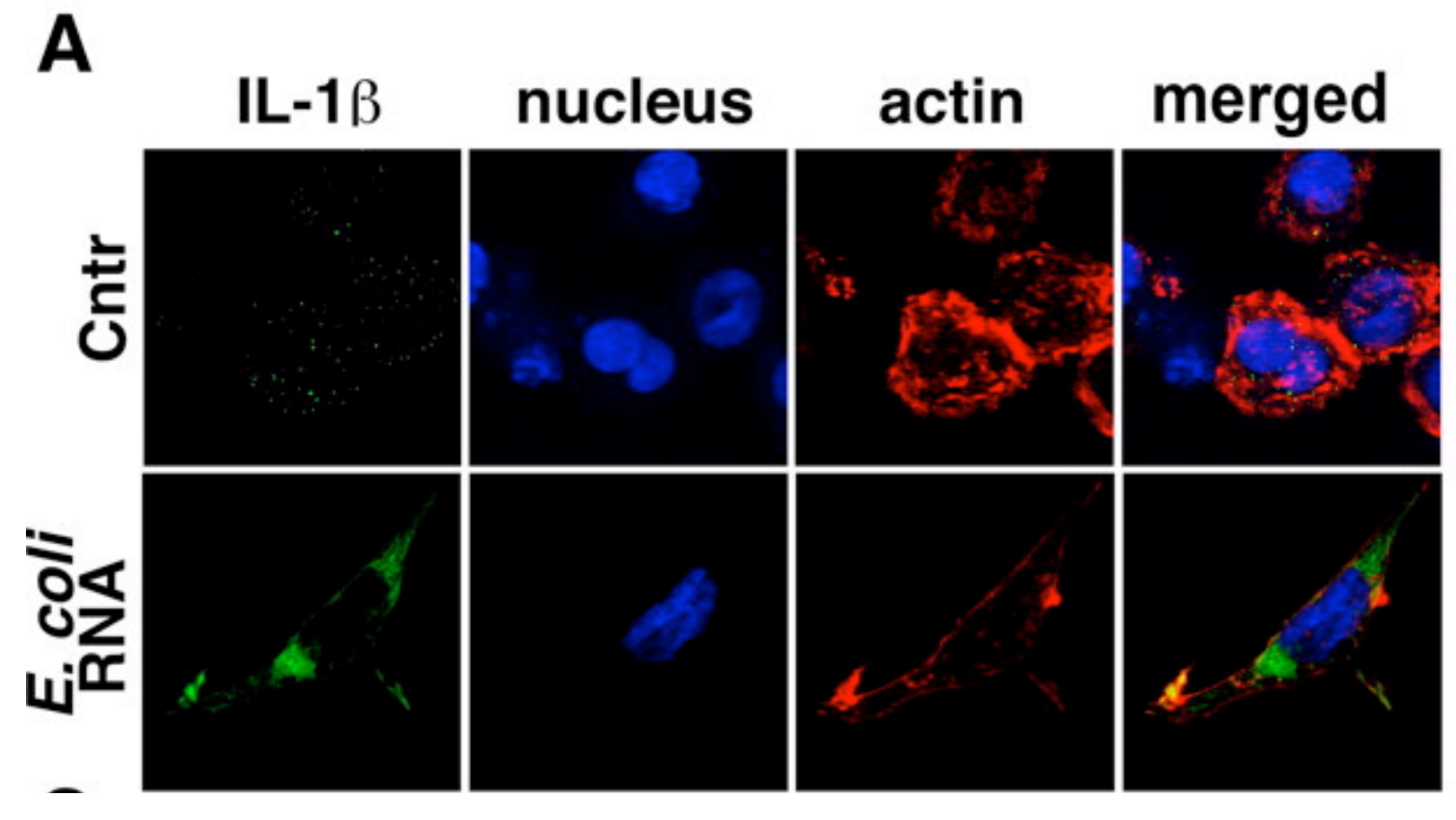

Figure 7. ASC localization to the cytosol is required for inflammasome formation and efficient IL-1 $\beta$ secretion.

(A) Untreated and E. coli RNA-treated THP-1 macrophages were immunostained with anti-IL-1 $\beta$ and Alexa Fluor 488-conjugated secondary antibodies. Nuclei and actin were visualized as above. Panels show from left to right IL-1 $\beta$ (green), nucleus (blue), actin (red), and a merged image. Images were acquired by laser scanning confocal microscopy. 
B

ASC
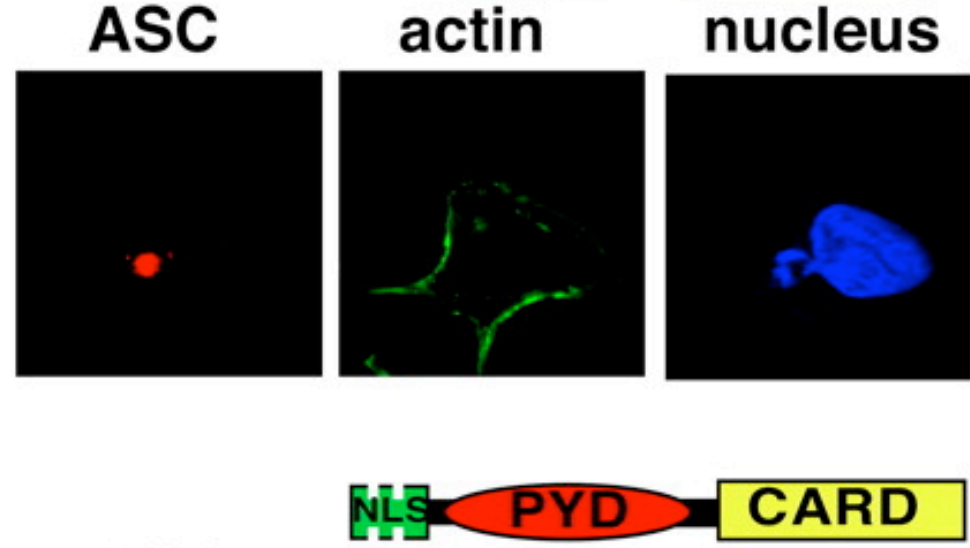

ASC

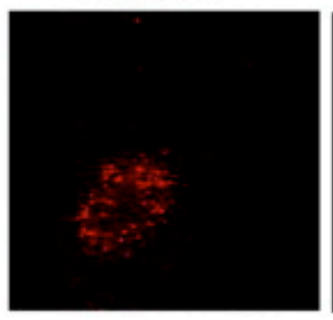

\section{nucleus}

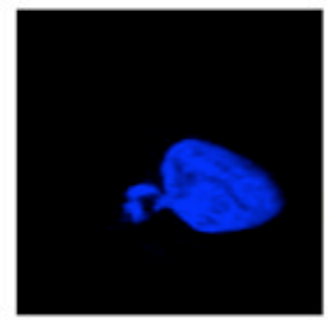

actin

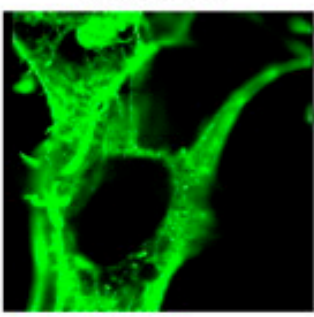

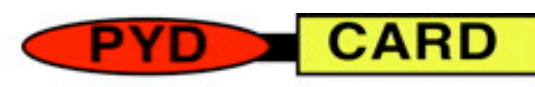

merged

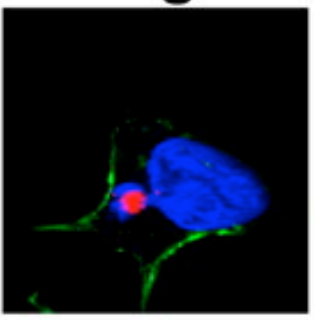

nucleus merged

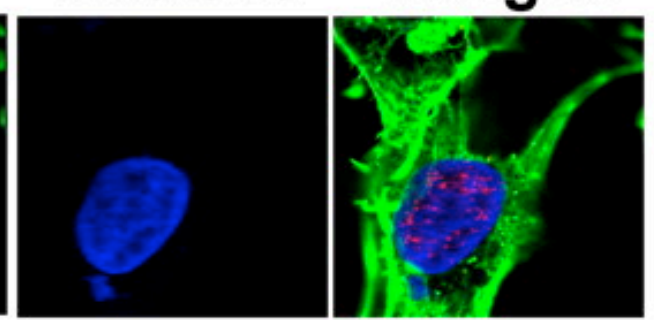

(B) Myc-tagged ASC (upper panel) and myc-tagged NLS-ASC (lower panel) were transiently transfected into HEK293 cells and immunostained with monoclonal anti-myc and Alexa Fluor 546-conjugated secondary antibodies. Nuclei and actin were visualized as above. Panels show from left to right ASC (red), actin (green), nucleus (blue), and a merged image. Images were acquired by laser scanning confocal 


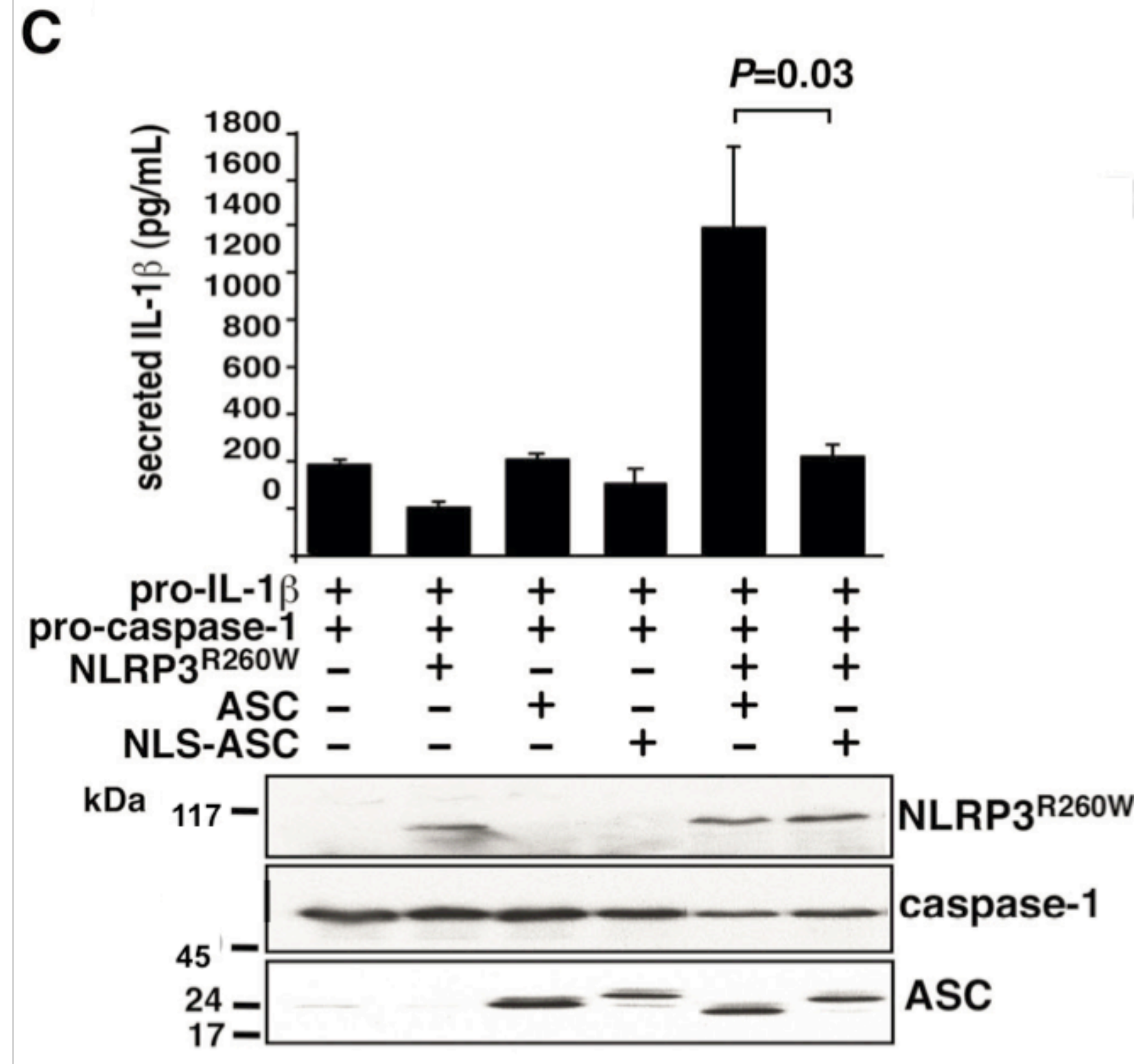

(C) Inflammasomes consisting of a constitutively active NLRP3 ${ }^{\mathrm{R} 260 \mathrm{~W}}$, pro-IL-1 $\beta$, pro-caspase-1, and either ASC or NLS-ASC, were transiently reconstituted in HEK293 cells, as indicated, and inflammasome activity was assayed by analyzing secreted IL-1 $\beta$ by ELISA. Results represent an average of at least three independent experiments $+/-$ SD. Cleared and normalized cellular lysates were analyzed by immunoblot for expression of all transfected inflammasome components, as indicated. 


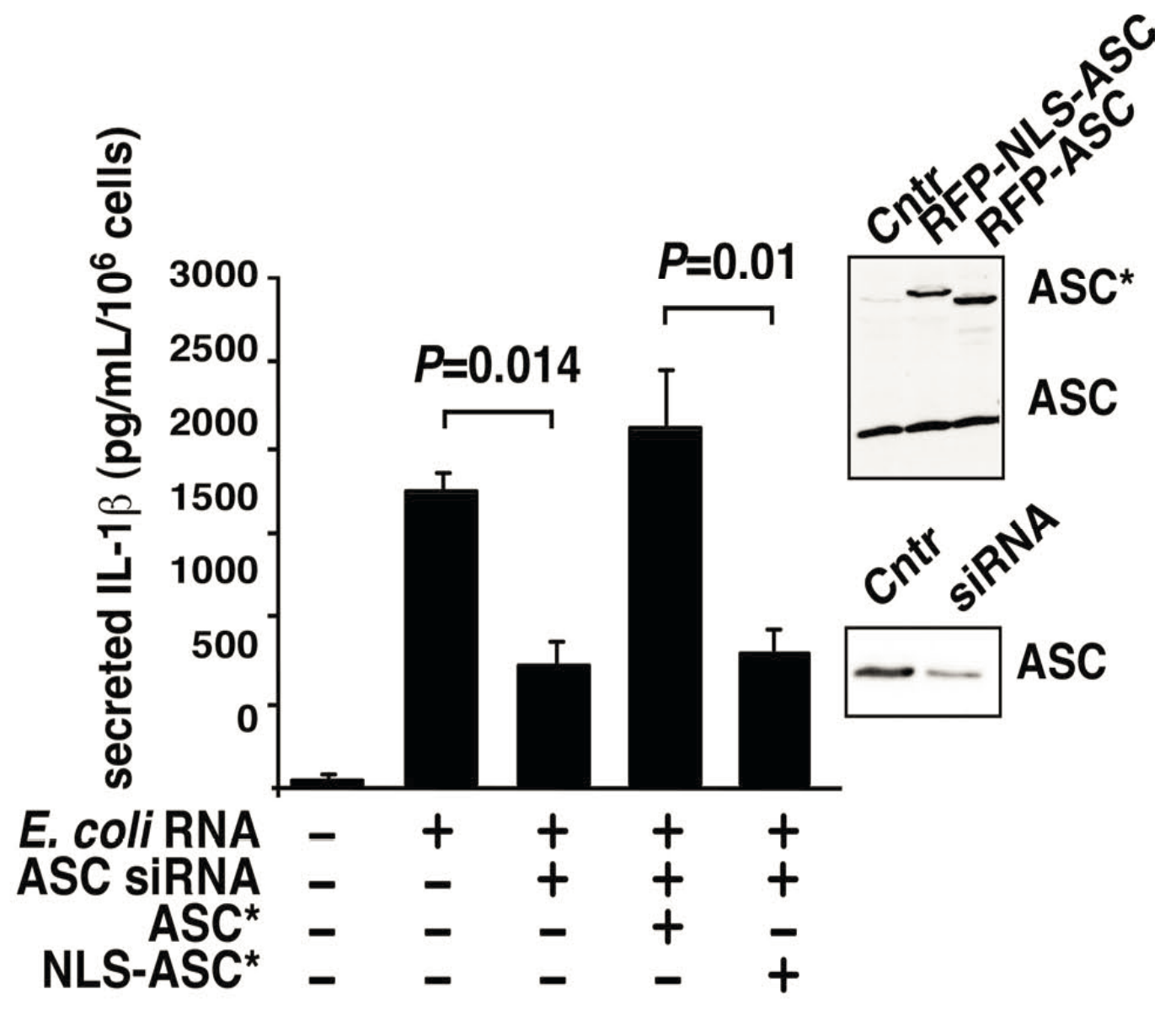

(D) THP-1 cells were stably infected with a VSV-G pseudo-typed recombinant retrovirus encoding either red fluorescent protein (RFP)-fused ASC or RFP-fused NLS-ASC with expression levels comparable to endogenous ASC. Mockinfected, RFP-ASC, and RFP-NLS-ASC expressing cells were transiently transfected with two pooled ASC-specific siRNAs to knock-down expression of endogenous ASC, but not RFP-ASC or RFP-NLS-ASC. Cells were seeded into fresh wells 24 hours post nucleofection, and where indicated treated with LPS and E. coli RNA for 12 hours. Secreted IL-1 $\beta$ was determined in culture supernatants by ELISA and results are presented as $\mathrm{pg} / \mathrm{ml}$ of $10^{6}$ cells, and represent an average of three independent experiments +/- SD. The insert shows stable expression of RFP-ASC and RFP-NLS-ASC compared to endogenous ASC in THP-1 cells (upper panel) and the siRNA-mediated reduction of endogenous ASC (lower panel) by immunoblot. An asterisk denotes expression of ASC and NLS-ASC containing silent point mutations in the sequence recognized by the siRNA preventing its degradation. 


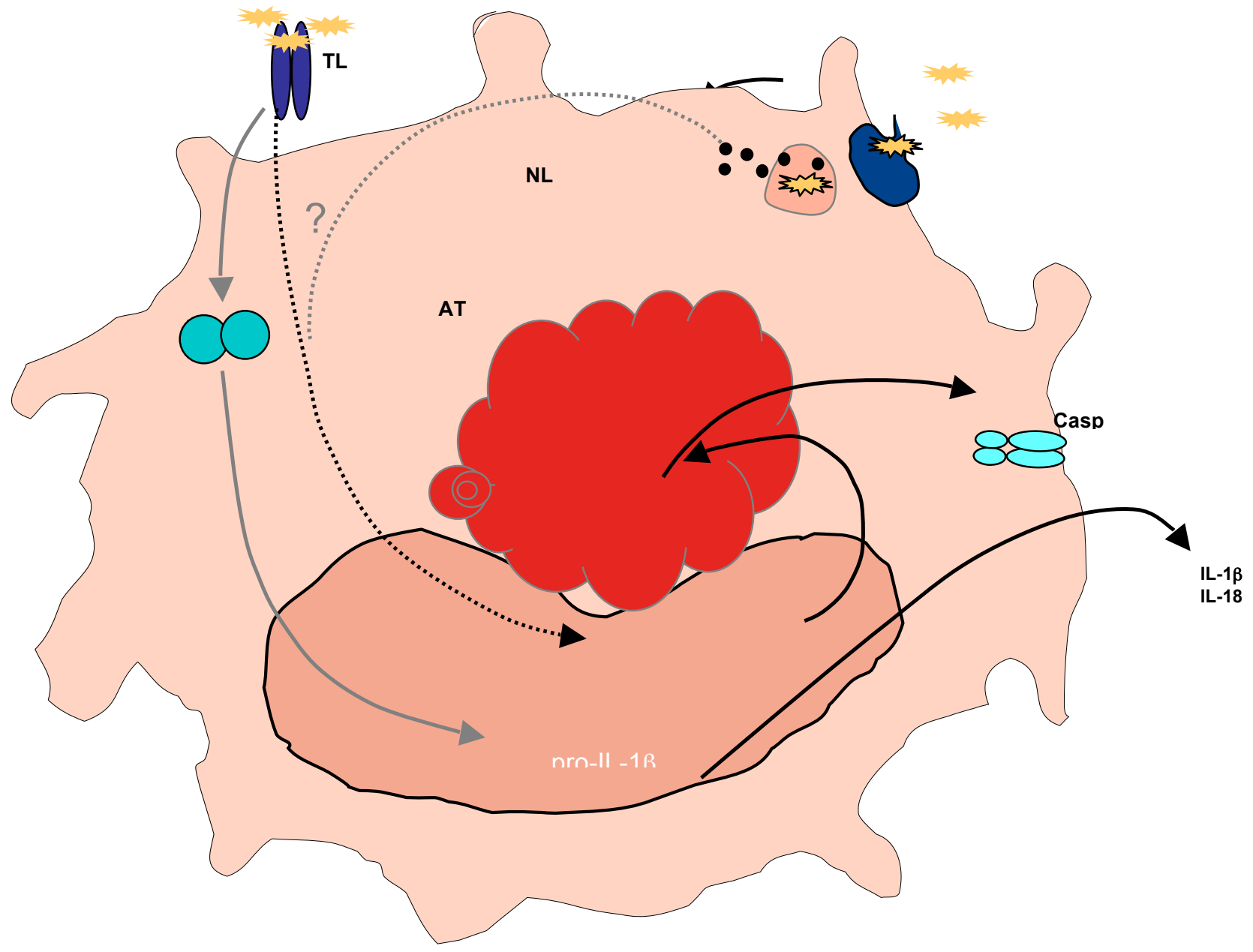

Figure 8. A model for inflammasome formation and activation.

Cross talk between the TLR and NLR system has been proposed, where initial DAMP recognition by TLRs triggers transcriptional up-regulation of pro-IL-1 $\beta$ and other inflammasome components. Subsequently, DAMP recognition by NLRs is required for maturation of pro-IL-1 $\beta$ and pro-IL-18. Our data indicate that DAMP recognition also causes redistribution of ASC from the nucleus to the cytosol by a yet elusive mechanism, which then can be recruited to activated NLRs to assemble inflammasomes. The perinuclear aggregates in activated macrophages contain the core inflammasome proteins and might represent inflammasomes. 


\section{REFERENCES}

Agostini, L., Martinon, F., Burns, K., McDermott, M.F., Hawkins, P.N., and Tschopp, J. (2004) NALP3 forms an IL-1 beta-processing inflammasome with increased activity in Muckle-Wells autoinflammatory disorder. Immunity, 20:319-325. PMC_ID:

Alappat, E.C., Feig, C., Boyerinas, B., Volkland, J., Samuels, M., Murmann, A.E., Thorburn, A., Kidd, V.J., Slaughter, C.A., Osborn, S.L., Winoto, A., Tang, W.J., and Peter, M.E. (2005) Phosphorylation of FADD at serine 194 by CKlalpha regulates its nonapoptotic activities. Mol Cell, 19:321-332. PMC_ID:

Brough, D., and Rothwell, N.J. (2007) Caspase-1-dependent processing of prointerleukin-1beta is cytosolic and precedes cell death. J Cell Sci, 120:772781. PMC_ID:

Church, L.D., Churchman, S.M., Hawkins, P.N., and McDermott, M.F. (2006) Hereditary auto-inflammatory disorders and biologics. Springer Semin Immunopathol, 27:494-508. PMC_ID:

Dinarello, C.A. (1998) Interleukin-1 beta, interleukin-18, and the interleukin-1 beta converting enzyme. Ann. N. Y. Acad. Sci., 856:1-11. PMC_ID:

Dorfleutner, A., Bryan, N.B., Talbott, S.J., Funya, K.N., Rellick, S.L., Reed, J.C., Shi, X., Rojanasakul, Y., Flynn, D.C., and Stehlik, C. (2007a) Cellular PYRIN domain-only protein (cPOP) 2 is a candidate regulator of inflammasome activation. Infect Immun, 75:1484-1492. PMC_ID:

\section{PMC1828547}

Dorfleutner, A., McDonald, S.J., Bryan, N.B., Funya, K.N., Reed, J.C., Shi, X., Flynn, D.C., Rojanasakul, Y., and Stehlik, C. (2007b) A Shope Fibroma virus PYRIN-only protein modulates the host immune response. Virus Genes, 35:685-694. PMC_ID:

Dostert, C., Petrilli, V., Van Bruggen, R., Steele, C., Mossman, B.T., and Tschopp, J. (2008) Innate Immune Activation Through Nalp3 Inflammasome Sensing of Asbestos and Silica. Science. PMC_ID:

Dowds, T.A., Masumoto, J., Zhu, L., Inohara, N., and Nunez, G. (2004) Cryopyrin-induced interleukin 1 beta secretion in monocytic cells: enhanced activity of disease-associated mutants and requirement for ASC. J. Biol. Chem., 279:21924-21928. PMC_ID: 
Duncan, J.A., Bergstralh, D.T., Wang, Y., Willingham, S.B., Ye, Z., Zimmermann, A.G., and Ting, J.P. (2007) Cryopyrin/NALP3 binds ATP/dATP, is an ATPase, and requires ATP binding to mediate inflammatory signaling. Proc Natl Acad Sci U S A, 104:8041-8046. PMC_ID:

Faustin, B., Lartigue, L., Bruey, J.M., Luciano, F., Sergienko, E., Bailly-Maitre, B., Volkmann, N., Hanein, D., Rouiller, I., and Reed, J.C. (2007) Reconstituted NALP1 inflammasome reveals two-step mechanism of caspase-1 activation. Mol Cell, 25:713-724. PMC_ID:

Feldmeyer, L., Keller, M., Niklaus, G., Hohl, D., Werner, S., and Beer, H.D. (2007) The inflammasome mediates UVB-induced activation and secretion of interleukin-1beta by keratinocytes. Curr Biol, 17:1140-1145. PMC_ID:

Fernandes-Alnemri, T., Wu, J., Yu, J.W., Datta, P., Miller, B., Jankowski, W., Rosenberg, S., Zhang, J., and Alnemri, E.S. (2007) The pyroptosome: a supramolecular assembly of ASC dimers mediating inflammatory cell death via caspase-1 activation. Cell Death Differ, 14:1590-1604. PMC_ID:

Fink, S.L., and Cookson, B.T. (2006) Caspase-1-dependent pore formation during pyroptosis leads to osmotic lysis of infected host macrophages. Cell Microbiol, 8:1812-1825. PMC_ID:

Franchi, L., McDonald, C., Kanneganti, T.D., Amer, A., and Nunez, G. (2006) Nucleotide-binding oligomerization domain-like receptors: intracellular pattern recognition molecules for pathogen detection and host defense. $J$ Immunol, 177:3507-3513. PMC_ID:

Gomez-Angelats, M., and Cidlowski, J.A. (2003) Molecular evidence for the nuclear localization of FADD. Cell Death \& Differ, 10:791-797. PMC_ID:

Gordon, J.S., Bruno, J., and Lucas, J.J. (1981) Heterogeneous binding of high mobility group chromosomal proteins to nuclei. J Cell Biol, 88:373-379. PMC_ID:

Jin, Y., Mailloux, C.M., Gowan, K., Riccardi, S.L., LaBerge, G., Bennett, D.C., Fain, P.R., and Spritz, R.A. (2007) NALP1 in vitiligo-associated multiple autoimmune disease. N Engl J Med, 356:1216-1225. PMC_ID:

Kanneganti, T.D., Body-Malapel, M., Amer, A., Park, J.H., Whitfield, J., Taraporewala, Z.F., Miller, D., Patton, J.T., Inohara, N., and Nunez, G. (2006a) Critical role for cryopyrin/Nalp3 in activation of caspase-1 in response to viral infection and double-stranded RNA. J Biol Chem. PMC_ID: 
Kanneganti, T.D., Ozoren, N., Body-Malapel, M., Amer, A., Park, J.H., Franchi, L., Whitfield, J., Barchet, W., Colonna, M., Vandenabeele, P., Bertin, J., Coyle, A., Grant, E.P., Akira, S., and Nunez, G. (2006b) Bacterial RNA and small antiviral compounds activate caspase-1 through cryopyrin/Nalp3. Nature, 440:232-236. PMC_ID:

Kanneganti, T.D., Lamkanfi, M., Kim, Y.G., Chen, G., Park, J.H., Franchi, L., Vandenabeele, P., and Nunez, G. (2007) Pannexin-1-mediated recognition of bacterial molecules activates the cryopyrin inflammasome independent of Toll-like receptor signaling. Immunity, 26:433-443.

PMC_ID:

Keller, M., Ruegg, A., Werner, S., and Beer, H.D. (2008) Active caspase-1 is a regulator of unconventional protein secretion. Cell, 132:818-831. PMC_ID:

Lanford, R.E., Kanda, P., and Kennedy, R.C. (1986) Induction of nuclear transport with a synthetic peptide homologous to the SV40 T antigen transport signal. Cell, 46:575-582. PMC_ID:

Mao, P.-L., Jiang, Y., Wee, B., and Porter, A. (1998) Activation of caspase-1 in the nucleus requires nuclear translocation of pro-caspase-1 mediated by its prodomain. J. Biol. Chem., 273:23621-23624. PMC_ID:

Mariathasan, S., Newton, K., Monack, D.M., Vucic, D., French, D.M., Lee, W.P., Roose-Girma, M., Erickson, S., and Dixit, V.M. (2004) Differential activation of the inflammasome by caspase-1 adaptors ASC and Ipaf. Nature, 430:213-218. PMC_ID:

Mariathasan, S., Weiss, D.S., Newton, K., McBride, J., O'Rourke, K., RooseGirma, M., Lee, W.P., Weinrauch, Y., Monack, D.M., and Dixit, V.M. (2006) Cryopyrin activates the inflammasome in response to toxins and ATP. Nature, 440:228-232. PMC_ID:

Mariathasan, S., and Monack, D.M. (2007) Inflammasome adaptors and sensors: intracellular regulators of infection and inflammation. Nat Rev Immunol, 7:31-40. PMC_ID:

Martinon, F., Burns, K., and Tschopp, J. (2002) The Inflammasome: A molecular platform triggering activation of inflammatory caspases and processing of prolL-1b. Mol. Cell, 10:417-426. PMC_ID:

Martinon, F., Agostini, L., Meylan, E., and Tschopp, J. (2004) Identification of bacterial muramyl dipeptide as activator of the NALP3/cryopyrin inflammasome. Curr Biol, 14:1929-1934. PMC_ID: 
Martinon, F., Petrilli, V., Mayor, A., Tardivel, A., and Tschopp, J. (2006) Goutassociated uric acid crystals activate the NALP3 inflammasome. Nature, 440:237-241. PMC_ID:

Martinon, F., Gaide, O., Petrilli, V., Mayor, A., and Tschopp, J. (2007) NALP Inflammasomes: a central role in innate immunity. Semin Immunopathol, 29:213-229. PMC_ID:

Masumoto, J., Taniguchi, S., Ayukawa, K., Sarvotham, H., Kishino, T., Niikawa, N., Hidaka, E., Katsuyama, T., Higuchi, T., and Sagara, J. (1999) ASC, a novel 22-kDa protein, aggregates during apoptosis of human promyelocytic leukemia HL-60 cells. J. Biol. Chem., 274:33835-33838. PMC_ID:

Mileno, M.D., Margolis, N.H., Clark, B.D., Dinarello, C.A., Burke, J.F., and Gelfand, J.A. (1995) Coagulation of whole blood stimulates interleukin-1 beta gene expression. J Infect Dis, 172:308-311. PMC_ID:

Morgan, M., Thorburn, J., Pandolfi, P.P., and Thorburn, A. (2002) Nuclear and cytoplasmic shuttling of TRADD induces apoptosis via different mechanisms. J Cell Biol, 157:975-984. PMC_ID:

Muruve, D.A., Petrilli, V., Zaiss, A.K., White, L.R., Clark, S.A., Ross, P.J., Parks, R.J., and Tschopp, J. (2008) The inflammasome recognizes cytosolic microbial and host DNA and triggers an innate immune response. Nature, 452:103-107. PMC_ID:

Nakagawara, A., Nakamura, Y., Ikeda, H., Hiwasa, T., Kuida, K., Su, M.S., Zhao, H., Cnaan, A., and Sakiyama, S. (1997) High levels of expression and nuclear localization of interleukin-1 beta converting enzyme (ICE) and CPP32 in favorable human neuroblastomas. Cancer Res, 57:4578-4584. PMC_ID:

O'Connor, W., Jr., Harton, J.A., Zhu, X., Linhoff, M.W., and Ting, J.P. (2003) Cutting edge: CIAS1/cryopyrin/PYPAF1/NALP3/ CATERPILLER 1.1 Is an inducible inflammatory mediator with NF-kappaB suppressive properties. J. Immunol., 171:6329-6333. PMC_ID:

Ogura, Y., Sutterwala, F.S., and Flavell, R.A. (2006) The inflammasome: first line of the immune response to cell stress. Cell, 126:659-662. PMC_ID:

Ohtsuka, T., Ryu, H., Minamishima, Y.A., Macip, S., Sagara, J., Nakayama, K.I., Aaronson, S.A., and Lee, S.W. (2004) ASC is a Bax adaptor and regulates the p53-Bax mitochondrial apoptosis pathway. Nat. Cell. Biol., 6:121-128. PMC_ID: 
Omi, T., Kumada, M., Kamesaki, T., Okuda, H., Munkhtulga, L., Yanagisawa, Y., Utsumi, N., Gotoh, T., Hata, A., Soma, M., Umemura, S., Ogihara, T., Takahashi, N., Tabara, Y., Shimada, K., Mano, H., Kajii, E., Miki, T., and Iwamoto, S. (2006) An intronic variable number of tandem repeat polymorphisms of the cold-induced autoinflammatory syndrome 1 (CIAS1) gene modifies gene expression and is associated with essential hypertension. Eur J Hum Genet, 14:1295-1305. PMC_ID:

Paine, P.L., Austerberry, C.F., Desjarlais, L.J., and Horowitz, S.B. (1983) Protein loss during nuclear isolation. J Cell Biol, 97:1240-1242. PMC_ID:

Rosengren, S., Hoffman, H.M., Bugbee, W., and Boyle, D.L. (2005) Expression and regulation of cryopyrin and related proteins in rheumatoid arthritis synovium. Ann Rheum Dis, 64:708-714. PMC_ID:

Rubartelli, A., Cozzolino, F., Talio, M., and Sitia, R. (1990) A novel secretory pathway for interleukin-1 beta, a protein lacking a signal sequence. EMBO J, 9:1503-1510. PMC_ID:

Screaton, R.A., Kiessling, S., Sansom, O.J., Millar, C.B., Maddison, K., Bird, A., Clarke, A.R., and Frisch, S.M. (2003) Fas-associated death domain protein interacts with methyl-CpG binding domain protein 4: a potential link between genome surveillance and apoptosis. Proc Natl Acad Sci U S A, 100:5211-5216. PMC_ID:

Shikama, Y. (2001) Comprehensive studies on subcellular localizations and cell death-inducing activities of eight GFP-tagged apoptosis-related caspases. Exp Cell Res, 264:315-325. PMC_ID:

Singer, II, Scott, S., Chin, J., Bayne, E.K., Limjuco, G., Weidner, J., Miller, D.K., Chapman, K., and Kostura, M.J. (1995) The interleukin-1 beta-converting enzyme (ICE) is localized on the external cell surface membranes and in the cytoplasmic ground substance of human monocytes by immunoelectron microscopy. J Exp Med, 182:1447-1459. PMC_ID:

Srinivasula, S.M., Poyet, J.-L., Razmara, M., Datta, P., Zhang, Z., and Alnemri, E.S. (2002) The PYRIN-CARD protein ASC is an activating adaptor for Caspase-1. J. Biol. Chem., 277:21119-21122. PMC_ID:

Stehlik, C., Fiorentino, L., Dorfleutner, A., Bruey, J.M., Ariza, E.M., Sagara, J., and Reed, J.C. (2002) The PAAD/PYRIN-family protein ASC is a dual regulator of a conserved step in nuclear factor kappaB activation pathways. J. Exp. Med., 196:1605-1615. PMC_ID: PMC2196065 
Stehlik, C., Lee, S.H., Dorfleutner, A., Stassinopoulos, A., Sagara, J., and Reed, J.C. (2003) Apoptosis-associated speck-like protein containing a caspase recruitment domain is a regulator of procaspase-1 activation. J. Immunol., 171:6154-6163. PMC_ID:

Stehlik, C. (2007a) The PYRIN domain in signal transduction. Curr Protein Pept Sci, 8:293-310. PMC_ID:

Stehlik, C., and Dorfleutner, A. (2007b) COPs and POPs: Modulators of Inflammasome Activity. J Immunol, 179:7993-7998. PMC_ID: 18056338

Stojanov, S., and Kastner, D.L. (2005) Familial autoinflammatory diseases: genetics, pathogenesis and treatment. Curr Opin Rheumatol, 17:586-599. PMC_ID:

Sutterwala, F.S., Ogura, Y., Szczepanik, M., Lara-Tejero, M., Lichtenberger, G.S., Grant, E.P., Bertin, J., Coyle, A.J., Galan, J.E., Askenase, P.W., and Flavell, R.A. (2006) Critical role for NALP3/CIAS1/Cryopyrin in innate and adaptive immunity through its regulation of caspase-1. Immunity, 24:317327. PMC_ID:

Suzuki, T., Franchi, L., Toma, C., Ashida, H., Ogawa, M., Yoshikawa, Y., Mimuro, H., Inohara, N., Sasakawa, C., and Nunez, G. (2007) Differential regulation of caspase-1 activation, pyroptosis, and autophagy via Ipaf and ASC in Shigella-infected macrophages. PLoS Pathog, 3:e111. PMC_ID:

Ting, J.P., Kastner, D.L., and Hoffman, H.M. (2006) CATERPILLERs, pyrin and hereditary immunological disorders. Nat Rev Immunol, 6:183-195. PMC_ID:

Watanabe, H., Gaide, O., Petrilli, V., Martinon, F., Contassot, E., Roques, S., Kummer, J.A., Tschopp, J., and French, L.E. (2007) Activation of the IL1 beta-processing inflammasome is involved in contact hypersensitivity. $J$ Invest Dermatol, 127:1956-1963. PMC_ID:

Wesemann, D.R., Qin, H., Kokorina, N., and Benveniste, E.N. (2004) TRADD interacts with STAT1-alpha and influences interferon-gamma signaling. Nat Immunol, 5:199-207. PMC_ID:

Willingham, S.B., Bergstralh, D.T., O'Connor, W., Morrison, A.C., Taxman, D.J., Duncan, J.A., Barnoy, S., Venkatesan, M.M., Flavell, R.A., Deshmukh, M., Hoffman, H.M., and Ting, J.P. (2007) Microbial pathogen-induced necrotic cell death mediated by the inflammasome components CIAS1/cryopyrin/NLRP3 and ASC. Cell Host Microbe, 2:147-159. PMC_ID: 
Yamamoto, M., Yaginuma, K., Tsutsui, H., Sagara, J., Guan, X., Seki, E., Yasuda, K., Yamamoto, M., Akira, S., Nakanishi, K., Noda, T., and Taniguchi, S. (2004) ASC is essential for LPS-induced activation of procaspase-1 independently of TLR-associated signal adaptor molecules. Genes Cells, 9:1055-1067. PMC_ID:

Yu, J.W., Wu, J., Zhang, Z., Datta, P., Ibrahimi, I., Taniguchi, S., Sagara, J., Fernandes-Alnemri, T., and Alnemri, E.S. (2006) Cryopyrin and pyrin activate caspase-1, but not NF-kappaB, via ASC oligomerization. Cell Death Differ, 13:236-249. PMC_ID: 
Study 2:

Mycoplasma: A novel activator of the inflammasome 


\section{ABSTRACT}

Mycoplasma species are very unique parasitic bacteria, which lack a cell wall. They are the smallest known free-living organisms and are known to cause a large number of organ-specific diseases such as pneumonia and pericarditis. Also, they have been linked to a number of chronic inflammatory diseases. However, in spite of the large number of pathogenic Mycoplasma species, very little is known about how the host immune system detects and responds to infection by these organisms. IL-1beta is a key pro-inflammatory cytokine in the innate immune defense against pathogens. Because of its wide-ranging impacts, the release of IL-1beta is highly regulated, involving a two-step process of transcriptional upregulation of the precursor followed by inflammasome-mediated activation of caspase-1, which processes the precursor into its mature form. In this study, we show that infection with Mycoplasma sp. or treatment with the synthesized Mycoplasma-derived lipoprotein FSL1 elicits the secretion of IL1 beta from monocytes and macrophages at levels, which are comparable to those seen upon infection with known inflammasome activators. Additionally, we provide evidence that Mycoplasma infection stimulates the nuclear to cytosolic redistribution of the inflammasome adaptor protein ASC, an event, which our previous work has shown to be necessary for inflammasome formation. Furthermore, we demonstrate that the IL-1beta released by these cells is the mature, processed form, and that Mycoplasmainduced secretion of IL-1beta is dependent upon the expression of the key inflammasome adaptor protein ASC. 


\section{INTRODUCTION}

Inflammation in response to exogenous and endogenous danger signals is a complex and highly regulated process. Cytokines are soluble mediators that play an indispensable role in the direction and facilitation of the immune system in response to a wide array of pathogenic invaders. Interleukin-1beta (IL-1beta) is a crucial inflammatory cytokine, which functions in the recruitment of white blood cells to the site of invasion, activation of other pro-inflammatory cytokines, and as a potent fever inducer (Dinarello, 1996). Cellular production of mature IL1 beta is a complex process that involves regulation at multiple steps along the way. IL-1 beta is initially produced by the cell as an inactive $33 \mathrm{kDa}$ precursor, which requires cleavage by active caspase- 1 in order to become the fully active 17.5 kDa cytokine released by the cell (Thornberry, 1992). Furthermore, caspase-1 itself must also be activated in order to exhibit proteolytic activity and recent evidence has demonstrated that this is achieved through the assembly of a multi-protein activation platform (Martinon, 2002). These complexes, which have been termed inflammasomes, form in response to a large number of diverse pathogenic associated molecular patterns (PAMPs) as well as endogenous danger associated molecular patterns (DAMPs). Assembly of inflammasome complexes enables activation of cytosolic pattern recognition Nod-like receptors (NLRs) by PAMPs and DAMPs to trigger downstream caspase-1 activation (Franchi L, 2009,Martinon F, 2007,Tschopp, 2003). Thus, essential inflammasome components include NLRs, pro-caspase-1, and a small 
adaptor protein called apoptosis associated speck-like protein containing a CARD domain (ASC). This small adaptor protein consists of an N-terminal Pyrin domain (PYD), and a C-terminal caspase recruitment domain (CARD), which both function to bridge the activation of NLRs to the generation of active caspase-1 (Srinivasula, 2002,Stehlik, 2003,Taniguchi S, 2007,Yamamoto, 2004,Yu, 2006). Inflammasome assembly occurs when a cytosolic NLR protein recognizes a specific pathogenic molecular pattern or endogenous danger signal, which results in the oligomerization of the receptor in an ATP-dependent manner (Duncan JA, 2007,Faustin B, 2007). Furthermore, activation of NLRs enables them to recruit the adaptor protein ASC via homotypic interaction between the PYRIN domains of each protein (Stehlik, 2007). ASC subsequently recruits procaspase-1 via a homotypic CARD-CARD interaction resulting in activation of caspase- 1 by the induced proximity mechanism (Srinivasula, 2002,Stehlik, 2003,Yu HB, 2008).

To date, a number of pathogenic as well as endogenous inflammasome activators have been identified. The NALP3 inflammasome is the currently the best characterized, and has been found to be required for IL-1beta release in response to both pathogenic and endogenous molecular patterns, including bacterial RNA (Kanneganti, 2006), double-stranded viral RNA (Kanneganti TD, 2006) the peptidoglycan component muramyl dipeptide (Kanneganti TD, 2006,Marina-García N, 2008,Martinon, 2004,Pan Q, 2007), uric acid crystals (Martinon, 2006), and extracellular ATP (Mariathasan, 2006). Additionally, 
ligands have also been identified for, Nod1 and Nod2, which respond to and ieDAP and MDP, respectively ((Chamaillard, 2003,Girardin, 2003a,Girardin, 2003b).

Mycoplasma species are unusual pathogens that are poorly characterized. They are the smallest known free-living organisms, which are distinguished from other bacteria by their lack of cell wall. This unique feature of mycoplasmas makes them difficult to culture, and as a result infections with these organisms often go undiagnosed. Furthermore, their lack of cell wall also renders them resistant to many commonly used antimicrobials, which in addition to acquired resistance, makes diseases caused by these organisms difficult to treat (Bébéar $\mathrm{CM}, 2005)$. Although Mycoplasma species have been associated with a large number of organ-specific human diseases including pneumonia, pericarditis, meningitis, and pelvic inflammatory disease, very little is known about their interaction with the host defense system (Baseman JB, 1997,Sacht G, 1998,Waites KB, 2004). Furthermore, these organisms have also been implicated in the pathogenesis of chronic inflammatory diseases such as rheumatoid arthritis, asthma, and Crohn's disease (Sacht G, 1998). Since IL1 beta is a key component of the innate immune response to pathogens, and dysregulation of its release is strongly associated with a number of autoinflammatory disorders, we investigated whether it played a role in the host response to Mycoplasma invasion. 
In this study, we identify Mycoplasma as a novel activator of the inflammasome. We demonstrate that induction of human monocytic cells with both live and heat-killed Mycoplasma species as well as the synthesized lipoprotein FSL1 elicits the secretion of fully mature IL-1beta in an ASCdependent manner. In addition, we show that incubation of THP-1 macrophages results in the nuclear to cytoplasmic redistribution of ASC, as well as the formation of perinuclear aggregates. Our results provide evidence for Mycoplasma-mediated activation of the inflammasome, and indicate that IL-1beta release is a key component in the host defense against these pathogens. 


\section{EXPERIMENTAL PROCEDURES}

\section{Cell culture.}

THP-1 and U-937 cells were obtained from the American Type Culture Collection and maintained as recommended by ATCC. THP-1 cells were used at low passage numbers and were regularly tested for mycoplasma infection (MycoAlert, Lonza). Culture supernatants were assayed and a ratio $>1$ between readings with reagent 1 and reagent 2 indicates the presence of mycoplasma. Generation of stable THP-1 shASC knock-down cells has been previously described (Bryan NB, 2009).

Differentiation of THP-1 cells into macrophages was achieved by seeding the cells onto glass cover slips coated with collagen I $\left(5 \mu \mathrm{g} / \mathrm{cm}^{2}\right.$ and incubating them overnight in culture medium supplemented with $15 \mathrm{ng} / \mathrm{ml}$ of phorbol 12-myristate 13-acetate (PMA) followed by further culture for 2 days. Where indicated, cells were treated with $100 \mathrm{ng} / \mathrm{mL}$ of synthesized FSL1, $2 \times 10^{5} \mathrm{cfu} / \mathrm{ml}$ of heat killed Acholeplasma laidlawii (HKAL), Legionella pneumophila (HKLP), and Staphylococcus aureus (HKSA) (InvivoGen).

\section{Isolation of primary monocytes from human peripheral blood.}

Human monocytes were isolated by Ficoll-Hypaque centrifugation (Sigma) from heparanized blood. Mononuclear cells were removed, washed in Hank's Buffered Salt Solution (HBSS), resuspended in serum-free DMEM and isolated by adherence to plastic dishes. Adherent monocytes were washed and incubated in complete RPMI. 


\section{Immunofluorescence.}

$1 \times 10^{6} \mathrm{THP}-1$ or U-937 cells were fixed in $3.7 \%$ paraformaldehyde / PBS for 30 minutes at room temperature. Next, cells were washed twice with PBS, and incubated with $50 \mathrm{mM}$ glycine/PBS for 5 minutes. They were then blocked and permeabilized in $1 \%$ BSA / $1.5 \%$ goat serum / $0.5 \%$ saponin / PBS for 30 minutes. Following blocking, the cells were incubated with a primary polyclonal anti-ASC antibody from Chemicon (1:200) directed against the CARD domain, or a custom-raised affinity purified polyclonal antibody, which recognizes the linker domain (CS3 1:1000). After washing in $0.5 \%$ saponin / PBS, cells were incubated with an AlexaFluor 488 anti-rabbit secondary antibody in addition to phalloidin 546, which stains actin, and ToPro-3, which stains DNA, in the blocking solution. Finally, cells were washed in PBS containing $0.5 \%$ saponin, followed by PBS alone, and mounted with Fluoromount. Suspension cells were stained as described above, and then adhered to poly-L-lysine coated slides using cytocentrifugation. Images were acquired by laser scanning confocal microscopy using a ZEIS LSM 510 microscope under a 100x oil immersion objective.

\section{Subcellular fractionation.}

$10^{6}$ cells were resuspended in nuclear extraction buffer (10 mM HEPES pH 8.0, 0.5\% NP40, 1.5mM MgCl2, $10 \mathrm{mM} \mathrm{KCl,} 0.5 \mathrm{mM} \mathrm{DTT}$, and $200 \mathrm{mM}$ sucrose, supplemented with protease and phosphatase inhibitors). After a 5 minute incubation on ice, nuclei were pelleted by centrifugation at $14,000 \mathrm{rpm}$ for 15 seconds, and the cytosolic 
supernatant was transferred to a new tube. Nuclei were washed once with $1 \mathrm{ml}$ of extraction buffer, and lysed in RIPA buffer (1\% NP40, 1\% deoxycholic acid, $0.1 \%$ SDS, $0.15 \mathrm{M} \mathrm{NaCl}, 0.1 \mathrm{M}$ sodium phosphate $\mathrm{pH} 7.4,2$ 9mM EDTA supplemented with protease and phosphatase inhibitors). Salt and detergent concentrations of the cytosolic fraction were adjusted to RIPA buffer concentrations and all fractions were fully solubilized by brief sonication. $50 \mu \mathrm{g}$ of protein lysates were separated by SDSPAGE, transferred to a PVDF membrane, and probed with a monoclonal anti-ASC antibody at a 1:1000 dilution (MBL), an HRP-conjugated secondary antibodies at a 1:5000 dilution (Amersham Pharmacia) in conjunction with an ECL detection system (Pierce). Membranes were stripped and re-probed with anti-GAPDH at a 1:1000 dilution (Sigma) and anti-Lamin A at a 1:500 dilution (Santa Cruz Biotechnology) antibodies as controls for cytosolic and nuclear fractions, respectively.

\section{Measurement of secreted IL-1beta .}

IL-1 beta secretion was quantified in culture supernatants using a commercial ELISA $(B D$

Biosciences). Samples were analyzed in triplicate and each experiment was repeated a minimum of three times. Briefly, THP-1 cells were incubated with HKAL, HKLP, or HKSA in 12-well dishes for 16 hours. Following treatment, the supernatants were centrifuged at $13,000 \mathrm{rpm}$ for 15 minutes in order to remove any residual cells. The cleared supernatants were then either stored at $-80^{\circ} \mathrm{C}$ or immediately analyzed by ELISA. 


\section{Analysis of IL-1beta processing}

Processing of IL-1beta was evaluated in uninduced THP-1 cells and in THP-1 cells incubated with HKAL. Cells were seeded in a 6-well plate and cultured for 24 hours in the presence or absence of HKAL. Following the stimulation, supernatants were removed, and centrifuged at 13,000 rpm for 15 minutes in order to remove any residual cells. The supernatant was then transferred to a fresh tube, and concentrated using a Nanosep centrifugal device containing a 3K omega membrane (Sigma). Upon removal of the supernatants, the cells were lysed in RIPA buffer (see above) and assayed for protein concentration using BCA protein quantification kit (Pierce). $75 \mu \mathrm{g}$ of the protein lysates, and the concentrated supernatants were supplemented with Laemli's loading buffer, and analyzed by SDS-PAGE on a $12 \%$ acrylamide gel. The proteins were then transferred to a PVDF membrane, and probed with an anti-IL-1 beta antibody (1:1000) (Millipore), which recognizes both the unprocessed 33kDa form and the processed $17 \mathrm{kDa}$ form followed by HRP-conjugated secondary antibody. The proteins were subsequently detected using an ECL system (Pierce). 
RESULTS

\section{Mycoplasma species stimulate the release of mature IL-1beta from human} monocytes.

Secretion of mature IL-1beta is a two-step process that involves transcriptional upregulation of the precursor form by NF-kB, which is subsequently processed upon inflammasome-mediated caspase-1 activation. Therefore, we first determined if heat-killed Acholeplasma laidlawii (HKAL) were capable of stimulating the release of IL-1beta from human monocytes. Culture supernatants from primary monocytes (Fig. 1A) as well as from the human monocytic THP-1 cell line (Fig. 1B) were analyzed by ELISA following incubation with HKAL. For both primary human monocytes and THP-1 cells, IL-1beta secretion was greatly enhanced in cells incubated with HKAL over the untreated control cells. In addition, the amount of IL1 beta released by THP-1 cells in response to HKAL treatment was comparable to the amount elicited by the known inflammasome activators Legionella pneumophilia (HKLP) and Staphylococcus aureus (HKSA). In order to confirm that the IL-1beta secreted in response to HKAL was the mature $17.5 \mathrm{kD}$ form, we concentrated the culture supernatant of THP-1 cells following incubation with HKAL and analyzed it by western blot for IL-1beta. While both the $33 \mathrm{kD}$ precursor, and the mature $17 \mathrm{kD}$ form were observed in cell lysates, only the processed form was detected in the supernatant treated cells (Fig 1C). Furthermore, while untreated control cells displayed very low levels of the IL-1beta precursor in the cell lysate, it was not 
observed in the supernatant, and the active form was not detected at all. Next, we evaluated whether infection with live Mycoplasma sp. could stimulate the release of IL-1beta from human monocytes. Culture supernatants fromTHP-1 cells were analyzed as for the presence of secreted IL-1beta as above following infection with live Mycoplasma (Fig. 1D). We found that live infection with Mycoplasma species did result in an elevation in the release of IL-1beta over uninfected control cells. Active, live infection was confirmed using the MycoAlert assay system (Fig. 1E).

\section{Mycoplasma infection induces the nuclear to cytoplasmic redistribution of the inflammasome adaptor ASC.}

The release of mature $17 \mathrm{kDa}$ IL-1 beta requires the activation of caspase- 1 through inflammasome assembly. Therefore, we investigated the role of the central inflammasome adaptor, ASC, in Mycoplasma-induced IL-1beta release. Our recent work demonstrated that inflammasome activation and subsequent IL-1 beta secretion requires the translocation of ASC from the nucleus to the cytoplasm (Bryan NB, 2009). Therefore, we used immunofluorescence to evaluate the subcellular localization of endogenous ASC in the human monocytic THP-1 and U-937 cell lines

following infection with Mycoplasma. In agreement with our previous report, ASC was localized almost exclusively in the nucleus in resting monocytes (Fig. 2A). However, live Mycoplasma infection resulted in a completely cytosolic distribution of ASC (Fig. 2B). 


\section{Stimulation of THP-1macrophages with HKAL induces the formation of}

cytosolic ASC aggregates.

Our previous study revealed that in differentiated macrophages, ASC not only relocalized to the cytoplasm, but it also formed perinuclear aggregates reminiscent of those characterized in overexpression studies. Thus, we next evaluated how stimulation with HKAL impacted ASC localization in THP-1 macrophages. Similar to the known inflammasome activators HKSA and HKLP, HKAL also induced the formation of perinuclear ASC aggregates in THP-1 macrophages (Fig 3A). To confirm our immunofluorescence results, we fractionated proteins from resting or Mycoplasma-infected THP-1 and U-937 cells into nuclear and cytosolic components, and used immunoblotting to determine where ASC was localized. Fractionation efficiency was confirmed by stripping and re-probing for cytosolic GAPDH and nuclear Lamin A proteins. While a substantial portion of ASC was found in the nuclear fraction of resting THP-1 and U-937 cells, active Mycoplasma infection resulted in the exclusive cytoplasmic localization of ASC (Fig. 3B). Based upon these results, and those in Figure 2, we conclude that Mycoplasma induces nuclear to cytosolic redistribution as well as perinuclear aggregate formation of endogenous ASC.

\section{ASC is required for Mycoplasma-induced IL-1beta release.}

In order to firmly link inflammasome activation as an essential component of Mycoplasma-mediated IL-1 beta release, we generated THP-1 cells, which stably 
expressed plasmids encoding shRNA for either the inflammasome adaptor ASC, or firefly luciferase as a control. Using these cells, we examined whether ASC expression was necessary for the release of IL-1beta in response to activation with HKAL. Culture supernatants from sh-ASC or sh-control THP-1 cells were analyzed by ELISA for secreted IL-1 beta under resting conditions or following induction with HKAL, HKLP, or HKSA. While a large induction in IL-1beta secretion was observed in response to incubation of HKAL, HKLP, or HKSA with the control cells, loss of ASC resulted in a significant inhibition of IL-1beta release following activation with all three pathogens (Fig. 4). Thus, the inflammasome adaptor ASC is essential for the secretion of IL-1beta in response to stimulation with HKAL.

\section{The mycoplasma lipoprotein FSL1 is sufficient to cause macrophage}

\section{inflammasome activation.}

Inflammasome activation occurs upon recognition of specific pathogen associated molecular patterns (PAMPs) by cytoplasmic NLRs, which function as pattern recognition receptors. Therefore, we next identified a specific which Mycoplasma-derived PAMP, which could induce IL-1beta processing. Using the THP-1 cells stably transfected with shRNA, we examined the ability of the purified Mycoplasma lipoprotein FSL1 to induce IL-1beta maturation and release. Treatment with synthesized FSL1 (Invivogen) stimulated the release of IL-1beta as efficiently as heat-killed A. laidlawii in an ASC-dependent manner (Fig. 5a). Furthermore, we once again that the released IL-1beta was indeed the 
processed, mature form by analyzing cell lysates and supernatants from activated cells by immunoblotting (Fig. 5b). These data suggest that FSL1 is capable of activating the inflammasome and thus is one of the NLR-recognized mycoplasma-derived PAMPs. 


\section{DISCUSSION}

In spite of their involvement in a large number of human diseases, the interaction between these organisms and the host immune system has not been fully characterized. Mycoplasma sp. are completely reliant upon host cells for nutrition, and therefore do not induce cell death. As a result, the presence of these organisms frequently goes undetected. One mechanism by which they do initiate an immune response is through the stimulation of macrophages via lipoproteins containing a diacylated cysteine residue. The macrophage response is mediated by TLR2/TLR6 heterodimers, which induce the downstream activation of NF-kB through the adaptor MyD88 (Garcia J, 1998,Nishiguchi M, 2001,Okusawa T, 2004,Sacht G, 1998,Takeuchi O, 2000). In this study, we examined the innate immune response to infection with Mycoplasma sp., and provide evidence that they are capable of activating the inflammasome, and eliciting release of mature IL-1beta.

We determined that incubation of both primary macrophages as well as THP1 cells with heat-killed A. laidlawii, induced the secretion of IL-1beta (Fig. 1a and b). Furthermore, the amount of IL-1 beta release elicited by incubation with $A$. laidlawii was comparable to the amount released in response to the known inflammasome activators HKSA (NLRP3), and HKLP (NLRP3 and NLRC4) (Case CL, 2009,Mariathasan, 2006,Vinzing M, 2008). Additionally, live infection of THP-1 monocytes with Mycoplasma sp. also resulted in elevated release of IL1 beta (Fig. 1d and e). 
Generation of mature IL-1 beta is widely believed to be a two-step process that involves the initial transcriptional upregulation of the precursor by NF-kB followed by inflammasome assembly and caspase- 1 activation. NF-kB activation in monocytic cells is usually achieved through stimulation of Toll-like receptors (TLRs), while inflammasome assembly requires the activation of cytoplasmic NLRs ((Becker CE, 2007,Mariathasan, 2007). Mycoplasma sp. have previously been shown to interact with TLR2/TLR6 and thereby initiate downstream activation of NF-kB, which regulates the transcription of pro-IL-1beta. Therefore, we confirmed that the II-1 beta secreted from cells was the fully active, mature form, which is produced as a result of caspase- 1 activation by analyzing the concentrated supernatant as well as the cell lysates by immunoblotting. The vast majority of IL-1 beta present in the cell lysate and all of the IL-1beta present in the supernatant of Mycoplasma-activated cells was the mature $17.5 \mathrm{kD}$ form (Fig. 1c). This data demonstrates that the IL-1beta detected by ELISA is indeed the processed, mature form. Furthermore, the presence of the mature $17.5 \mathrm{kD}$ form of IL-1 beta in the cell lysate indicates that it is being processed by caspase-1 prior to its release from the cell, and not by extracellular proteases.

Next, we evaluated how Mycoplasma infection impacted the subcellular distribution of the inflammasome adaptor protein ASC. ASC is a crucial mediator of inflammasome assembly initiated by the activation of multiple NLRs following recognition of their respective PAMPs or DAMPs. We recently demonstrated that in resting monocytes and macrophages, ASC is localized to the nucleus. 
However, upon stimulation with previously identified inflammasome ligands, ASC is rapidly redistributed to the cytoplasm, where it subsequently forms large perinuclear aggregates that incorporate the key components of the inflammasome. Furthermore, we showed that nuclear export of ASC is essential to inflammasome assembly and IL-1beta maturation (Bryan NB, 2009). Live infection of both THP-1 and U-937 monocytes with Mycoplasma sp. resulted in the complete cytoplasmic redistribution of ASC, while the uninfected cells retained it inside the nucleus. (Fig. 2a and b and 3b).

We then examined whether stimulation of THP-1 macrophages with Mycoplasma could induce the formation of perinuclear ASC aggregates. Just as observed with the known inflammasome activators HKLP and HKSA, incubation of THP-1 macrophages with HKAL induced the formation of ASC aggregates. This finding provides further evidence that Mycoplasma sp. induce inflammasome assembly based upon our previous work demonstrating that ASC co-localizes with other key inflammasome proteins including caspase-1 and NLRP3 receptor in these structures (Bryan NB, 2009).

Next, we established that ASC is required for IL-1beta release in response to activation with HKAL. To achieve this, we employed THP-1 cells with a stable knock-down of endogenous ASC (Fig. 4b). These cells exhibited a greatly diminished capacity to secrete IL-1beta following induction with HKAL, HKSA, or HKLP in comparison to the control cells (Fig. 4). Therefore, release of IL-1beta in response to HKAL is dependent upon ASC. 
Finally, we identified the lipoprotein FSL1 as an inflammasome-activating PAMP. We demonstrated that treatment of THP-1 cells with purified FSL1 elicited the secretion of mature IL-1beta in an ASC-dependent manner. Furthermore, the levels of IL-1beta released were comparable to those observed following induction with HKAL.

In summary, we demonstrated that infection with Mycoplasma sp. causes the nuclear to cytoplasmic redistribution of the inflammasome adaptor protein ASC and elicits the secretion of mature IL-1beta in an ASC-dependent manner. Our data identify Mycoplasma sp. as a novel activator of the inflammasome with the lipoprotein FLS1 acting as one of the NLR-activating PAMPs, providing further insight into the host defense mechanisms employed against this pathogen. Additionally, our results also suggest the possibility that chronic inflammasome activation and subsequent IL-1beta release may be the mechanism by which latent Mycoplasma infection can lead to the development of chronic inflammatory disorders such as asthma and rheumatoid arthritis. While the specific NLR receptors necessary for the recognition of Mycoplasma sp. remain elusive, the requirement for ASC indicates that one or more of the fourteen receptors, which contain a PYD are involved. Studies aimed at identifying the receptors involved in the response to Mycoplasma sp. are currently underway. 


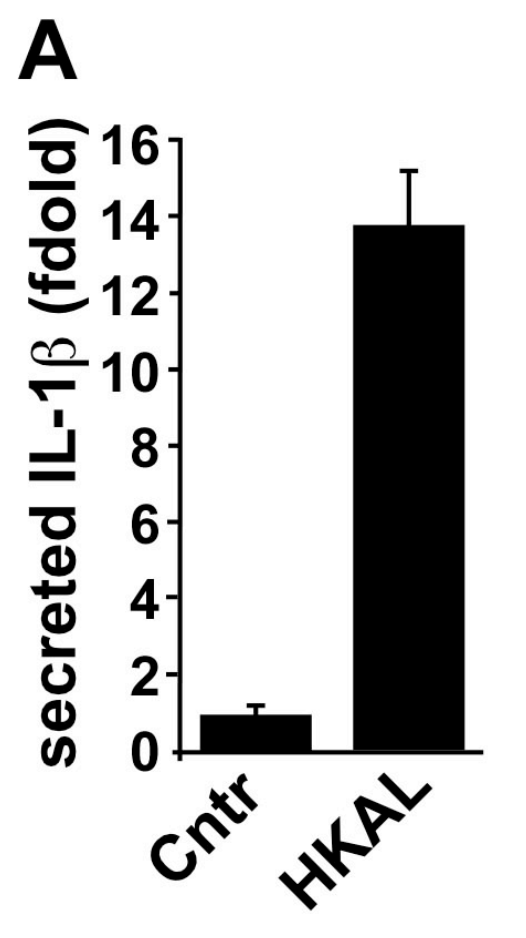

B

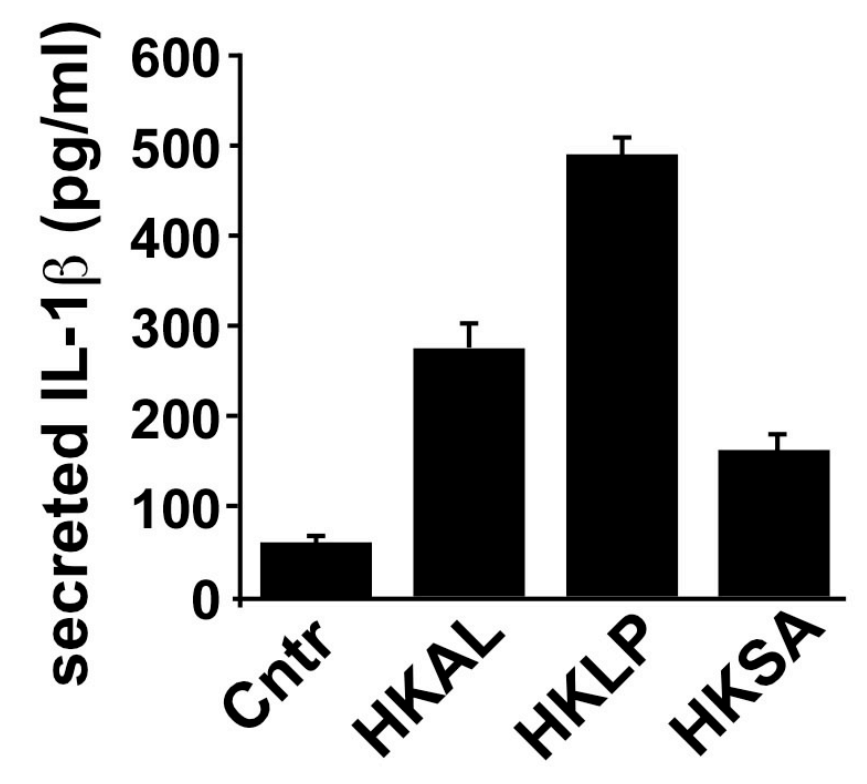

Figure 1. Mycoplasma sp. Induce secretion of mature IL-1beta from THP-1 monocytes.

(A). Culture supernatants from primary human macrophages under resting conditions or stimulated with heat-killed Acholeplasma laidlawii (HKAL) were collected and analyzed for secretion of IL-1beta by ELISA. Results are presented as a fold - increase in secretion over the untreated control.

(B). Culture supernatants from THP-1 monocytes under resting conditions, or following treatment with HKAL $\left(2 \times 10^{5}\right)$, Gram-negative HKLP $\left(2 \times 10^{5} \mathrm{cfu}\right)$, or Gram-positiveHKSA $\left(2 \times 10^{5} \mathrm{cfu}\right)$ were collected and analyzed for the presence of secreted IL-1 beta. Results represent three independent experiments +/- SD. and are presented as $\mathrm{pg} / \mathrm{mL}$ of released IL-1beta. 

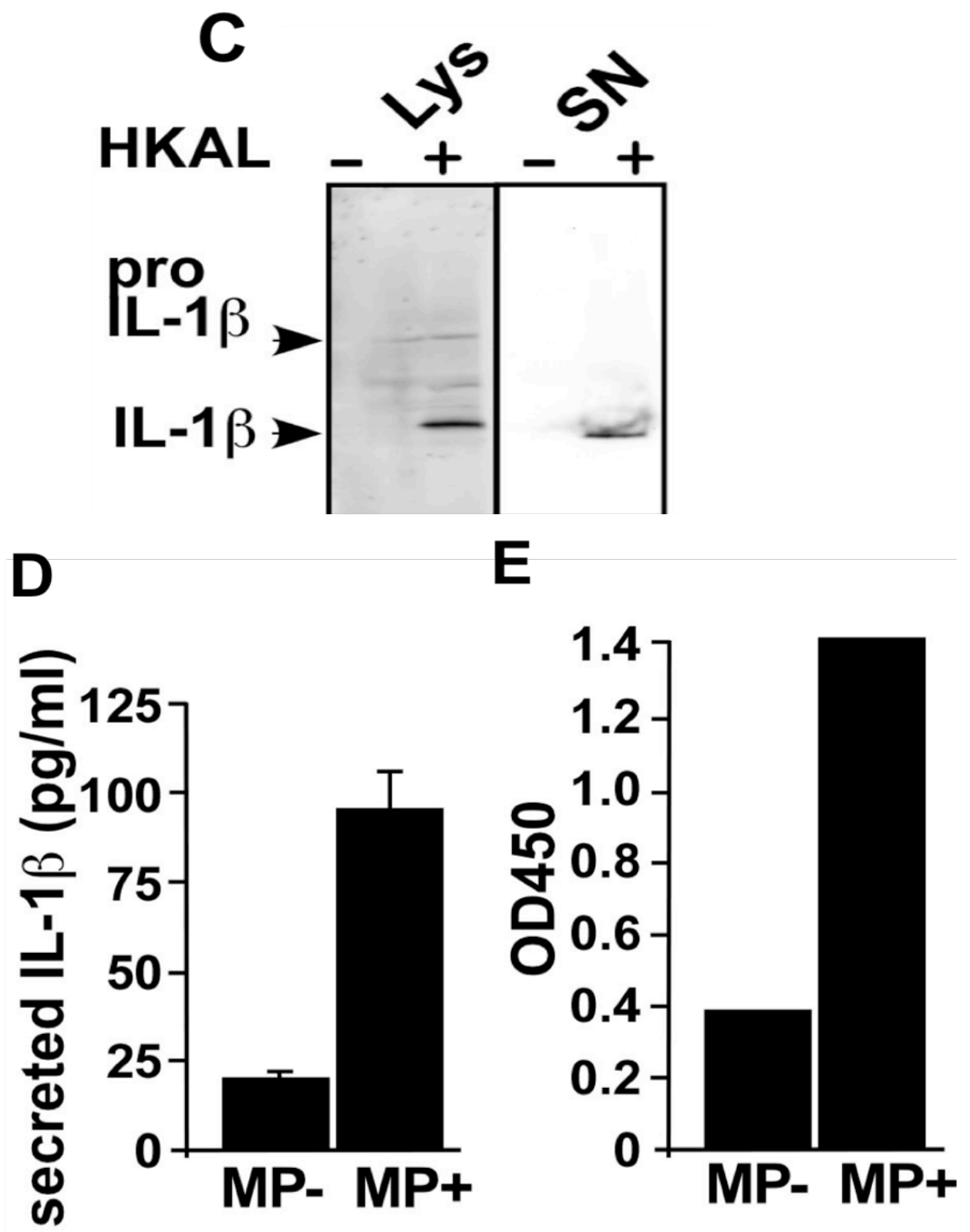

(C). THP-1 cells were either left untreated or stimulated with HKAL. The culture supernatants and cell lysates were collected separately, and analyzed by immunoblot for the expression of processed and unprocessed IL-1beta.

(D). Culture supernatants were collected from THP-1 cells under resting conditions or following incubation with a live Mycoplasma sp. and analyzed for released IL-1 beta by ELISA. Results represent an average of three independent experiments +/- SD, and are presented as $\mathrm{pg} / \mathrm{mL}$ of secreted IL-1beta. The infection status of the cells was confirmed by evaluation of culture supernatants using a commercial kit (E). 

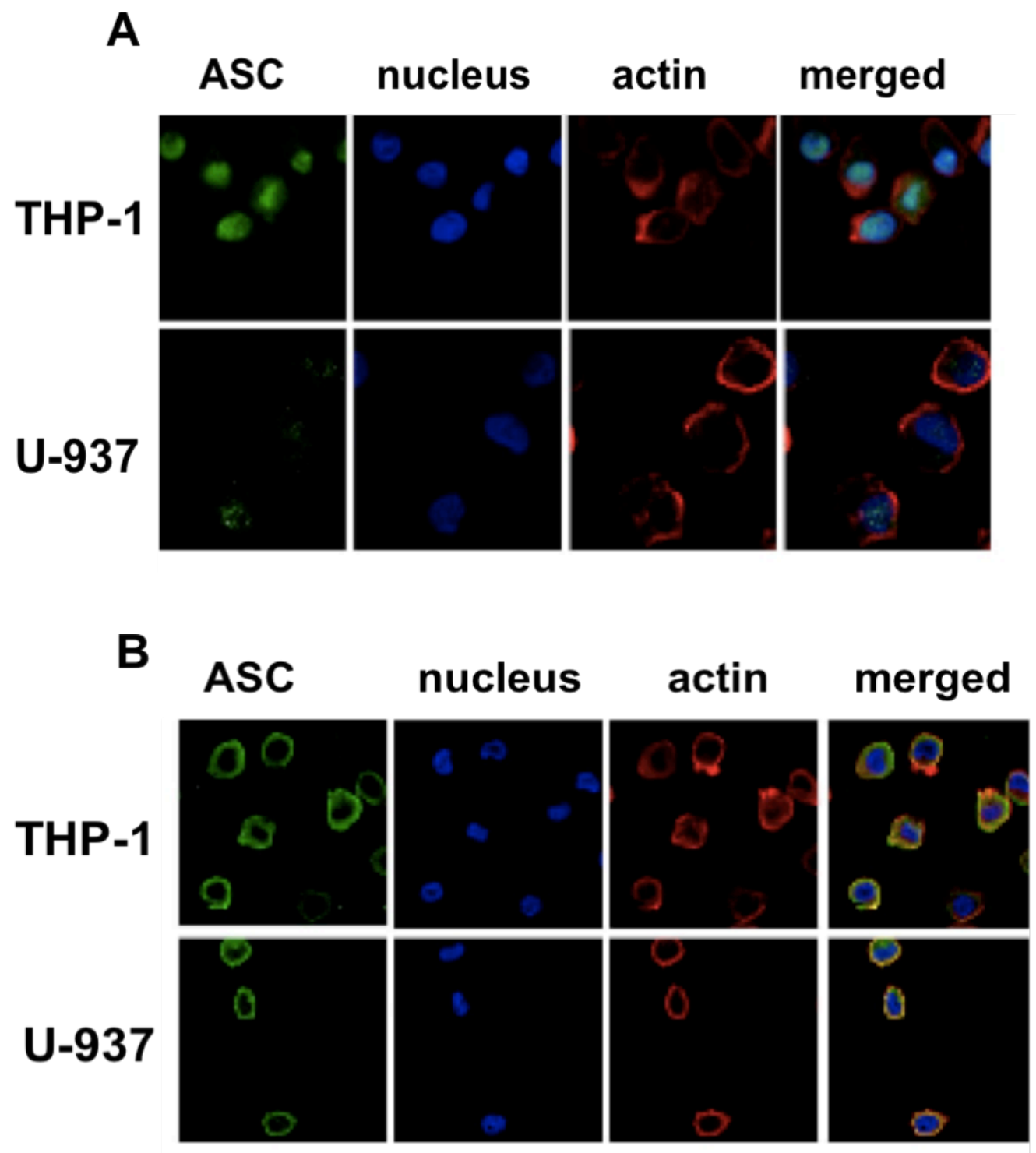

Figure 2. Live infection with Mycoplasma sp. induces nuclear to cytosolic redistribution of ASC in human monocytic cell lines.

Subcellular localization of endogenous ASC was evaluated in two human monocytic cell lines, THP-1 and U-937 under resting (A), and upon live infection with Mycoplasma sp. (B). Cells were fixed and immunostained with a monoclonal antibody against ASC (MBL). An AlexaFluor-488-conjugated secondary antibody was used in conjunction with ToPro-3 to counterstain the nuclear DNA, and AlexaFluor 546-conjugated phalloidin to visualize the actin cytoskeleton. Images were obtained using laser scanning confocal microscopy and the panels from left to right represent ASC (green), nucleus (blue), actin (red), and a merged compilation. 


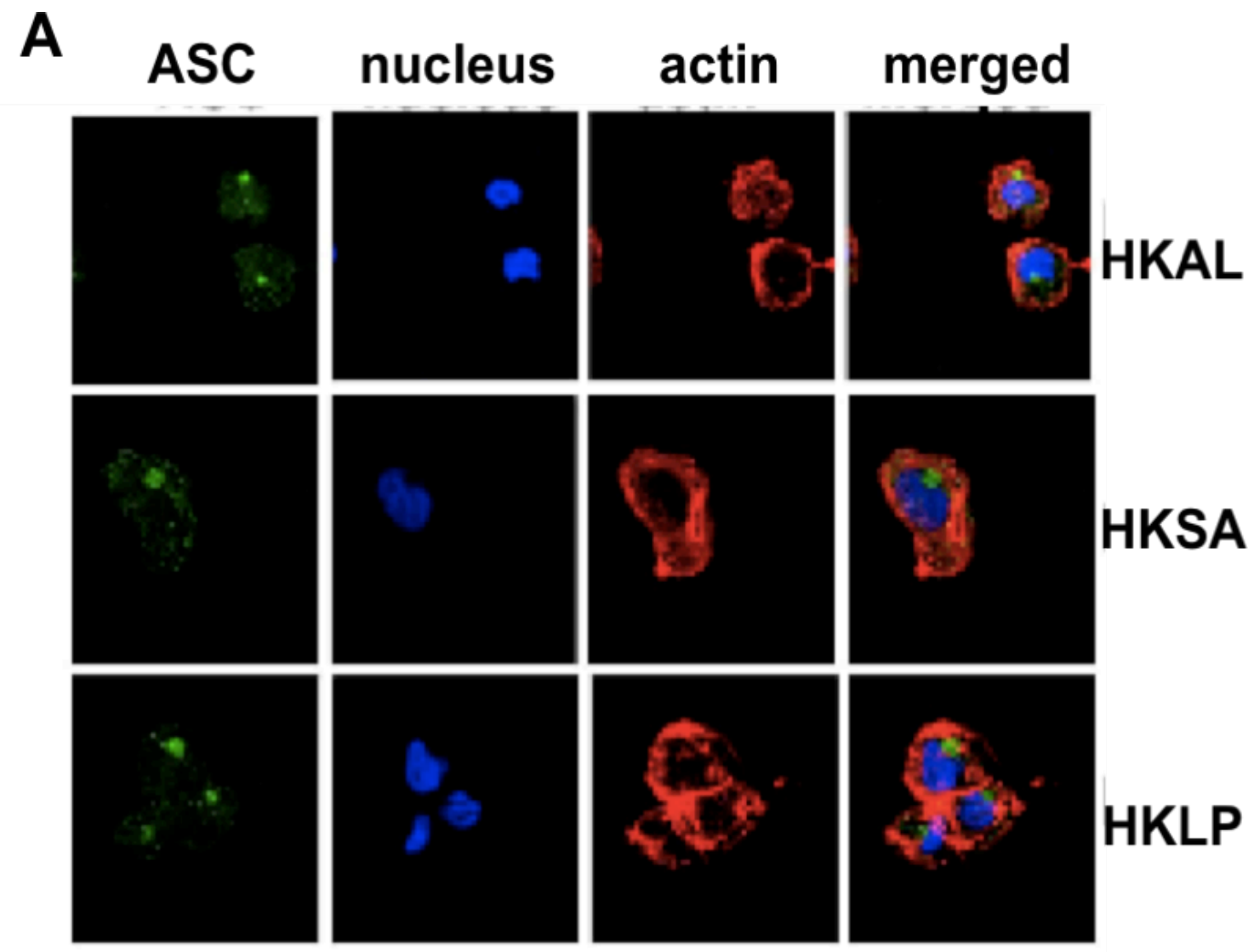

Figure 3. Stimulation of THP-1 macrophages with HKAL results in formation of perinuclear ASC aggregates.

(A). Subcellular localization of endogenous ASC was evaluated in PMAdifferentiated THP-1 macrophages following activation with HKAL, Gram-positive HKSA, and Gram-negative HKLP. Cells were fixed following 6 hours of incubation and immunostained with a custom-raised polyclonal anti-ASC antibody (CS3). An anti-rabbit AlexaFluor-488 conjugated secondary antibody was used in addition to ToPro-3 to counterstain the nuclear DNA, and AlexaFluor-546 conjugated phalloidin in order to visualize the actin cytoskeleton. Images were acquired using laser scanning confocal microscopy (Zeiss LSM 510)and the panels from left to right demonstrate the distribution of ASC (green), nuclei (blue), the actin cytoskeleton (red) and a merged compilation. 
B

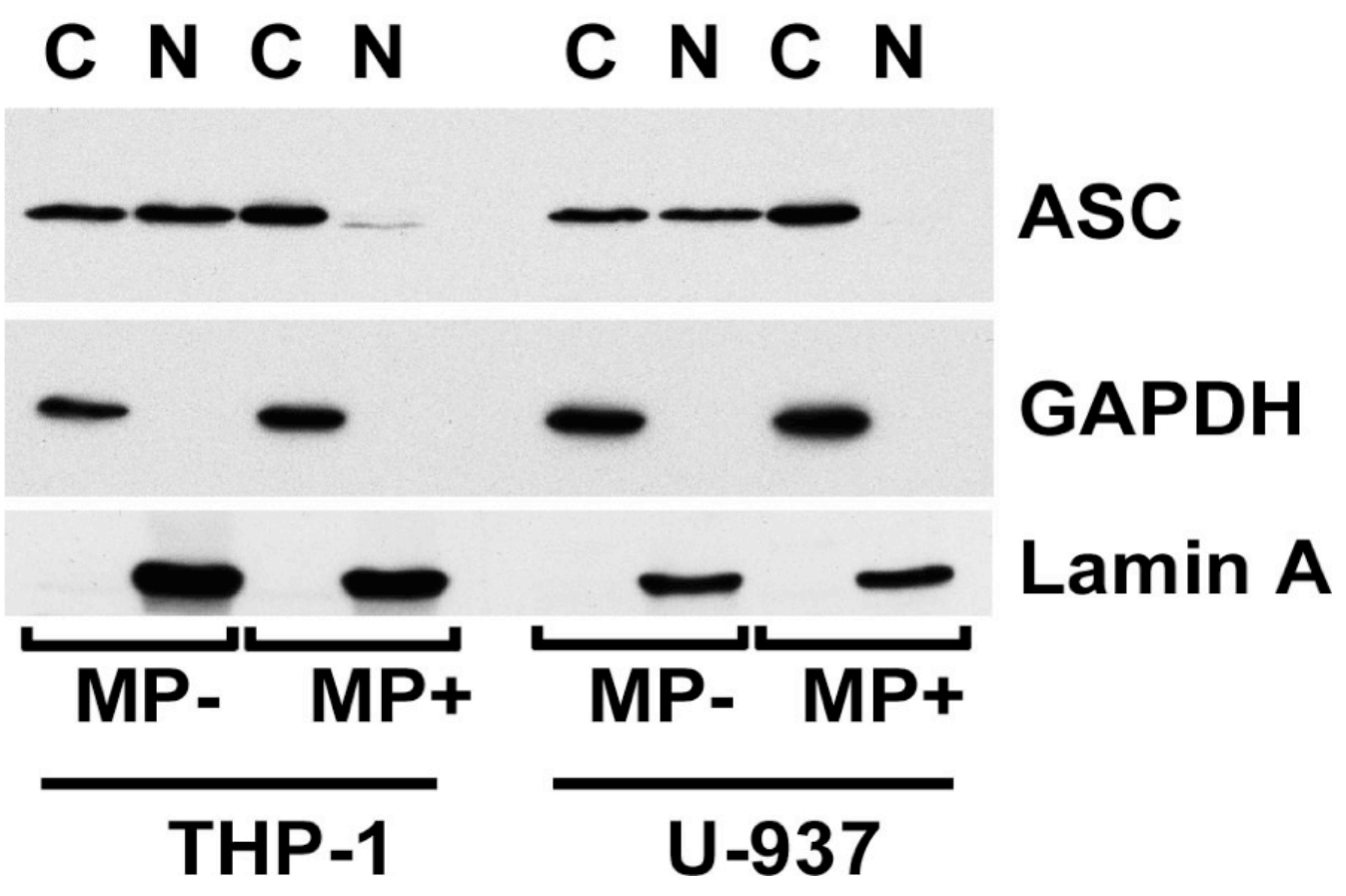

(B). Localization of ASC was analyzed by subcellular fractionation of THP-1 and U-937 monocytes under resting conditions and following incubation with live Mycoplasma sp. $1 \times 10^{6}$ cells were fractionated by differential centrifugation into nuclear and cytoplasmic fractions, and normalized for protein contents. 50ug of protein lysate was analyzed by immunoblotting with a monoclonal anti-ASC antibody, followed by an anti-mouse HRP-conjugated secondary antibody. Fractionation efficiency was confirmed by stripping the membrane, and reprobing for cytosolic GAPDH and nuclear Lamin A proteins. 


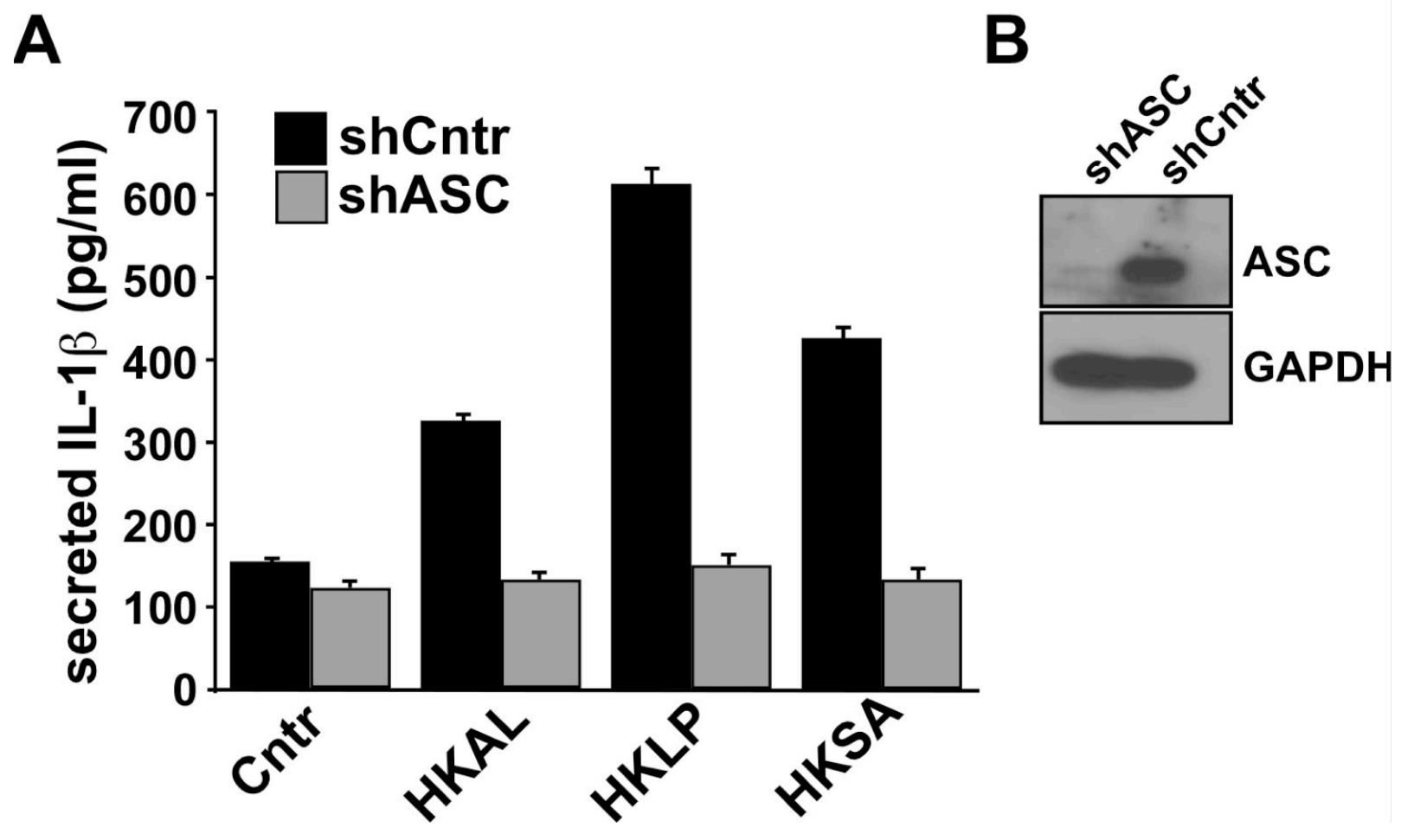

Figure 4. ASC is required for IL-1beta secretion induced by HKAL.

(A). THP-1 cells were stably transfected with either control shRNA (black bars) or shRNA targeting ASC (gray bars). $1 \times 10^{6}$ cells were seeded in triplicate into a 12-well dish and were either left untreated or were stimulated with HKAL $\left(1 \times 10^{5}\right.$ cfu), HKLP $\left(1 \times 10^{5} \mathrm{cfu}\right)$, or HKSA $\left(1 \times 10^{5} \mathrm{cfu}\right)$. Supernatants were then collected and analyzed for secreted IL-1 beta by ELISA. Results represent an average of three independent experiments $+/-\mathrm{SD}$ and are presented $\mathrm{as} \mathrm{pg} / \mathrm{mL}$ of secreted IL-1beta.

(B). Efficient knock-down of ASC was confirmed in stably transfected THP-1 cells by immunoblot. $1 \times 10^{6}$ cells stably expressing control shRNA and ASC shRNA were directly lysed in Laemli buffer and separated by SDS-PAGE. The proteins were then transferred to PVDF membrane, which was probed with a custom-raised polyclonal anti-ASC antibody (CS3) followed by an anti-rabbit HRP-conjugated secondary antibody. To ensure equal loading, the blot was subsequently stripped and re-probed with a monoclonal anti-GAPDH antibody. 

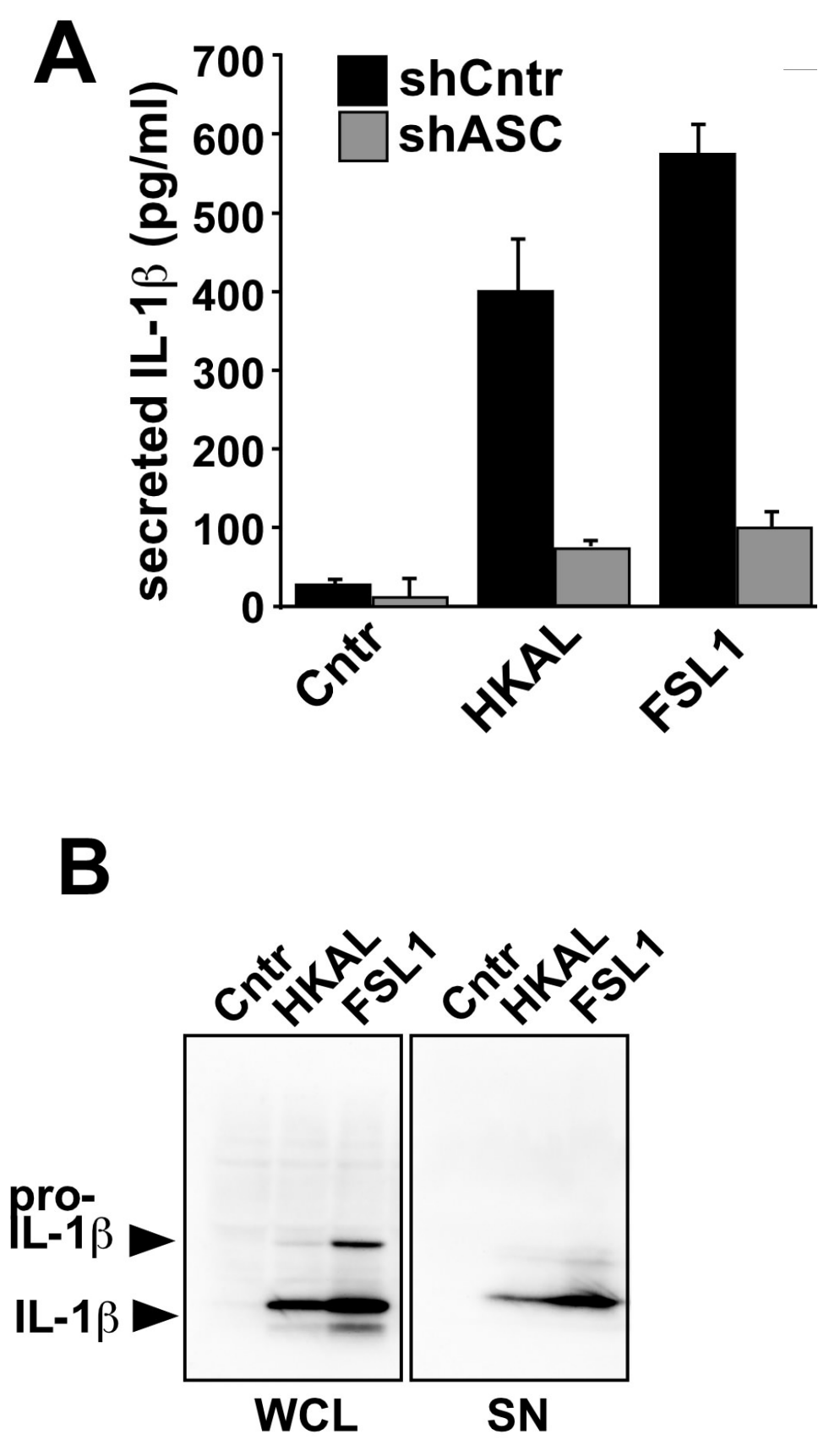

Figure 5. The mycoplasma lipoprotein FSL1 is sufficient to cause macrophage inflammasome activation.

THP-1 cells and THP-1 cells with ASC knock-down were left untreated, treated with HKAL $\left(2 \times 10^{5} \mathrm{cfu} / \mathrm{ml}\right)$, or purified FSL1 $(100 \mathrm{ng} / \mathrm{ml})$, followed by analysis of culture supernatants for released IL-1 $\beta$ by ELISA $(A)$ and upon concentration of the culture supernatant from THP-1 cells also by immunoblot in culture supernatants $(\mathrm{SN})$ and whole cell lysates $(\mathrm{WCL})(\mathrm{B})$. 


\section{REFERENCES}

Baseman JB, T.J. (1997) Mycoplasmas: sophisticated, reemerging, and burdened by their notoriety. Emerg Infect Dis, 3:21-32.

Bébéar CM, P.S. (2005) Mechanisms of drug resistance in Mycoplasma pneumoniae. Curr Drug Targets Infect Disord, 5:263-271.

Becker CE, O.N.L. (2007) Inflammasomes in inflammatory disorders: the role of TLRs and their interactions with NLRs. Semin Immunopathol, 29:239-248.

Bryan NB, D.A., Rojanasakul Y, Stehlik C. (2009) Activation of inflammasomes requires intracellular redistribution of the apoptotic speck-like protein containing a caspase recruitment domain. J Immunol, 182:3173-3182.

Case CL, S.S., Roy CR. (2009) Asc and Ipaf Inflammasomes direct distinct pathways for caspase-1 activation in response to Legionella pneumophila. Infection and Immunity, 77:1981-1991.

Chamaillard, M., Hashimoto, M., Horie, Y., Masumoto, J., Qiu, S., Saab, L., Ogura, Y., Kawasaki, A., Fukase, K., Kusumoto, S., Valvano, M.A., Foster, S.J., Mak, T.W., Nunez, G., and Inohara, N. (2003) An essential role for NOD1 in host recognition of bacterial peptidoglycan containing diaminopimelic acid. Nat. Immunol., 4:702-707.

Dinarello, C.A. (1996) Biologic basis for interleukin-1 in disease. Blood, 87:20952147.

Duncan JA, B.D., Wang Y, Willingham SB, Ye Z, Zimmermann AG, Ting JP. (2007) Cryopyrin/NALP3 binds ATP/dATP, is an ATPase, and requires ATP binding to mediate inflammatory signaling. Proc Natl Acad Sci U S A, 104:8041-8046.

Faustin B, L.L., Bruey JM, Luciano F, Sergienko E, Bailly-Maitre B, Volkmann N, Hanein D, Rouiller I, Reed JC. (2007) Reconstituted NALP1 inflammasome reveals two-step mechanism of caspase-1 activation. Molecular Cell, 25:713-724.

Franchi L, E.T., Muñoz-Planillo R, Nuñez G. (2009) The inflammasome: a caspase-1-activation platform that regulates immune responses and disease pathogenesis. Nat Immunol, 10:247-247. 
Garcia J, L.B., Roman-Roman S, Rawadi G. (1998) A Mycoplasma fermentansderived synthetic lipopeptide induces AP-1 and NF-kappaB activity and cytokine secretion in macrophages via the activation of mitogen-activated protein kinase pathways. J Biol Chem, 273:34391-34398.

Girardin, S.E., Boneca, I.G., Carneiro, L.A., Antignac, A., Jehanno, M., Viala, J., Tedin, K., Taha, M.K., Labigne, A., Zahringer, U., Coyle, A.J., DiStefano, P.S., Bertin, J., Sansonetti, P.J., and Philpott, D.J. (2003a) Nod1 detects a unique muropeptide from gram-negative bacterial peptidoglycan. Science, 300:1584-1587.

Girardin, S.E., Boneca, I.G., Viala, J., Chamaillard, M., Labigne, A., Thomas, G., Philpott, D.J., and Sansonetti, P.J. (2003b) Nod2 is a general sensor of peptidoglycan through muramyl dipeptide (MDP) detection. J. Biol. Chem., 278:8869-8872.

Kanneganti, T.D., Ozoren, N., Body-Malapel, M., Amer, A., Park, J.H., Franchi, L., Whitfield, J., Barchet, W., Colonna, M., Vandenabeele, P., Bertin, J., Coyle, A., Grant, E.P., Akira, S., and Nunez, G. (2006) Bacterial RNA and small antiviral compounds activate caspase-1 through cryopyrin/Nalp3. Nature, 440:232-236.

Kanneganti TD, B.-M.M., Amer A, Park JH, Whitfield J, Franchi L, Taraporewala ZF, Miller D, Patton JT, Inohara N, Núñez G. (2006) Critical role for Cryopyrin/Nalp3 in activation of caspase-1 in response to viral infection and double-stranded RNA. J Biol Chem, 281:36560-36568.

Mariathasan, S., Weiss, D.S., Newton, K., McBride, J., O'Rourke, K., RooseGirma, M., Lee, W.P., Weinrauch, Y., Monack, D.M., and Dixit, V.M. (2006) Cryopyrin activates the inflammasome in response to toxins and ATP. Nature, 440:228-232.

Mariathasan, S., and D. M. Monack. (2007) Inflammasome adaptors and sensors: intracellular regulators of infection and inflammation. Nat Rev Immunol:31-40.

Marina-García N, F.L., Kim YG, Miller D, McDonald C, Boons GJ, Núñez G. (2008) Pannexin-1-mediated intracellular delivery of muramyl dipeptide induces caspase-1 activation via cryopyrin/NLRP3 independently of Nod2. Journal of Immunology, 180:4050-4057.

Martinon, F., Burns, K., and Tschopp, J. (2002) The Inflammasome: A molecular platform triggering activation of inflammatory caspases and processing of prolL-b. Mol. Cell, 10:417-426. 
Martinon, F., Agostini, L., Meylan, E., and Tschopp, J. (2004) Identification of bacterial muramyl dipeptide as activator of the NALP3/cryopyrin inflammasome. Curr Biol, 14:1929-1934.

Martinon, F., Petrilli, V., Mayor, A., Tardivel, A., and Tschopp, J. (2006) Goutassociated uric acid crystals activate the NALP3 inflammasome. Nature, 440:237-241.

Martinon F, T.J. (2007) Inflammatory caspases and inflammasomes: master switches of inflammation. Cell Death \& Differ, 14:10-22.

Nishiguchi M, M.M., Takao T, Hoshino M, Shimonishi Y, Tsuji S, Begum NA, Takeuchi O, Akira S, Toyoshima K, Seya T. (2001) Mycoplasma fermentans lipoprotein M161Ag-induced cell activation is mediated by Tolllike receptor 2: role of $\mathrm{N}$-terminal hydrophobic portion in its multiple functions. J Immunol, 166:2610-2616.

Okusawa T, F.M., Nakamura J, Into T, Yasuda M, Yoshimura A, Hara Y, Hasebe A, Golenbock DT, Morita M, Kuroki Y, Ogawa T, Shibata K. (2004) Relationship between structures and biological activities of mycoplasmal diacylated lipopeptides and their recognition by toll-like receptors 2 and 6 . Infection and Immunity, 72:1657-1665.

Pan Q, M.J., Fearns C, Kravchenko VV, Da Silva Correia J, Hoffman HM, Kobayashi KS, Bertin J, Grant EP, Coyle AJ, Sutterwala FS, Ogura Y, Flavell RA, Ulevitch RJ. (2007) MDP-induced interleukin-1beta processing requires Nod2 and CIAS1/NALP3. J Leukoc Biol, 82:177-183.

Sacht G, M.A., Deiters U, Süssmuth R, Jung G, Wingender E, Mühlradt PF. (1998) Activation of nuclear factor-kappaB in macrophages by mycoplasmal lipopeptides. Eur J Immunol, 28:4207-4212.

Srinivasula, S.M., Poyet, J.-L., Razmara, M., Datta, P., Zhang, Z., and Alnemri, E.S. (2002) The PYRIN-CARD protein ASC is an activating adaptor for Caspase-1. J. Biol. Chem., 277:21119-21122.

Stehlik, C., Lee, S.H., Dorfleutner, A., Stassinopoulos, A., Sagara, J., and Reed, J.C. (2003) Apoptosis-associated speck-like protein containing a caspase recruitment domain is a regulator of procaspase-1 activation. J. Immunol., 171:6154-6163.

Stehlik, C. (2007) The PYRIN domain in signal transduction. Curr Protein Pept Sci. 
Takeuchi O, K.A., Grote K, Kawai T, Hoshino K, Morr M, Mühlradt PF, Akira S. (2000) Cutting edge: preferentially the R-stereoisomer of the mycoplasmal lipopeptide macrophage-activating lipopeptide-2 activates immune cells through a toll-like receptor 2- and MyD88-dependent signaling pathway. $\mathrm{J}$ Immunol, 164:554-557.

Taniguchi S, S.J. (2007) Regulatory molecules involved in inflammasome formation with special reference to a key mediator protein, ASC. seminars in immunopathology, 29:231-238.

Thornberry, N.A., Bull, H.G., Calaycay, J.R., Chapman, K.T., Howard, A.D., Kostura, M.J., Miller, D.K., Molineaux, S.M., Weidner, J.R., Aunins, J., Elliston, K.O., Ayala, J.M., Casanoparallel, F.J., Chin, J., Ding, G.J.F., et al. (1992) A novel heterodimeric cysteine protease is required for interleukin-1 beta processing in monocytes. Nature, 356:768-774.

Tschopp, J., Martinon, F., and Burns, K. (2003) NALPs: a novel protein family involved in inflammation. Nat. Rev. Mol. Cell. Biol., 4:95-104.

Vinzing M, E.J., Lippmann J, Hocke AC, Zahlten J, Slevogt H, N'guessan PD, Günther S, Schmeck B, Hippenstiel S, Flieger A, Suttorp N, Opitz B. (2008) NAIP and Ipaf control Legionella pneumophila replication in human cells. J Immunol, 180:6808-6815.

Waites KB, T.D. (2004) Mycoplasma pneumoniae and its role as a human pathogen. Clin Microbiol Rev, 17:697-728.

Yamamoto, M., Yaginuma, K., Tsutsui, H., Sagara, J., Guan, X., Seki, E., Yasuda, K., Yamamoto, M., Akira, S., Nakanishi, K., Noda, T., and Taniguchi, S. (2004) ASC is essential for LPS-induced activation of procaspase-1 independently of TLR-associated signal adaptor molecules. Genes Cells, 9:1055-1067.

Yu HB, F.B. (2008) The caspase-1 inflammasome: a pilot of innate immune responses. Cell Host and Microbe, 4:198-208.

Yu, J.W., Wu, J., Zhang, Z., Datta, P., Ibrahimi, I., Taniguchi, S., Sagara, J., Fernandes-Alnemri, T., and Alnemri, E.S. (2006) Cryopyrin and pyrin activate caspase-1, but not NF-kappaB, via ASC oligomerization. Cell Death Differ, 13:236-249. 


\section{Study 3:}

Identification and Characterization of Three Novel ASC Isoforms 


\section{ABSTRACT}

Generation of mature IL-1 beta requires the activation of caspase-1, which occurs in multi-protein complexes termed inflammasomes. Apoptosis associated speck-like protein containing a caspase-recruitment domain (ASC), functions as a crucial adaptor protein in inflammasome assembly, and gene ablation studies have consistently demonstrated it is necessary for caspase-1 activation in response to a large number of pathogenic stimuli as well as endogenous danger signals. Structurally, ASC consists of two protein interaction domains; an Nterminal PYD, which is necessary for interaction with upstream cytosolic NLR receptors, and a C-terminal CARD domain, which is required for it interaction with caspase-1. Here, we identify three novel ASC isoforms. ASCb retains both an in tact PYD and CARD domains, but is lacking the linker region, which joins them. In contrast, ASCc possesses a large in-frame deletion in the PYD, and ASCd consists of only the first thirty-five amino acid residues of the PYD, fused to a novel peptide sequence. We characterize these isoforms with regards to their subcellular distribution, their ability to interact with NLRP3 and caspase-1, and evaluate their capacity to function as an inflammasome adaptor protein. 


\section{INTRODUCTION}

Apoptosis associated speck-like protein containing a caspase recruitment domain (ASC) is a small adaptor protein, which functions in both apoptotic (McConnell, 2000,McConnell, 2004,Ohtsuka, 2004,Parsons MJ, 2006) and inflammatory pathways. Structurally, ASC consists of an N-terminal PYRIN domain and a C-terminal caspase recruitment domain (CARD), which are both members of the death domain fold super family and function as protein interaction motifs. Both of these domains are required for ASC oligomerization and the formation of the characteristic perinuclear aggregates, which occur upon ectopic expression of ASC (Masumoto, 2001).

In innate immunity, ASC functions as an essential adaptor protein in the activation of caspase- 1 , which is essential for the maturation of the proinflammatory cytokines IL-1 beta and IL-18 (Mariathasan, 2004,Srinivasula, 2002,Stehlik, 2003b,Yamamoto, 2004). In addition, it can also function as a modulator of NF-kB activity (Sarkar, 2006,Stehlik, 2002). Caspase-1 is activated upon the assembly of multi-protein complexes termed inflammasomes, which form in response to pathogenic as well as endogenous danger signals (Martinon, 2002). Caspase-1 activation results in the subsequent processing of the proinflammatory cytokines IL-1beta and IL-18 from their inactive precursor forms to their active, secreted forms (Martinon F, 2007, Yu HB, 2008). Through protein interactions mediated by both its PYD and CARD domains, ASC serves as an essential link between pathogen recognition and caspase-1 activation (Taniguchi 
S, 2007). Inflammasome assembly and activation is initiated when cytosolic Nod-like receptors (NLRs), which function as pattern recognition receptors, are activated in response to specific pathogen associated molecular patterns (PAMPs) such as bacterial RNA (Kanneganti, 2006), or endogenous danger associated molecular patterns, such as extracellular ATP (Mariathasan, 2006). Activation of these receptors results in an ATP-dependent oligomerization and the recruitment of ASC via homotypic PYD-PYD interaction (Duncan JA, 2007,Faustin B, 2007). Subsequently, ASC uses its CARD to recruit caspase-1 molecules to the complex, which can then be activated by an induced proximity mechanism.

Post-transcriptional modifications, such as alternative splicing are a common occurrence in eukaryotic cells, and as much as $94 \%$ of all human genes undergo alternative splicing (Wang ET, 2008). They allow for enhanced protein diversity without requiring additional genes. Alternative RNA splicing is a particularly common occurrence in genes, which regulate apoptotic and inflammatory pathways (Leeman JR, 2008,Schwerk C, 2005). It enables cells to generate multiple proteins from a single mRNA transcript. For example, shorter isoforms of the proteins Rip2 and Nod2 have been identified (Krieg A, 2009 ,Rosenstiel P, 2006). Both of these proteins have been found to participate in the activation of the transcription factor NF-kB as well as caspase-1. Furthermore, in the case of Nod2, the shorter isoform was found to function as an antagonist for both Nod2-mediated NF-kB actiation and IL-1beta maturation. 
In this study, we report the identification and characterization of three novel structural isoforms of ASC, which we designated ASCb, ASCc, and ASCd. The structural modifications of the three additional isoforms are varied and include a deletion of the central linker domain, an in-frame deletion of a large portion of the PYD, and a truncated PYD followed by the replacement of the remainder of the protein with a new peptide. These structural alterations resulted in very different subcellular localization patterns, which were distinct from each other as well as from full-length ASCa. In addition, the ability to interact with the known binding partners NALP3 and pro-caspase- 1 was impacted as a result of structural changes observed with ASCc and ASCd, but not ASCb. Furthermore, while ASCb still retains still retains some ability to serve as an adaptor in the caspase-1-activating inflammasome, ASCc and ASCd are completely unable to function in this capacity, but may be involved in "fine-tuning" inflammasome responses. 


\section{EXPERIMENTAL PROCEDURES}

\section{Cell Culture.}

HEK 293 cells were obtained from the American Type Culture Collection and maintained in a humidified incubator at $37^{\circ} \mathrm{C}$ with $5 \% \mathrm{CO}_{2}$. They were propagated in DMEM supplemented with 10\% FBS, $2 \mathrm{mM}$ L-glutamine, $0.1 \mathrm{mM}$ non-essential amino acids, $1 \mathrm{mM}$ sodium pyruvate, $1.5 \mathrm{~g} / \mathrm{L}$ sodium bicarbonate, and $1 \%$ penicillin/streptomycin antibiotics. HEK 293 T cells were transiently transfected using Polyfect (Qiagen) or Superfect (invitrogen) according the procedures recommended by the manufacturer.

\section{Expression Plasmids}

A search of the publicly available expressed sequence tag (EST) database revealed three potential ASC isoforms ASCb (accession number BM456838), ASCc (accession number BE560228), and ASCd (accession number BM920038). The complete open reading frame of each isoform was amplified by PCR. ASC isoforms $b$ and $d$ were amplified using the primers [5'-CGGAATTC GATCCTGGAGCCATGGG-3'(forward): 5'-CGCTCGAGTGACCGGAGTGT TGCTG-3' (reverse) for 40 cycles. ASCc was amplified using the primers [5'CGGAATTCGATCCTG GAGCCATGGG-3'(forward): 5'-CGCTCGAGCCGGTG CTGGTCTATAA-3' (reverse). They were subsequently cloned into a pcDNA3 vector (Invitrogen) possessing an $\mathrm{N}$-terminal myc -epitope tag 
All other expression constructs (ASC, pro-IL-1beta, pro-caspase-1, NALP3R260W) have been previously described (Chu, 2001,Damiano, 2001,Lee, 2001,Stehlik, 2003b).

\section{Measurement of secreted IL-1beta from a reconstituted inflammasome} 293T cells were counted and seeded into type-I collagen-coated 12-well dishes, and allowed to adhere overnight. They were co-transfected in triplicate the following day with expression constructs encoding the constitutively active NALP3 ${ }^{\text {R260W }}$ mutant $(0.675 \mathrm{ug})$, pro-caspase-1 $(0.15 \mathrm{ug})$, and mouse pro-IL-1 beta (0.375ug), and each of the identified ASC isoforms (0.04ug ASCa and ASCb, and 0.15ug ASCc and ASCd), either alone or in the presence of full-length ASC. The total amount of DNA was kept constant with the addition of an empty pcDNA3 vector as necessary. The media was replaced 24 hours posttransfection, and at 48 hours post transfection, the supernatants were collected, centrifuged at $13,000 \mathrm{rpm}$ for $15 \mathrm{~min}$ at $4^{\circ} \mathrm{C}$, in order to remove any detached cells. The supernatants were subsequently stored at $-80^{\circ} \mathrm{C}$ or immediately analyzed by ELISA for IL-1 beta release according to the manufacturer's protocol (BD Biosciences). 


\section{Immunofluorescence}

293T cells were seeded onto Type I collagen-coated glass coverslips in 6-well plates, and allowed to adhere overnight. The following day they were transfected with plasmids encoding each of the ASC isoforms alone or in addition to GFPNALP3R260W, GFP-pro-caspase-1 (C/A mutant), or full-length HA-tagged ASC. Forty-eight hours post-transfection, they were fixed in 3.7\% paraformaldehyde for 30 minutes at room temperature. Following two washes in PBS and a five minute incubation in $50 \mathrm{mM}$ glycine, the cells were blocked and permeabilized in $0.5 \%$ saponin/ $1.5 \%$ normal goat serum/ $1 \%$ BSA/ PBS for thirty minutes. They were then immunostained using a polyclonal anti-myc antibody (Santa Cruz 1:200) or a monoclonal anti-myc antibody (Millipore 1:200). A polyclonal anti-HA antibody (Santa Cruz) was also used. After washing in $0.5 \%$ saponin/PBS, cells were incubated in blocking solution containing an AlexaFluor 488 or 546 conjugated secondary antibody directed against either mouse or rabbit (1:200). ToPro-3 (1:200) and AlexaFluor 546 conjugated phalloidin (1:100) were used to visualize the nucleus (DNA), and the actin cytoskeleton respectively. Images were obtained using laser scanning confocal microscopy (Zeiss LSM 510) under a $100 x$ oil-immersion objective.

\section{In vitro protein-interaction assay}

All ASC isoforms were in vitro translated and biotinylated using a TNT Quick Coupled Transcription/Translation kit (Promega) according to the manufacturer's 
protocol. GST-caspase-1-CARD was purified from E. coli DH5-alpha cells, following induction with $1 \mathrm{mM}$ IPTG for 4 hours at room temperature. Cells were resuspended in $2 \mathrm{~mL}$ STS buffer (10mM Tris pH 8.0, 1mM EDTA, and 150mM $\mathrm{NaCl}$ ) for every $100 \mathrm{~mL}$ of culture, and lysed by several rapid freeze/thaw cycles followed by the addition of $1 \mathrm{mg} / \mathrm{mL}$ of lysozyme. After a 30 minute incubation on ice, $10 \mathrm{mM}$ of DTT and $1.4 \%$ sodium sarkosyl were added, and the lysate was carefully sonicated, and cleared by centrifugation at $13,000 \mathrm{rpm}$ for 15 minutes. The extraction procedure was repeated, supernatants were pooled, adjusted to 4\% Triton X-100 was added and incubated with immobilized glutathione sepharose overnight at $4^{\circ} \mathrm{C}$. Beads were washed three times with PBS and stored at $4^{\circ} \mathrm{C}$ in $\mathrm{PBS} / 0.1 \%$ sodium azide.

The immobilized GST proteins were blocked for 30 minutes at room temperature in HKMEN buffer $\left(142.4 \mathrm{mM} \mathrm{KCl}, 5 \mathrm{mM} \mathrm{MgCl}_{2}, 10 \mathrm{mM}\right.$ HEPES (pH 7.4), 0.5mM EGTA, 1mM EDTA, 0.2\% NP-40, 1mM DTT) supplemented with protease inhibitors (Roche) and $1 \mathrm{mg} / \mathrm{mL}$ BSA. Following one wash with HKMEN buffer, the beads were incubated overnight on a rotator with the in vitro translated ASC isoforms. Bound proteins were washed 4 times in HKMEN buffer supplemented with protease inhibitors, boiled in Laemmli buffer, separated by SDS/PAGE, transferred to a PVDF membrane and probed with a monoclonal anti-myc antibody (Millipore) followed by an HRP-conjugated anti-mouse secondary antibody. The blot was then developed with chemiluminescent reagents (Millipore). 


\section{RESULTS}

\section{Identification of three novel ASC isoforms}

A search of the publicly available EST database revealed the existence of three novel isoforms of ASC expressed in human tissues. The first isoform, $\mathrm{ASCb}$, is the most frequently expressed isoform with the exception of full-length ASC. This isoform is distinguished from full-length ASC by an in-frame deletion resulting in the loss of the linker domain, which corresponds to amino acid residues 93-111 (Fig. 1). Therefore, the resulting protein retains fully intact PYD and CARD domains. However, these domains are now directly fused together, rather than being separated by a linker region. The second isoform we identified, ASCc, was also generated by an in-frame deletion. In contrast to the deletion giving rise to $\mathrm{ASCb}$ that leaves both protein-interaction domains intact, the deletion in ASCc results in the loss of amino acid residues 26-85, which compose a large portion of the PYD (Fig. 1). However, both the linker domain and the CARD domain of this isoform remain unaffected. ASCd, retained the least amount of the full-length ASC structure. Interestingly, ASCd is composed of only the first thirty-five amino acid residues of ASC, which contain the first two alphahelices of the PYD. The remainder of the ASC protein has been completely deleted and replaced by sixty-nine novel amino acids (Fig. 1).

Furthermore, two of the three isoforms, $\mathrm{ASCb}$ and $\mathrm{ASC}$, were likely generated through the post-transcriptional process of alternative mRNA splicing as both of the regions deleted in these isoforms are flanked by potential splice 
donor (SD) and splice acceptor (SA) sites. These regions closely resemble the consensus sequences previously characterized for SD ([C/A]AGGT[A/G]AGT) and $\mathrm{SA}\left(\left[\mathrm{C} / \mathrm{T}_{10-11}\right] \mathrm{N}[\mathrm{C} / \mathrm{T}] \mathrm{AG}[\mathrm{G} / \mathrm{A}]\right.$ sites.

\section{ASC isoforms display different localization patterns from full-length ASC and each other.}

Previous studies with full-length ASC have revealed a very unique characteristic of this protein. In addition to diffuse nuclear and cytoplasmic subcellular distribution patterns, ectopic expression of full-length ASC results in the formation of perinuclear aggregates, which have been termed 'specks'. Furthermore, our laboratory recently demonstrated that while endogenous ASC is predominantly nuclear in resting macrophages, perinuclear aggregates of ASC are observed upon activation with inflammatory stimuli. Therefore, we first evaluated how the structural alterations observed with each of the ASC isoforms impacted the subcellular localization of these proteins.

Expression plasmids encoding each of the ASC isoforms were transiently transfected into HEK 293 cells, and their subcellular distribution was analyzed by immunofluorescent microscopy. As shown in Figure 2 (top panel), expression of full-length ASCa resulted in the formation of the previously characterized perinuclear aggregate. However, none of the other isoforms retained the capacity to form these structures. Rather, each isoform exhibited its own, unique localization pattern. ASCb displayed a diffuse, exclusively cytoplasmic 
subcellular distribution (Figure 2, second panel). This staining pattern further distinguishes it from ASCa, which is frequently found in the nucleus. ASCc was also found in the cytoplasm. However, this isoform oligomerized into long, filamentous structures rather than exhibiting a diffuse staining pattern (Fig. 2, third panel). Unlike ASCb and ASCc, ASCd was occasionally found in the nucleus. Furthermore, it displayed a more vesicular-type localization pattern, which was in contrast with the diffuse localization of $\mathrm{ASCb}$ and the oligomerized filaments of ASCc (Figure 2, bottom panel).

\section{ASC isoforms exhibit differences in their ability to interact with other inflammasome proteins, and function in IL-1beta processing.}

It has become well established that in its capacity as an inflammasome adaptor protein, ASC directly interacts with other components of this multi-protein complex. Additionally, previous studies performed by us as well as others have demonstrated that other inflammasome proteins, including upstream NLR receptors and pro-caspase-1, are recruited to perinuclear aggregates when coexpressed with ASCa. Furthermore, our examination of endogenous ASC revealed that both endogenous NLRP3 and caspase-1 are recruited into aggregates following activation with E. coli RNA. Therefore, we next examined whether each of the individual ASC isoforms could interact with other inflammasome proteins. HEK 293 cells were co-transfected with the constitutively active GFP-tagged NLRP3 ${ }^{\text {R260W }}$ mutant, and each isoform and 
subsequently analyzed for co-localization by immunofluorescence (Fig. 3A). As expected, full-length ASCa and NLRP3 ${ }^{\mathrm{R} 260 \mathrm{~W}}$ co-localized in the previously described perinuclear aggregates. In addition, $\mathrm{ASCb}$, which still retains a fully intact PYD, also co-localized with NLRP3 ${ }^{R 260 w}$. Intriguingly, co-expression of $A S C b$ with the upstream NLRP3 ${ }^{R 260 W}$ receptor altered its diffuse cytoplasmic localization pattern and resulted in the formation of cytoplasmic aggregates. However, while these aggregates did exhibit a perinuclear localization, they were not as small and condensed as those observed with ASCa. Finally, neither ASCc nor ASCd displayed any co-localization with upstream NLRP3 ${ }^{\mathrm{R} 260 \mathrm{~W}}$, as expected due to the deletion of the part of the PYD, which is necessary for this interaction.

Next, we evaluated whether each of the ASC isoforms were capable of interacting with pro-caspase-1 (C/A). We transiently co-transfected HEK 293 cells with GFP-pro-caspase-1 and each of the ASC isoforms. Since previous studies have demonstrated that cells may release active caspase-1, we utilized an enzymatically inactive C/A mutant. Following transfection, the cells were fixed, and co-localization was analyzed by immunofluorescence. ASCa, ASCb, and ASCc all co-localized with pro-caspase-1, while ASCd (Fig. 3B, bottom panel) did not, as expected due to its lack of the CARD. As observed with NLRP3, ASCa recruited caspase-1 into perinuclear aggregates (Fig. 3B, top panel). Additionally, caspase-1 was also found to co-localize with ASCc in the long filamentous structures formed by this isoform (Fig. 3B, third panel). ASCb, 
on the other hand, retained the diffuse, cytosolic localization pattern that it exhibited when expressed alone. Furthermore, it only co-localized with cytoplasmic caspase-1, as it was excluded from the nucleus (Fig. 3B, second panel). In addition, we also examined the ability of in vitro translated ASCa and ASCb to directly interact with the CARD domain of caspase-1. As shown in figure 3D, both ASCa and ASCb are capable of direct binding to the GST-tagged CARD domain of caspase-1.

Next, we wanted to determine how the different ASC isoforms impact their ability to function as an inflammasome adaptor protein. Therefore, we reconstituted the inflammasome in HEK 293 cells, which lack endogenous expression of all inflammasome components, but do possess the ability to release mature IL-1beta, once it is generated. Cells were transiently cotransfected with expression plasmids encoding pro-IL-1 beta, pro-caspase-1, and each of the ASC isoforms in the presence or absence of the constitutively active NLRP3 ${ }^{\text {R260W }}$. Culture supernatants were collected thirty-six hours posttransfection and analyzed for released IL-1beta by ELISA. As shown in figure 3, only those ASC isoforms, which possessed intact PYD and CARD domains, ASCa and $\mathrm{ASCb}$, were capable of forming a functioning inflammasome, and generating mature IL-1beta (Fig. 3C). Neither ASCc, nor ASCd were able to generate mature IL-1beta, which suggests that the structural alterations observed in these isoforms render them unable to function as an inflammasome adaptor protein. Intriguingly, loss of the linker region resulted in a decrease in the IL- 
1 beta secretion, indicating that while this isoform can function as an inflammasome adaptor, it is less efficient than full-length ASCa.

\section{Full-length ASC a co-localizes with ASCb and ASCc}

Previous studies have demonstrated that ASC is capable of selfinteraction via binding at either protein interaction domain. Furthermore, one of the major mechanisms of inflammasome regulation involves the disruption of the protein-protein interactions between NLRPs and ASC, or between ASC and caspase- 1 by pyrin only proteins (POPs) or CARD-only proteins (COPs) respectively. Therefore, we wanted to explore the possibility that ASC b and ASCc could interact with full-length ASCa, and potentially exert influence over inflammasome assembly and function. First, we utilized immunofluorescence to evaluate how co-expression of full-length ASCa, with either ASCb or ASCc impacted the subcellular localization of the isoforms. Interestingly, co-expression of ASC isoforms $a$ and $b$ resulted in the co-localization of these two proteins in perinuclear aggregates. However, the aggregates differed from those, which are observed upon overexpression of ASCa. Co-expression of ASCa and ASCb resulted in the formation of large, irregularly shaped perinuclear aggregates, rather than the small, circular structures formed by ASCa alone (Fig. 4, top panel). Co-expression of ASCc with full-length ASCa also altered its subcellular distribution. Instead of forming long filamentous structures, ASCc was recruited to the perinuclear ASCa aggregates. However, unlike those observed upon co- 
expression with $\mathrm{ASCb}$, these aggregates maintained all of the previously identified characteristics of an ASC 'speck' (Fig. 4, bottom panel). Intriguingly, this is the same localization pattern observed when ASCa is co-expressed with either a POP or a COP, suggesting that ASCc may compete with caspase- 1 for binding to the CARD domain of full-length ASCa. 


\section{DISCUSSION}

In this study, we report the identification of three novel isoforms of the inflammasome adaptor protein ASC, designated ASCb, ASCc, and ASCd. Two of these isoforms are most likely generated through alternative splicing of ASC mRNA, while the mechanism, which gives rise to ASCd remains unclear. Each isoform exhibits unique structural modifications, which have a significant impact on their localization as well as their ability to participate in inflammasome assembly. As a result, alternative mRNA splicing of ASC may represent a novel regulatory mechanism for inflammasome assembly, and subsequent IL-1beta release.

ASCb was the most commonly expressed novel isoform yielded by our search of the EST database. It was second only to full-length ASCa in its frequency of expression, suggesting that it is widely expressed in human tissues. While much work has been devoted to elucidating the structure and function of the two protein-interacting domains of ASC (Masumoto, 2001,Moriya, 2005,Stehlik, 2002,Stehlik, 2003b), no studies have been undertaken, which evaluate the role of the linker domain in the functional or localization properties of ASC. Discovery of a novel ASC isoform, which lacks this region, enabled us to evaluate for the first time the contribution of this domain to the characteristics of ASC.

Previous studies with ASC have shown that enforced overexpression of this protein results in the formation of perinuclear aggregates termed specks 
(Fig.2) (Masumoto, 1999,T Fernandes-Alnemri, 2007). In this study, we found that deletion of the linker domain prevented the formation of these structures. Rather, ASCb displayed a diffuse, cytosolic localization pattern. These results demonstrate that the linker domain is necessary for the spontaneous formation of perinuclear aggregates upon individual overexpression of ASC. However, coexpression with constitutively active NLRP3 ${ }^{\text {R260W }}$ (Fig. 3) or full-length ASCa, but not caspase-1 (Fig. 4), was able to restore aggregate formation, although these aggregates were larger and more irregular in shape than those observed with full-length ASCa. This data suggests that engagement of the PYD of ASCb with upstream NLRPs or ASCa can induce the aggregation of $\mathrm{ASCb}$. These results are consistent with previous studies, which have suggested that the PYD of ASC functions as the oligomerization domain, while the ASC CARD is the effector domain (Srinivasula, 2002).

Functionally, ASCb was the only novel ASC isoform, which exhibited the ability to serve as an adaptor protein in a reconstituted inflammasome, as measured by IL-1beta release. However, lack of a linker domain did render ASCb less efficient in this capacity, than the full-length ASCa (Fig. 3c). One possible explanation for these results is that the linker domain of ASC confers flexibility to the protein, which is necessary in order to efficiently aggregate with NLRP3 and caspase-1. This hypothesis is supported by our immunofluorescence data, which showed that co-expression of ASCa and ASCb not only resulted in aggregation of the previously diffuse $\mathrm{ASCb}$, but also resulted 
in disruption in the architecture of the full-length ASC aggregate. There is also less efficient recruitment of caspase- 1 into ASC-containing aggregates, and thus dampened inflammasome activity and IL-1beta maturation.

ASCc exhibited functional and localization properties, which differed from both ASCa and ASCb. Overexpression of ASCc resulted in the formation of long cytoplasmic filaments. The formation of these structures was not unexpected, as the ASCc protein possesses a severely truncated PYD, while leaving the linker and CARD domains intact. Thus, this isoform is essentially comprised of an individual CARD domain, a member of the death domain fold superfamily, and previous studies have revealed that long filamentous structures are the result of ectopic expression of individual members of this family (Masumoto, 2001,Siegel, 1998). Also as predicted, this isoform retained the ability to co-localize with caspase-1, which was also recruited into the filaments, while co-localization with $\mathrm{NALP}^{\mathrm{R} 260 \mathrm{~W}}$ was not observed. These results are consistent with previous work demonstrating that the interaction between ASC and caspase- 1 is mediated by the CARD (Srinivasula, 2002,Stehlik, 2003b), while the interaction between ASC is upstream NLRs is mediated by the PYD (Stehlik, 2007). In addition to colocalization with caspase-1, ASCc was also able to co-localize with full-length ASCa. Furthermore, ASCc was recruited into the perinuclear aggregates, although without disrupting their structure, as occurred with co-expression of ASCb. 
Functionally, ASCc was completely deficient in the capacity as an inflammasome adaptor protein. In a reconstituted inflammasome, it was incapable of eliciting IL-1beta release above basal levels. Structurally, this protein most closely resembles that of a CARD-only-protein (COP). This group of proteins includes several proteins including COP, ICEBERG, Pseudo-ICE, and INCA, and is characterized by the fact that all members are composed solely of a CARD domain (Druilhe, 2001,Humke, 2000,Lamkanfi, 2004,Lee, 2001,Stehlik C, 2007). Like their PYD-only couterparts (POPs), these proteins function as dominant-negative antagonists of the inflammasome. However, unlike POPs, COPs prevent inflammasome activation via the disruption of ASC-caspase-1 interaction, rather than blocking the interaction between NLRs and ASC (Bedoya F, 2007,Dorfleutner, 2007a,Dorfleutner, 2007b,Stehlik, 2003a). The similarities between ASCc and COPs raised the intriguing possibility that ASCc may also function as an inflammasome inhibitor. This hypothesis is supported by our data showing that ASCc co-localizes with both caspase-1 and full-length ASCc, suggesting an interaction with one or both of these proteins. Furthermore, ASCc was recruited into the perinuclear aggregates formed by ASCa.

ASC $d$ is the most unusual of the novel ASC isoforms we identified. It is the smallest of the isoforms we identified and its retention of only the first thirtyfive amino acid residues of the PYD, comprising the alpha helices 1 and 2 makes it the isoform. Like ASCb and ASCc, ASCd was predominantly cytoplasmic (Fig. 2, bottom panel). However, we did occasionally observe this isoform in the 
nucleus (data not shown). Furthermore, while it did not form an ASC 'speck', this protein was frequently found to coalesce into multiple punctate structures (Figs. 2 and 3). As predicted by its lack of a CARD domain, ASCd did not co-localize with caspase-1 (Fig. 3b). Additionally, it also did not co-localize with NLRP3 ${ }^{\text {R260W }}$, suggesting that the portion of the PYD expressed by this isoform was insufficient to mediate an interaction with this NLR (Fig. 3a), which usually requires residues in alpha-helices 1 and 4 , or 2 and 3 . This result is consistent with recent evidence published by Srimathi et al. predicting that the interaction between ASC and NLRP3 occurs at a positive electrostatic potential surface patch (EPSP) on the PYD of ASC formed by Lys ${ }^{21}, \mathrm{Lys}^{22}, \mathrm{Lys}^{26}$ and $\mathrm{Arg}^{41}$, and a negative EPSP on NLRP3 (Srimathi T, 2008). While ASCd does possess the three key lysine residues, it no longer contains an arginine at position 41. Therefore, the structural alterations resulting in the loss of this key amino acid residue likely caused a disruption of the positive EPSP and rendered this protein incapable of interacting with NLRP3. The inability of this protein to interact with the key inflammasome components caspase-1 and NLRP3 is further supported by the lack of IL-1beta released upon reconstitution of the inflammasome with ASCd, rather than full-length ASCa (Fig. 3c).

Overall, our data suggest that alternative splicing of the inflammasome adaptor ASC resulting in multiple isoforms of this protein may serve as a novel regulatory mechanism for "fine-tuning" inflammasome responses. We have identified and characterized three novel isoforms with 
regards to their subcellular distribution as well as their ability to function in inflammasome assembly. Furthermore, ASCb provided us with a tool to evaluate the role of the linker domain in inflammasome assembly, and our data show that it is required for maximal activation of the inflammasome. Additionally, ASCc may represent a novel COP, which functions as an inhibitor of inflammasome formation through the disruption of the ASCa and caspase-1 interaction. Future studies are needed to better characterize the significance of ASC splicing in addition to determining the specific cell types and tissues, which express these proteins, and their possible influence on other functions of ASC, such as its role in apoptosis. 
A
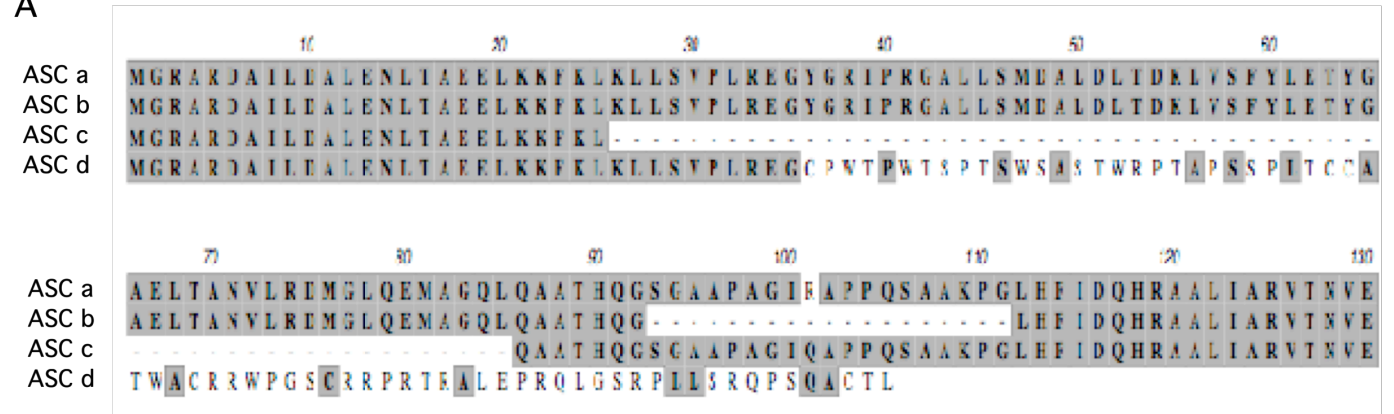

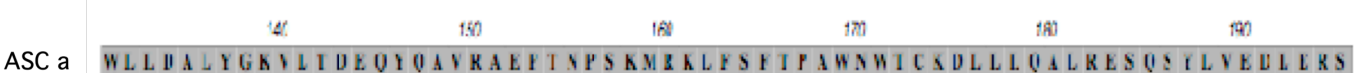

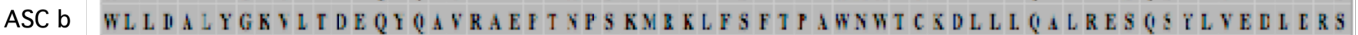

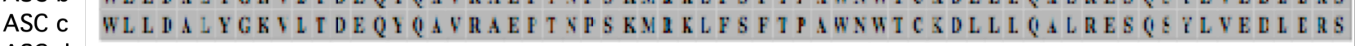

B

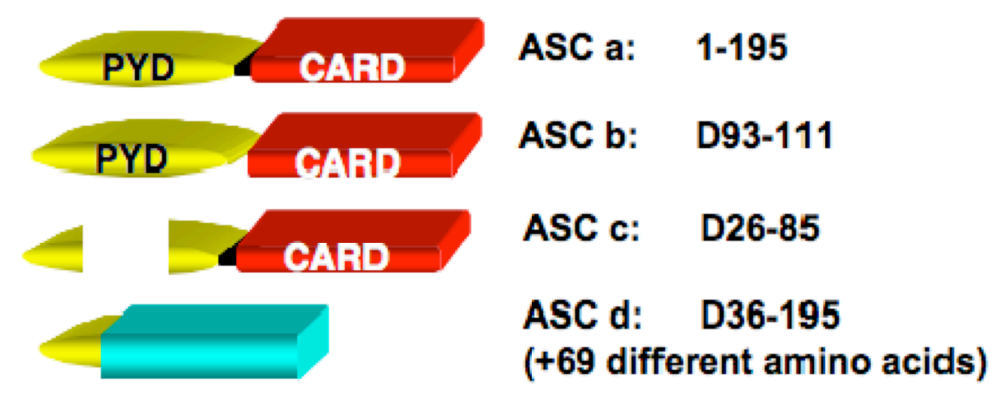

Figure 1. Structural alterations in the protein organization of ASC isoforms (A) Clustal alignment of four different ASC isoforms. ASC a represents the previously characterized full-length ASC protein consisting of an $\mathrm{N}$-terminal PYD, a central linker domain, and a C-terminal CARD domain. ASC b represents a splice variant in which the central linker domain corresponding to amino acid residues 93-111 has been deleted. ASC c represents a splice variant in which a large portion of the N-terminal PYD corresponding to amino acid residues 26-85, has been removed. ASC d represents an isoform, which possesses only the first 35 amino acid residues of the ASC PYD. The remainder of the protein has been replaced by a novel 69 amino acid peptide.

(B). Schematic representation of the ASC isoforms demonstrating the regions of the protein, which have been removed or altered. 


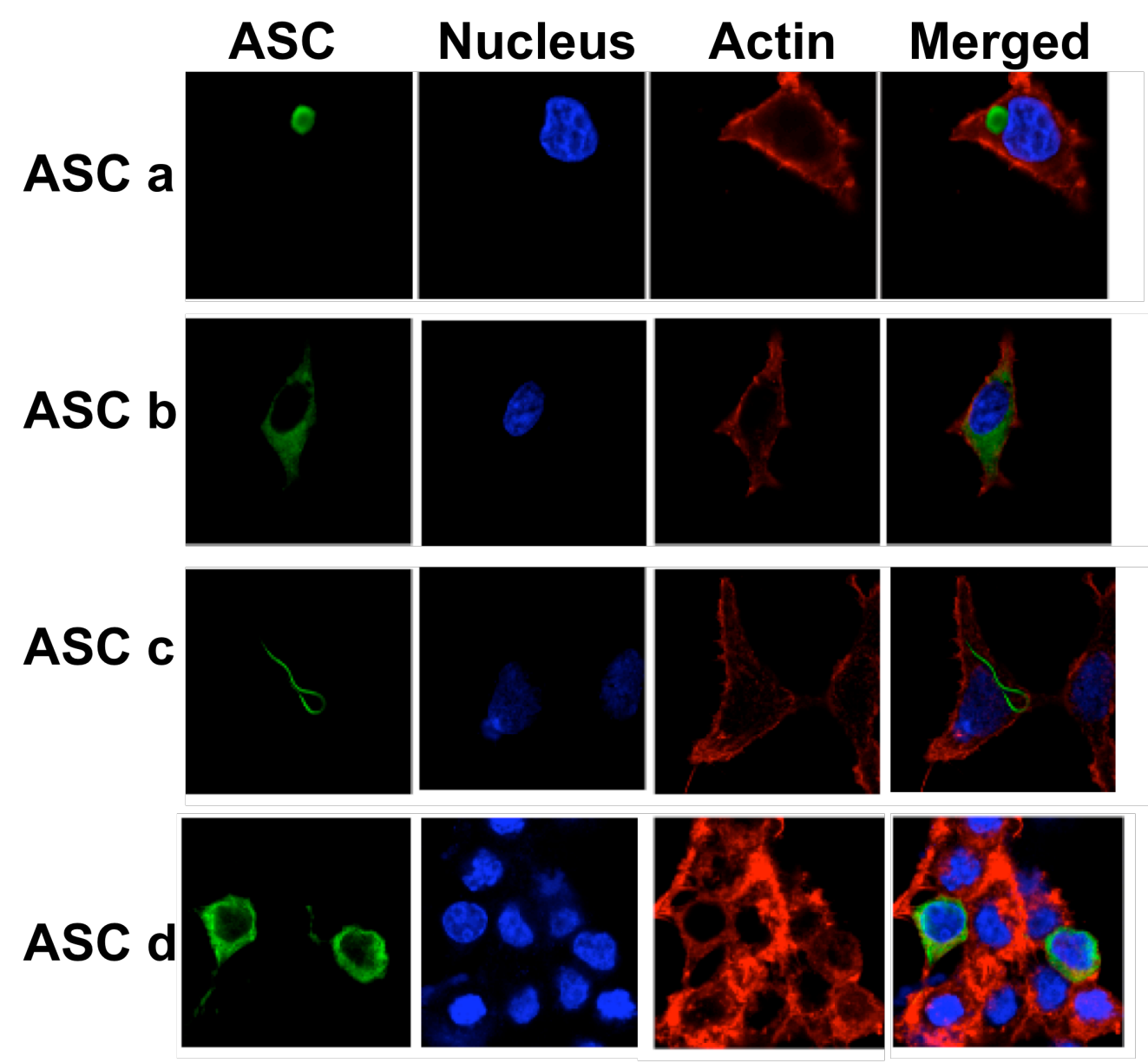

Figure 2. Subcellular distribution of ASC isoforms

Subcellular localization of each ASC isoform was examined in HEK 293T cells, which were transiently transfected with plasmids encoding individual ASC isoforms with an $\mathrm{N}$-terminal myc epitope tag. They were subsequently fixed and immunostained with a polyclonal anti-myc antibody (Santa Cruz) followed by an AlexaFluor-488 conjugatetd anti-rabbit secondary antibody. Topro-3 and AlexaFluor 546 conjugated phalloidin were used to visualize the nucleus and actin cytoskeleton respectively. Laser scanning confocal microscopy with a 100x objective was used to obtain all images. Panels from left to right demonstrate the localization of the ASC isoforms (green), nuclei (blue), the actin cytoskeleton (red), and a merged composite image. 

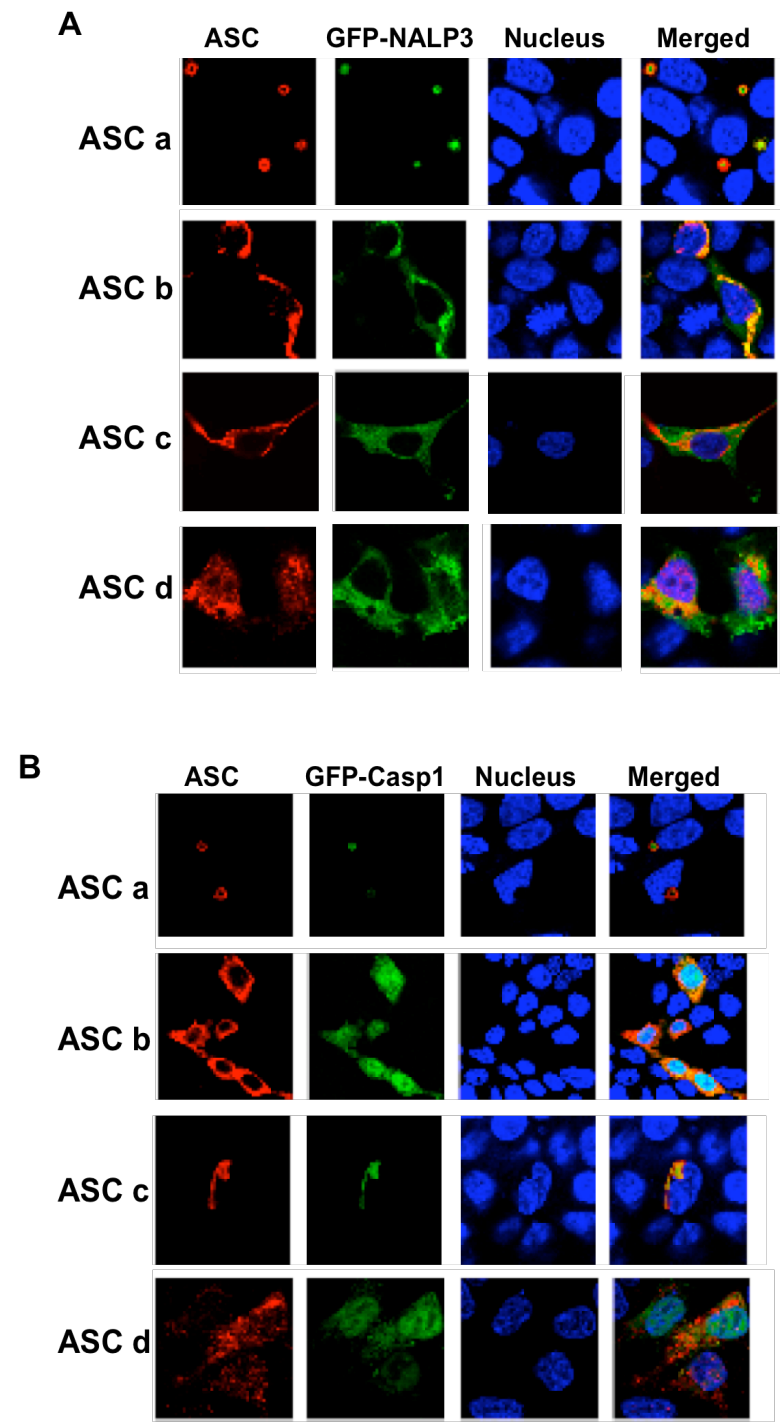

Figure 3. ASC isoforms exhibit different co-localization patterns with o ther key inflammasome proteins and have a diminished capacity to elicit inflammasome activation.

HEK 293 cells were transiently co-transfected using Polyfect (Qiagen) with individual ASC isoforms and either GFP-tagged NALP3 ${ }^{\text {R260W }}$ (A), or GFP-tagged pro-caspase-1 C/A mutant (B). They were subsequently fixed and immunostained with a polyclonal anti-myc antibody (Santa Cruz) and AlexaFluor 546-conjugated anti-rabbity secondary antibody. Topro-3 was used to visualize the nucleus. All images were acquired using laser scanning confocal microscopy with a 100x oil-immersion objective. Panels from left to right show ASC isoforms (red), GFP-NLRP3 ${ }^{(\mathrm{R} 260 \mathrm{~W})}(\mathrm{A})$ or GFP-pro-caspase-1(C/A) (B) (green), nuclei (blue), and a merged image. 
C

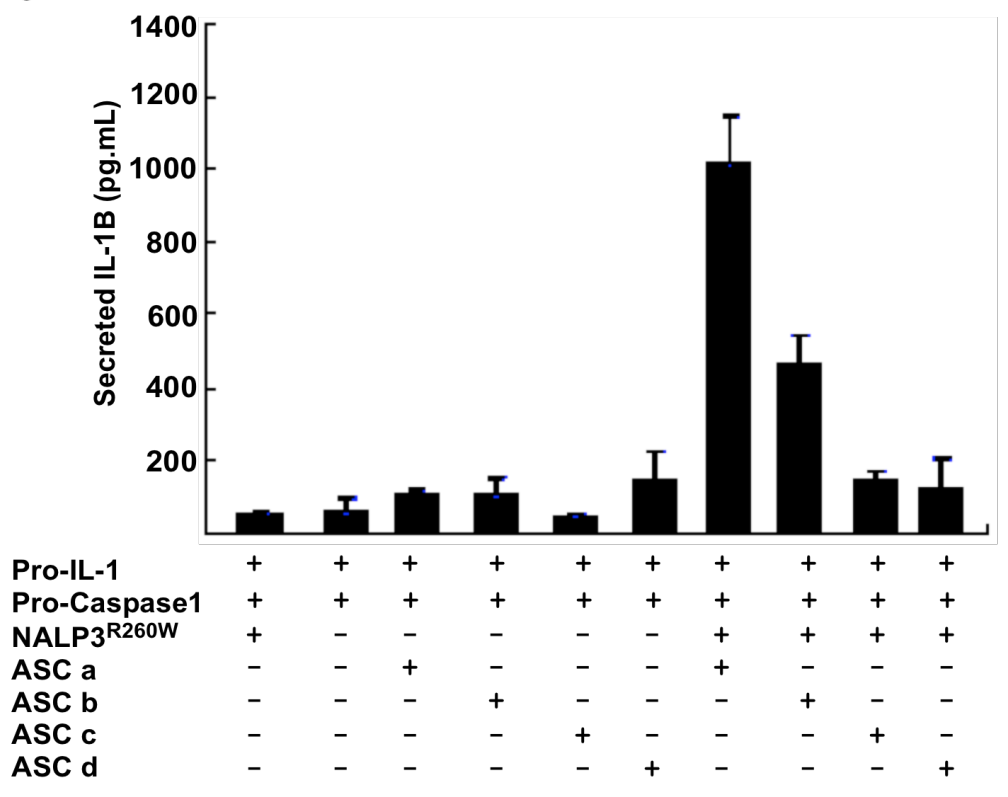

D

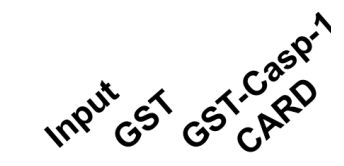

ASC a

ASC $b$

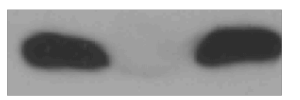

(C). Inflammasomes consisting of a constitutively active NALP3 ${ }^{\text {R260W }}$, pro-IL1 beta, pro-caspase-1, and one of the ASC isoforms were transiently reconstituted in HEK 293 cells as indicated using Superfect (Invitrogen). 48 hours post-transfection, the supernatants were collected, cleared by centrifugation, and analyzed for secreted IL-1beta by ELISA. Results represent an average of three independent experiments +/- SD and are presented as $\mathrm{pg} / \mathrm{mL}$ of secreted IL-1beta.

(D). In vitro binding between ASC a, ASC b, and caspase-1 CARD. Myc-tagged ASC $a$ and ASC b were in vitro translated, and subjected to GST pull-down assays using GST-Caspase-1 CARD, and a GST control. Protein complexes were separated by SDS-PAGE, transferred to a PVDF membrane, and visualized by immunoblotting with anti-myc antibody. 

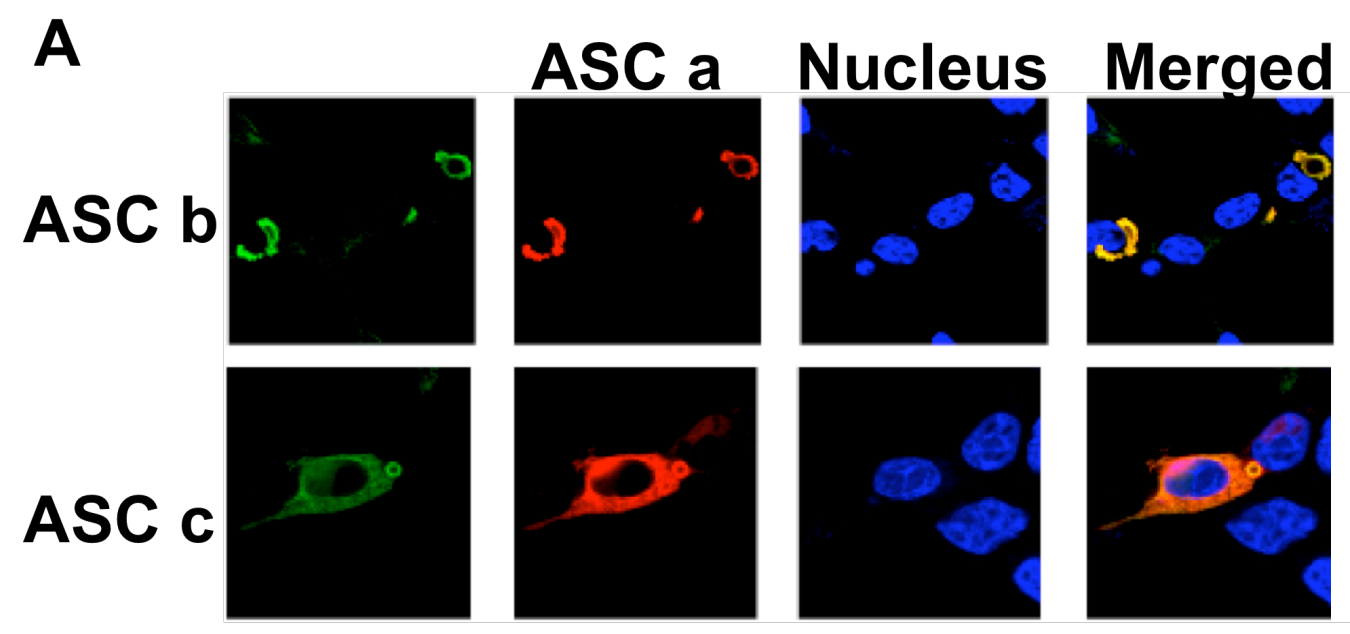

Figure 4. Co-localization of ASC $b$ and ASC $c$ with the full length ASC a. HEK 293 cells were transiently co-transfected using Polyfect (Qiagen) with individual ASC $b$ or ASC $c$ and an HA-tagged full-length ASC a. They were subsequently fixed and immunostained with a monoclonal anti-myc antibody (Millipore) and a polyclonal anti-HA antibody (Abcam) followed by AlexaFluor 488 anti-mouse and AlexaFluor 546-conjugated anti-rabbity secondary antibodies. Topro-3 was used to visualize the nucleus. All images were acquired using laser scanning confocal microscopy with a 100x oil-immersion objective. Panels from left to right show ASC b or ASC c (green), ASC a (red), nuclei (blue), and a merged image. 


\section{REFERENCES}

Bedoya F, S.L., Harton JA. (2007) Pyrin-only protein 2 modulates NF-kappaB and disrupts ASC:CLR interactions. Journal of Immunology, 178:38373845.

Chu, Z.-L., Pio, F., Xie, Z., Welsh, K., Krajewska, M., Krajewski, S., Godzik, A., and Reed, J.C. (2001) A novel enhancer of the Apaf1 apoptosome involved in cytochrome c-dependent caspase activation and apoptosis. J. Biol. Chem., 276:9239-9245.

Damiano, J.S., Stehlik, C., Pio, F., Godzik, A., and Reed, J.C. (2001) CLAN, a novel human CED-4-like gene. Genomics, 75:77-83.

Dorfleutner, A., Bryan, N.B., Talbott, S.J., Funya, K.N., Rellick, S.L., Reed, J.C., Shi, X., Rojanasakul, Y., Flynn, D.C., and Stehlik, C. (2007a) Cellular PYRIN domain-only protein (cPOP) 2 is a candidate regulator of inflammasome activation. Infection and Immunity, in press.

Dorfleutner, A., McDonald, S.J., Bryan, N.B., Funya, K.N., Reed, J.C., Shi, X., Flynn, D.C., Rojanasakul, Y., and Stehlik, C. (2007b) A Shope Fibroma virus PYRIN-only protein modulates the host immune response. Virus Genes, in press.

Druilhe, A., Srinivasula, S.M., Razmara, M., Ahmad, M., and Alnemri, E.S. (2001) Regulation of IL-1beta generation by Pseudo-ICE and ICEBERG, two dominant negative caspase recruitment domain proteins. Cell Death Differ., 8:649-657.

Duncan JA, B.D., Wang Y, Willingham SB, Ye Z, Zimmermann AG, Ting JP. (2007) Cryopyrin/NALP3 binds ATP/dATP, is an ATPase, and requires ATP binding to mediate inflammatory signaling. Proc Natl Acad Sci U S A, 104:8041-8046.

Faustin B, L.L., Bruey JM, Luciano F, Sergienko E, Bailly-Maitre B, Volkmann N, Hanein D, Rouiller I, Reed JC. (2007) Reconstituted NALP1 inflammasome reveals two-step mechanism of caspase-1 activation. Molecular Cell, 25:713-724.

Humke, E.W., Shriver, S.K., Starovasnik, M.A., Fairbrother, W.J., and Dixit, V.M. (2000) ICEBERG: a novel inhibitor of interleukin-1beta generation. Cell, 103:99-111. 
Kanneganti, T.D., Ozoren, N., Body-Malapel, M., Amer, A., Park, J.H., Franchi, L., Whitfield, J., Barchet, W., Colonna, M., Vandenabeele, P., Bertin, J., Coyle, A., Grant, E.P., Akira, S., and Nunez, G. (2006) Bacterial RNA and small antiviral compounds activate caspase-1 through cryopyrin/Nalp3. Nature, 440:232-236.

Krieg A, L.N.G., Reed JC. (2009) RIP2-beta: A novel alternative mRNA splice variant of the receptor interacting protein kinase RIP2. Mol Immunol, 46:1163-1170.

Lamkanfi, M., Denecker, G., Kalai, M., D'Hondt, K., Meeus, A., Declercq, W., Saelens, X., and Vandenabeele, P. (2004) INCA, a novel human caspase recruitment domain protein that inhibits interleukin-1 beta generation. J. Biol. Chem., 279:51729-51738.

Lee, S.-H., Stehlik, C., and Reed, J.C. (2001) COP, a CARD-containing protein and inhibitor of pro-interleukin-1b processing. J Biol Chem, 276:3449534500 .

Leeman JR, G.T. (2008) Alternative splicing in the NF-kappaB signaling pathway. Gene, 423:97-107.

Mariathasan, S., Newton, K., Monack, D.M., Vucic, D., French, D.M., Lee, W.P., Roose-Girma, M., Erickson, S., and Dixit, V.M. (2004) Differential activation of the inflammasome by caspase-1 adaptors ASC and Ipaf. Nature, 430:213-218.

Mariathasan, S., Weiss, D.S., Newton, K., McBride, J., O'Rourke, K., RooseGirma, M., Lee, W.P., Weinrauch, Y., Monack, D.M., and Dixit, V.M. (2006) Cryopyrin activates the inflammasome in response to toxins and ATP. Nature, 440:228-232.

Martinon, F., Burns, K., and Tschopp, J. (2002) The Inflammasome: A molecular platform triggering activation of inflammatory caspases and processing of prolL-b. Mol. Cell, 10:417-426.

Martinon F, T.J. (2007) Inflammatory caspases and inflammasomes: master switches of inflammation. Cell Death \& Differ, 14:10-22.

Masumoto, J., Taniguchi, S., Ayukawa, K., Sarvotham, H., Kishino, T., Niikawa, N., Hidaka, E., Katsuyama, T., Higuchi, T., and Sagara, J. (1999) ASC, a novel 22-kDa protein, aggregates during apoptosis of human promyelocytic leukemia HL-60 cells. J. Biol. Chem., 274:33835-33838. 
Masumoto, J., Taniguchi, S., and Sagara, J. (2001) Pyrin N-terminal homology domain- and caspase recruitment domain-dependent oligomerization of ASC. Biochem. Biophysical. Res. Commun., 280:652-655.

McConnell, B.B., and Vertino, P.M. (2000) Activation of a caspase-9-mediated apoptotic pathway by subcellular redistribution of the novel caspase recruitment domain protein TMS1. Cancer Res., 60:6243-6247.

McConnell, B.B., and Vertino, P.M. (2004) TMS1/ASC: The cancer connection. Apoptosis, 9:5-18.

Moriya, M., Taniguchi, S., Wu, P., Liepinsh, E., Otting, G., and Sagara, J. (2005) Role of charged and hydrophobic residues in the oligomerization of the PYRIN domain of ASC. Biochemistry, 44:575-583.

Ohtsuka, T., Ryu, H., Minamishima, Y.A., Macip, S., Sagara, J., Nakayama, K.I., Aaronson, S.A., and Lee, S.W. (2004) ASC is a Bax adaptor and regulates the p53-Bax mitochondrial apoptosis pathway. Nat. Cell. Biol., 6:121-128.

Parsons MJ, V.P. (2006) Dual role of TMS1/ASC in death receptor signaling. Oncogene, 25:6948-6958.

Rosenstiel P, H.K., Till A, Hampe J, Hellmig S, Sina C, Billmann S, von Kampen O, Waetzig GH, Platzer M, Seegert D, Schreiber S. (2006) A short isoform of NOD2/CARD15, NOD2-S, is an endogenous inhibitor of NOD2/receptor-interacting protein kinase 2-induced signaling pathways. Proc Natl Acad Sci U S A, 103:3280-3285.

Sarkar, A., Duncan, M., Hart, J., Hertlein, E., Guttridge, D.C., and Wewers, M.D. (2006) ASC Directs NF-\{kappa\}B Activation by Regulating Receptor Interacting Protein-2 (RIP2) Caspase-1 Interactions. J Immunol, 176:49794986.

Schwerk C, S.-O.K. (2005) Regulation of apoptosis by alternative pre-mRNA splicing. Mol Cell, 19:1-13.

Siegel, R., Martin, D., Zheng, L., Ng, S., Bertin, J., Cohen, J., and Lenardo, M. (1998) Death-effector filaments: novel cytoplasmic structures that recruit caspases and trigger apoptosis. J. Cell Biol., 141:1243-1253.

Srimathi T, R.S., Dubas RL, Chang H, Cheng H, Roder H, Park YC. (2008) Mapping of POP1-binding site on pyrin domain of ASC. Journal of Biological Chemistry, 283:15390-15398. 
Srinivasula, S.M., Poyet, J.-L., Razmara, M., Datta, P., Zhang, Z., and Alnemri, E.S. (2002) The PYRIN-CARD protein ASC is an activating adaptor for Caspase-1. J. Biol. Chem., 277:21119-21122.

Stehlik, C., Fiorentino, L., Dorfleutner, A., Bruey, J.M., Ariza, E.M., Sagara, J., and Reed, J.C. (2002) The PAAD/PYRIN-family protein ASC is a dual regulator of a conserved step in nuclear factor kappaB activation pathways. J. Exp. Med., 196:1605-1615.

Stehlik, C., Krajewska, M., Welsh, K., Krajewski, S., Godzik, A., and Reed, J.C. (2003a) The PAAD/PYRIN-only protein POP1/ASC2 is a modulator of ASC-mediated NF-kB and pro-Caspase-1 regulation. Biochem. J., 373:101-113.

Stehlik, C., Lee, S.H., Dorfleutner, A., Stassinopoulos, A., Sagara, J., and Reed, J.C. (2003b) Apoptosis-associated speck-like protein containing a caspase recruitment domain is a regulator of procaspase-1 activation. J. Immunol., 171:6154-6163.

Stehlik, C. (2007) The PYRIN domain in signal transduction. Curr Protein Pept Sci.

Stehlik C, D.A. (2007) COPs and POPs: modulators of inflammasome activity. J Immunol, 179:7993-7998.

T Fernandes-Alnemri, J.W., J-W Yu, P Datta, B Miller1, W Jankowski, S Rosenberg, J Zhang and E S Alnemri. (2007) The pyroptosome: a supramolecular assembly of ASC dimers mediating inflammatory cell death via caspase-1 activation. Cell Death \& Differ, 14:1590-1604.

Taniguchi S, S.J. (2007) Regulatory molecules involved in inflammasome formation with special reference to a key mediator protein, ASC. seminars in immunopathology, 29:231-238.

Wang ET, S.R., Luo S, Khrebtukova I, Zhang L, Mayr C, Kingsmore SF, Schroth GP, Burge CB. (2008) Alternative isoform regulation in human tissue transcriptomes. Nature, 456:470-476.

Yamamoto, M., Yaginuma, K., Tsutsui, H., Sagara, J., Guan, X., Seki, E., Yasuda, K., Yamamoto, M., Akira, S., Nakanishi, K., Noda, T., and Taniguchi, S. (2004) ASC is essential for LPS-induced activation of procaspase-1 independently of TLR-associated signal adaptor molecules. Genes Cells, 9:1055-1067. 
Yu HB, F.B. (2008) The caspase-1 inflammasome: a pilot of innate immune responses. Cell Host and Microbe, 4:198-208. 


\section{General Discussion}

Physicians and scientists have long observed a connection between the process of inflammation and tumor development and progression. As early as the 1800's, Virchow proposed a direct link between inflammation and cancer based upon the enhanced proliferation observed in both disease states. Although increased cellular proliferation is not sufficient to induce cell transformation, Virchow's observation was not without merit. As our understanding of tumor cell biology and how it is impacted by inflammatory cytokines has evolved over the years, we have realized that these two processes are intimately intertwined. IL-1beta is an extremely potent pro-inflammatory cytokine, which has also been shown to participate at multiple levels of tumor progression including angiogenesis, recruitment of TAMs, and the upregulation of proliferation and survival genes (Apte, 2002,Apte RN, 2006a). Furthermore, the ability of IL-1beta to increase its own transcription via binding to IL-1R1 and subsequent NF-kB activation enhances its ability to sustain its production in the tumor microenvironment.

Recent studies in patients with autoinflammatory periodic fever syndromes have revealed how bypassing a key regulatory step in the production of mature IL-1beta can dramatically increase its production. In 2007, Gattorno et al., published an intriguing study examining IL-1beta secretion in monocytes from patients with disease-causing mutations in the NLRP3 gene. They determined 
that monocytes from these patients released greater amounts of IL-1 beta in response to inflammatory activation, which was not enhanced upon addition of ATP. These results suggest that there is already an elevated level of caspase-1 activation present in the monocytes from these patients. Interestingly, treatment of these patients with recombinant IL-1Ra (anakinra) in vivo, resulted in a decrease in the release of IL-1beta from their monocytes in vitro (Gattorno M, 2007). An independent research group also obtained similar results with this treatment (Goldbach-Mansky R, 2006). These results demonstrate that blocking the activity of IL-1 beta results in a decrease in further IL-1beta release and that is IL-1beta itself interacting with the IL-1R in an autocrine manner, which drives the prolonged inflammatory episodes. Therefore, dysregulation of the inflammasome can result in a self-sustaining increase in IL-1beta release in the tumor microenvironment, which can promote tumor progression and metastasis.

Through our cumulative studies we have showed that inflammasome activation and subsequent IL-1beta maturation is regulated through modulation of the adaptor protein ASC. First, we demonstrated that endogenous ASC must be redistributed from the nucleus to the cytoplasm in order for inflammasome assembly to take place. We provide the first evidence that the perinuclear aggregates formed by overexpression of ASC, do in fact occur on an endogenous level in response to inflammatory activation. Furthermore, these perinuclear aggregates incorporate other key inflammasome proteins including 
both caspase-1 and NLRP3 and therefore, are likely to represent assembled inflammasomes.

Our discovery that endogenous ASC is localized to the nucleus in resting monocytic cells raises the question of what function it may perform there. As we demonstrated in study 1 , the NLR receptors as well as IL-1 beta itself are confined to the cytoplasm. Therefore, any function carried out by ASC in the nucleus must be independent of inflammasome assembly. One possibility is that nuclear ASC modulates NF-kB activation. As mentioned above, several independent studies have linked ASC to NF-kB activity, although there is disagreement regarding whether it is an activator or an inhibitor of this important transcription factor (Masumoto, 2003,Stehlik, 2002,Taxman, 2006). Indeed, differences in NF-kB activity observed when expressing low levels of ASC versus high levels of ASC (Sarkar, 2006) may be due in part to localization of ASC as ASC is frequently nuclear when overexpressed at low levels and forms a cytoplasmic aggregate at higher levels (data not shown). This possible function of ASC in the nucleus is supported by our data showing co-localization between nuclear ASC and caspase-1, which also functions as a modulator of NF-kB activity. Therefore, further studies will need to be undertaken in order to address how the subcellular distribution of ASC impacts NF-kB activity.

The finding that the availability of cytoplasmic ASC serves as a key regulatory mechanism in inflammasome assembly is particularly intriguing in the context of cancer. It has been well documented that tumor cells of all types 
frequently secrete IL-1beta, and that inhibition of that secretion severely impairs their ability to grow and metastasize (Apte RN, 2006b,Voronov, 2003). However, as mentioned above, ASC is silenced by methylation in a large number of diverse tumors, presumably because of its role in pro-apoptotic pathways. Thus, in tumor cells, it appears that ASC performs dual functions, which are at odds with each other.

There are several possible mechanisms by which tumor cells may resolve the antagonistic roles of ASC. First, they may simply bypass the requirement for ASC by generating active caspase- 1 through another mechanism. Genetic destabilization may result in a large increase in the production of pro-caspase-1. As activation of caspase-1 is actually achieved through an induced proximity mechanism, a large increase in protein production could be sufficient to bring several molecules into close contact. Indeed, this is a phenomenon that we have observed in overexpression studies (data not shown). Alternatively, tumor cells could also release pro-IL-1beta, which would be subsequently processed by nonspecific extracellular proteases. This would bypass all regulatory mechanisms associated with inflammasome activation.

Further insight into how tumor cells resolve the antagonistic roles of ASC can be provided by recent studies, which examined when the methylationinduced silencing of ASC occurs. Studies in patients with colon and lung cancers found that silencing of ASC is a late-stage event (Machida, 2006,Riojas MA, 2007). Therefore, it is possible that tumor cells maintain ASC expression in early 
stages in order maintain their ability to release IL-1beta and subsequently recruit inflammatory cells such as macrophages to the tumor site. As the tumor progresses and the inflammatory infiltrate becomes established, the TAMs can then assume the responsibility of maintaining elevated levels of IL-1beta in the tumor microenvironment. This hypothesis is supported by immunohistochemistry data showing that in breast cancers, which have completely silenced ASC expression, it is still expressed in the surrounding inflammatory cells (Parsons MJ, 2009).

Our studies also revealed the existence of novel isoforms of ASC. We focused on how the structural modifications of ASC influenced the ability of each isoform to participate in inflammasome assembly. Of the three isoforms we identified, only one, $\mathrm{ASCb}$, retained was able to mediate inflammasome assembly, albeit with less efficiency than full-length ASCa. Intriguingly, in our search of the EST database, which led to the identification of these isoforms, we found that they were preferentially expressed in tumor tissue samples over the normal tissue couterparts. This suggests that expression of the ASC isoforms confers a selective advantage onto tumor cells. One possible explanation is that these isoforms provide an alternative mechanism by which tumor cells may escape the pro-apoptotic effects of ASC.

Our study did provide some evidence that these isoforms may be defective in their ability to participate in cell death. First, most studies of ASCinduced cell death have found that ASC localizes into a perinuclear aggregate 
prior to the cell's demise. This has been demonstrated for both apoptotic as well as pyroptotic forms of cell death (Masumoto, 1999,McConnell, 2000,T Fernandes-Alnemri, 2007). None of the isoforms we identified were capable of forming the characteristic 'speck' when overexpressed on their own. Although ASCb did aggregate in the presence of constitutively active NLRP3 and fulllength ASCa, the aggregates were larger and more irregularly shaped compared to those formed by ASCa. Furthermore, as previous studies examining the mechanism by which ASC induces apoptosis have found that the PYD is essential, the structural modifications observed in ASCc and ASCd are likely to render them incapable of participating in those pathways (Masumoto, 2003,Ohtsuka, 2004). Although ASCb possesses an intact PYD, its lack of a linker domain could make it less effective in mediating apoptosis, as was the case for its ability to mediate inflammasome assembly. Thus, further studies evaluating the capacity of each of these isoforms to function in cell death pathways are warranted.

The mounting evidence in favor of a role for IL-1beta in a number of disease processes including autoinflammatory disorders, CNS injury, in addition to cancer makes it an attractive therapeutic target. Indeed, anakinra is being evaluated for its ability to provide relief to patients suffering from FMF that is no longer responding to colchicine, gout, pseudo-gout, and the cryopyrinopathies with encouraging results (McGonagle D, 2007,McGonagle D, 2008,Roldan R, 2008 ,Ross JB, 2008,So A, 2007,Zhou, 1999). Furthermore, studies of in vivo 
tumor models have revealed that diminished IL-1beta secretion results in slower tumor growth and diminished invasiveness. Interestingly, the results are consistent regardless of whether it is the tumor cells or the host, which lack the capacity to release IL-1beta. Therefore, adjuvant treatment of cancer, particularly those that are associated with chronic inflammatory conditions such as gastric cancer, with anakinra or an IL-1beta neutralizing antibody may warrant future investigation.

In addition, the work presented here offers the potential for a new therapeutic target: inhibiting the nuclear export of ASC. As previously mentioned, tumor cells frequently target ASC for methylation-induced silencing. Therefore, complete neutralization of this protein would likely have protumorogenic effects. However, restricting ASC to the nucleus would prevent inflammasome assembly and subsequent IL-1beta processing without eliminating the protein. Furthermore, if the nuclear function of ASC involves the inhibition of NF-kB activity, then this method would an additional impact by preventing the transcription of multiple pro-survival and proliferative genes.

In summary, the work presented here that modulation of the inflammasome adaptor protein ASC through regulation of its subcellular localization and through alternative splicing can have significant impact on inflammasome assembly. We also identified Mycoplasma sp. as a novel activator of the inflammasome, which could have implications in the treatment of diseases caused by this organism as well as the inflammatory disorders that 
have been linked to it. Further exploration of the clinical relevance of ASC modulation as well as the potential for therapeutic targeting of these mechanisms is warranted. 


\section{General References}

A Rubartelli, F.C., M Talio, and R Sitia. (1990) A novel secretory pathway for interleukin-1 beta, a protein lacking a signal sequence. EMBO, 9:15031510.

Agostini, L., Martinon, F., Burns, K., McDermott, M.F., Hawkins, P.N., and Tschopp, J. (2004) NALP3 forms an IL-1 beta-processing inflammasome with increased activity in Muckle-Wells autoinflammatory disorder. Immunity, 20:319-325.

Akahira J, S.Y., Ito K, Niikura H, Okamura K, Yaegashi N. (2004) Promoter methylation status and expression of TMS1 gene in human epithelial ovarian cancer. Cancer Science, 95:40-43.

Apte, R.N., and Voronov, E. (2002) Interleukin-1--a major pleiotropic cytokine in tumor-host interactions. Semin Cancer Biol, 12:277-290.

Apte RN, D.S., Elkabets M, White MR, Reich E, Carmi Y, Song X, Dvozkin T, Krelin Y, Voronov E. (2006a) The involvement of IL-1 in tumorigenesis, tumor invasiveness, metastasis and tumor-host interactions. Cancer Metastasis Rev, 25:387-408.

Apte RN, K.Y., Song X, Dotan S, Recih E, Elkabets M, Carmi Y, Dvorkin T, White RM, Gayvoronsky L, Segal S, Voronov E. (2006b) Effects of microenvironment- and malignant cell-derived interleukin-1 in carcinogenesis, tumour invasiveness and tumour-host interactions. Eur J Cancer, 42:751759 .

Balci-Peynircioglu B, W.A., Hu C, Richards N, Staubach-Grosse A, Yilmaz E, Gumucio DL. (2008) Pyrin, product of the MEFV locus, interacts with the proapoptotic protein, Siva. Journal of Cell Physiology, 216:595-602.

Barksby HE, L.S., Preshaw PM, Taylor JJ. (2007) The expanding family of interleukin-1 cytokines and their role in destructive inflammatory disorders. Clin Exp Immunol, 149:217-225.

Becker CE, O.N.L. (2007) Inflammasomes in inflammatory disorders: the role of TLRs and their interactions with NLRs. Semin Immunopathol, 29:239-248. 
Bedoya F, S.L., Harton JA. (2007) Pyrin-only protein 2 modulates NF-kappaB and disrupts ASC:CLR interactions. Journal of Immunology, 178:38373845.

Bergsbaken T, F.S., Cookson BT. (2009) Pyroptosis: host cell death and inflammation. Nature Reviews Microbiology, 7 :99-109.

Bernot A, C.C., Dasilva C, Devaud C, Petit JL, Caloustian C, Cruaud C, Samson D, Pulcini F, Weissenbach J, Heilig R, Notanicola C, Domingo C, Rozenbaum M, Benchetrit E, Topaloglu R, Dewalle M, Dross C, Hadjari P, Dupont M, Demaille J, Touitou I, Smaoui N, Nedelec B, Méry JP, Chaabouni H, Delpech M, Grateau G. (1997) A candidate gene for familial Mediterranean fever. Nature Genetics, 17:25-31.

Braddock M, Q.A., Canvin J. (2004) Therapeutic potential of targeting IL-1 and IL-18 in inflammation. Expert Opin Biol Ther, 4:847-860.

Brough D, R.N. (2007) Caspase-1-dependent processing of pro-interleukin-1beta is cytosolic and precedes cell death. Journal of Cell Science, 120:772-781.

Bruey, J.M., Bruey-Sedano, N., Newman, R., Chandler, S., Stehlik, C., and Reed, J.C. (2004) PAN1/NALP2/PYPAF2, an inducible inflammatory mediator that regulates NF-kappaB and caspase-1 activation in macrophages. J. Biol. Chem., 279:51897-51907.

Cassel SL, E.S., lyer SS, Sadler JJ, Colegio OR, Tephly LA, Carter AB, Rothman PB, Flavell RA, Sutterwala FS. (2008) The Nalp3 inflammasome is essential for the development of silicosis. Proc Natl Acad Sci U S A, 105:9035-9040.

Chae, J.J., Komarow, H.D., Cheng, J., Wood, G., Raben, N., Liu, P.P., and Kastner, D.L. (2003) Targeted disruption of Pyrin, the FMF protein, causes heightened sensitivity to endotoxin and a defect in macrophage apoptosis. Mol. Cell., 11:591-604.

Chae, J.J., Wood, G., Masters, S.L., Richard, K., Park, G., Smith, B.J., and Kastner, D.L. (2006) The B30.2 domain of pyrin, the familial Mediterranean fever protein, interacts directly with caspase-1 to modulate IL-1beta production. Proc Natl Acad Sci U S A, 103:9982-9987.

Chae JJ, W.G., Richard K, Jaffe H, Colburn NT, Masters SL, Gumucio DL, Shoham NG, Kastner DL. (2008) The familial Mediterranean fever protein, pyrin, is cleaved by caspase-1 and activates NF-kappaB through its Nterminal fragment. Blood, 112:1794-1803. 
Collard RL, H.N., Monzon FA, Maier CE, O'Keefe DS. (2006) Methylation of the ASC gene promoter is associated with aggressive prostate cancer. Prostate, 66:687-695.

Conway, K.E., McConnell, B.B., Bowring, C.E., Donald, C.D., Warren, S.T., and Vertino, P.M. (2000) TMS1, a novel proapoptotic caspase recruitment domain protein, is a target of methylation-induced gene silencing in human breast cancers. Cancer Res., 60:6236-6242.

Coussens LM, W.Z. (2002) Inflammation and cancer. Nature, 420:860-867.

Cruz CM, R.A., Forman HJ, Ventura AL, Persechini PM, Ojcius DM. (2007) ATP activates a reactive oxygen species-dependent oxidative stress response and secretion of proinflammatory cytokines in macrophages. J Biol Chem, 282:2871-2879.

Dinarello, C.A. (1996) Biologic basis for interleukin-1 in disease. Blood, 87:20952147.

Dinarello CA, I.T., Warner SJ, Orencole SF, Lonnemann G, Cannon JG, Libby P. (1987) Interleukin 1 induces interleukin 1. I. Induction of circulating interleukin 1 in rabbits in vivo and in human mononuclear cells in vitro. $J$ Immunol, 139:1902-1910.

Dorfleutner, A., Bryan, N.B., Talbott, S.J., Funya, K.N., Rellick, S.L., Reed, J.C., Shi, X., Rojanasakul, Y., Flynn, D.C., and Stehlik, C. (2007a) Cellular PYRIN domain-only protein (cPOP) 2 is a candidate regulator of inflammasome activation. Infection and Immunity, in press.

Dorfleutner, A., McDonald, S.J., Bryan, N.B., Funya, K.N., Reed, J.C., Shi, X., Flynn, D.C., Rojanasakul, Y., and Stehlik, C. (2007b) A Shope Fibroma virus PYRIN-only protein modulates the host immune response. Virus Genes, in press.

Dowds, T.A., Masumoto, J., Zhu, L., Inohara, N., and Nunez, G. (2004) Cryopyrin-induced interleukin 1 beta secretion in monocytic cells: enhanced activity of disease-associated mutants and requirement for ASC. J. Biol. Chem., 279:21924-21928.

Druilhe, A., Srinivasula, S.M., Razmara, M., Ahmad, M., and Alnemri, E.S. (2001) Regulation of IL-1beta generation by Pseudo-ICE and ICEBERG, two dominant negative caspase recruitment domain proteins. Cell Death Differ., 8:649-657. 
Faustin B, L.L., Bruey JM, Luciano F, Sergienko E, Bailly-Maitre B, Volkmann N, Hanein D, Rouiller I, Reed JC. (2007) Reconstituted NALP1 inflammasome reveals two-step mechanism of caspase-1 activation. Molecular Cell, 25:713-724.

Fink SL, C.B. (2005) Apoptosis, pyroptosis, and necrosis: mechanistic description of dead and dying eukaryotic cells. Infection and Immunity, 73:1907-1916.

Fink SL, C.B. (2006) Caspase-1-dependent pore formation during pyroptosis leads to osmotic lysis of infected host macrophages. Cell Microbiol, 8:1812-1825.

Fonnesu C, C.C., Giovinale M, Curigliano V, Verrecchia E, de Socio G, La Regina M, Gasbarrini G, Manna R. (2008) Familial Mediterranean Fever: A review for clinical management. Joint Bone Spine.

Frisch, S.M., and Francis, H. (1994) Disruption of epithelial cell-matrix interactions induces apoptosis. J Cell Biol, 124:619-626.

Fujisawa A, K.N., Saito M, Nishikomori R, Tanizaki H, Kanazawa N, Adachi S, Heike T, Sagara J, Suda T, Nakahata T, Miyachi Y. (2007) Diseaseassociated mutations in CIAS1 induce cathepsin B-dependent rapid cell death of human THP-1 monocytic cells. Blood, 109:2903-2911.

Galán JE, W.-W.H. (2006) Protein delivery into eukaryotic cells by type III secretion machines. Nature, 444:567-573.

Gattorno M, T.S., Carta S, Delfino L, Ferlito F, Pelagatti MA, D'Osualdo A, Buoncompagni A, Alpigiani MG, Alessio M, Martini A, Rubartelli A. (2007) Pattern of interleukin-1 beta secretion in response to lipopolysaccharide and ATP before and after interleukin-1 blockade in patients with CIAS1 mutations. Arthritis Rheum, 56:3138-3148.

Goldbach-Mansky R, D.N., Canna SW, Gelabert A, Jones J, Rubin BI, Kim HJ, Brewer C, Zalewski C, Wiggs E, Hill S, Turner ML, Karp BI, Aksentijevich I, Pucino F, Penzak SR, Haverkamp MH, Stein L, Adams BS, Moore TL, Fuhlbrigge RC, Shaham B, Jarvis JN, O'Neil K, Vehe RK, Beitz LO, Gardner G, Hannan WP, Warren RW, Horn W, Cole JL, Paul SM, Hawkins PN, Pham TH, Snyder C, Wesley RA, Hoffmann SC, Holland SM, Butman JA, Kastner DL. (2006) Neonatal-onset multisystem inflammatory disease responsive to interleukin-1beta inhibition. $\mathrm{N}$ Engl $\mathrm{J}$ Med, 355:581-592. 
Guan, X., Sagara, J., Yokoyama, T., Koganehira, Y., Oguchi, M., Saida, T., and Taniguchi, S. (2003) ASC/TMS1, a caspase-1 activating adaptor, is downregulated by aberrant methylation in human melanoma. Int. J. Cancer, 107:202-208.

Halle A, H.V., Petzold GC, Stewart CR, Monks BG, Reinheckel T, Fitzgerald KA, Latz E, Moore KJ, Golenbock DT. (2008) The NALP3 inflammasome is involved in the innate immune response to amyloid-beta. Nat Immunol, 9:857-865.

Hasegawa, M., Imamura, R., Kinoshita, T., Matsumoto, N., Masumoto, J., Inohara, N., and Suda, T. (2005) ASC-mediated NF-kappaB activation leading to interleukin-8 production requires caspase- 8 and is inhibited by CLARP. J. Biol. Chem., 280:15122-15130.

Hornung V, B.F., Halle A, Samstad EO, Kono H, Rock KL, Fitzgerald KA, Latz E. (2008) Silica crystals and aluminum salts activate the NALP3 inflammasome through phagosomal destabilization. Nature Immunology, 9:847-856.

Howard AD, K.M., Thornberry N, Ding GJ, Limjuco G, Weidner J, Salley JP, Hogquist KA, Chaplin DD, Mumford RA, . (1991) IL-1-converting enzyme requires aspartic acid residues for processing of the IL-1 beta precursor at two distinct sites and does not cleave 31-kDa IL-1 alpha. Journal of Immunology, 147:2964-2969.

Humke, E.W., Shriver, S.K., Starovasnik, M.A., Fairbrother, W.J., and Dixit, V.M. (2000) ICEBERG: a novel inhibitor of interleukin-1beta generation. Cell, 103:99-111.

Johnston JB, B.J., Nazarian SH, Goodwin M, Ricciuto D, Wang G, McFadden G. (2005) A poxvirus-encoded pyrin domain protein interacts with ASC-1 to inhibit host inflammatory and apoptotic responses to infection. Immunity, 23:587-598.

Juhas M, C.D., Hood DW. (2008) Type IV secretion systems: tools of bacterial horizontal gene transfer and virulence. Cell Microbiol, 10:2377-2386.

Kanneganti, T.D., Body-Malapel, M., Amer, A., Park, J.H., Whitfield, J., Taraporewala, Z.F., Miller, D., Patton, J.T., Inohara, N., and Nunez, G. (2006a) Critical role for cryopyrin/Nalp3 in activation of caspase-1 in response to viral infection and double-stranded RNA. J Biol Chem. 
Kanneganti, T.D., Ozoren, N., Body-Malapel, M., Amer, A., Park, J.H., Franchi, L., Whitfield, J., Barchet, W., Colonna, M., Vandenabeele, P., Bertin, J., Coyle, A., Grant, E.P., Akira, S., and Nunez, G. (2006b) Bacterial RNA and small antiviral compounds activate caspase-1 through cryopyrin/Nalp3. Nature, 440:232-236.

Kanneganti TD, L.M., Kim YG, Chen G, Park JH, Franchi L, Vandenabeele P, Núñez G. (2007 ) Pannexin-1-mediated recognition of bacterial molecules activates the cryopyrin inflammasome independent of Toll-like receptor signaling. Immunity, 26:433-443.

Karin, M., and Ben-Neriah, Y. (2000) Phosphorylation meets ubiquitination: The control of NF-kB activity. Ann. Rev. Immunol., 18:621-663.

Kastner, D.L. (2005) Hereditary periodic Fever syndromes. Hematology (Am Soc Hematol Educ Program):74-81.

Keller M, R.A., Werner S, Beer HD. (2008) Active caspase-1 is a regulator of unconventional protein secretion. Cell, 132:818-831.

Kimura YN, W.K., Fotovati A, Hosoi F, Yasumoto K, Izumi H, Kohno K, Umezawa K, Iguchi H, Shirouzu K, Takamori S, Kuwano M, Ono M. (2007) Inflammatory stimuli from macrophages and cancer cells synergistically promote tumor growth and angiogenesis. Cancer Science, 98:2009-2018.

Knudson, A. (1971) Mutation and cancer: statistical study of retinoblastoma. Proc Natl Acad Sci U S A, 68 :820-823.

Krelin, Y., Voronov, E., Dotan, S., Elkabets, M., Reich, E., Fogel, M., Huszar, M., Iwakura, Y., Segal, S., Dinarello, C.A., and Apte, R.N. (2007) Interleukin1 beta-driven inflammation promotes the development and invasiveness of chemical carcinogen-induced tumors. Cancer Res, 67:1062-1071.

Lamkanfi, M., Denecker, G., Kalai, M., D'Hondt, K., Meeus, A., Declercq, W., Saelens, X., and Vandenabeele, P. (2004) INCA, a novel human caspase recruitment domain protein that inhibits interleukin-1beta generation. J. Biol. Chem., 279:51729-51738.

Lamkanfi M, K.M., Saelens X, Declercq W, Vandenabeele P. (2004) Caspase-1 activates nuclear factor of the kappa-enhancer in B cells independently of its enzymatic activity. Journal of Biological chemistry, 279:24785-24793. 
Lee, S.-H., Stehlik, C., and Reed, J.C. (2001) COP, a CARD-containing protein and inhibitor of pro-interleukin-1b processing. J Biol Chem, 276:3449534500 .

Levin TC, W.K., Leppla SH, Moayeri M. (2008) Heat shock inhibits caspase-1 activity while also preventing its inflammasome-mediated activation by anthrax lethal toxin. Cell Microbiol, 10:2434-2446.

Li, P., Allen, H., Banerjee, S., Franklin, S., Herzog, L., Johnston, C., McDowell, J., Paskind, M., Rodman, L., Salfeld, J., Towne, E., Tracey, D., Wardwell, S., Wei, F.-Y., Wong, W., et al. (1995) Mice deficient in IL-1b-converting enzyme are defective in production of mature IL-1b and resistant to endotoxic shock. Cell, 80:401-411.

Liepinsh, E., Barbals, R., Dahl, E., Sharipo, A., Staub, E., and Otting, G. (2003) The death-domain fold of the ASC PYRIN domain, presenting a basis for PYRIN/PYRIN recognition. J. Mol. Biol., 332:1155-1163.

Locovei S, S.E., Qiu F, Spray DC, Dahl G. (2007) Pannexin1 is part of the pore forming unit of the P2X(7) receptor death complex. FEBS Lett, 581:483488.

Machida, E.O., Brock, M.V., Hooker, C.M., Nakayama, J., Ishida, A., Amano, J., Picchi, M.A., Belinsky, S.A., Herman, J.G., Taniguchi, S., and Baylin, S.B. (2006) Hypermethylation of ASC/TMS1 is a sputum marker for late-stage lung cancer. Cancer Res, 66:6210-6218.

Mao, P.-L., Jiang, Y., Wee, B., and Porter, A. (1998) Activation of caspase-1 in the nucleus requires nuclear translocation of pro-caspase-1 mediated by its prodomain. J. Biol. Chem., 273:23621-23624.

Mariathasan, S., Newton, K., Monack, D.M., Vucic, D., French, D.M., Lee, W.P., Roose-Girma, M., Erickson, S., and Dixit, V.M. (2004) Differential activation of the inflammasome by caspase-1 adaptors ASC and Ipaf. Nature, 430:213-218.

Mariathasan, S., Weiss, D.S., Newton, K., McBride, J., O'Rourke, K., RooseGirma, M., Lee, W.P., Weinrauch, Y., Monack, D.M., and Dixit, V.M. (2006) Cryopyrin activates the inflammasome in response to toxins and ATP. Nature, 440:228-232.

Marina-García N, F.L., Kim YG, Miller D, McDonald C, Boons GJ, Núñez G. (2008) Pannexin-1-mediated intracellular delivery of muramyl dipeptide 
induces caspase-1 activation via cryopyrin/NLRP3 independently of Nod2. Journal of Immunology, 180:4050-4057.

Marriott HM, M.T., Dockrell DH. (2008) Pneumolysin: a double-edged sword during the host-pathogen interaction. Curr Mol Med, 8:497-509.

Martinon, F., Burns, K., and Tschopp, J. (2002) The Inflammasome: A molecular platform triggering activation of inflammatory caspases and processing of prolL-b. Mol. Cell, 10:417-426.

Martinon, F., Agostini, L., Meylan, E., and Tschopp, J. (2004) Identification of bacterial muramyl dipeptide as activator of the NALP3/cryopyrin inflammasome. Curr Biol, 14:1929-1934.

Martinon, F., Petrilli, V., Mayor, A., Tardivel, A., and Tschopp, J. (2006) Goutassociated uric acid crystals activate the NALP3 inflammasome. Nature, 440:237-241.

Masumoto, J., Taniguchi, S., Ayukawa, K., Sarvotham, H., Kishino, T., Niikawa, N., Hidaka, E., Katsuyama, T., Higuchi, T., and Sagara, J. (1999) ASC, a novel 22-kDa protein, aggregates during apoptosis of human promyelocytic leukemia HL-60 cells. J. Biol. Chem., 274:33835-33838.

Masumoto, J., Taniguchi, S., and Sagara, J. (2001a) Pyrin N-terminal homology domain- and caspase recruitment domain-dependent oligomerization of ASC. Biochem. Biophysical. Res. Commun., 280:652-655.

Masumoto, J., Taniguchi, S.-I., Nakayama, J., Shiohara, M., Hidaka, E., Katsuyama, T., Murase, S., and Sagara, J. (2001b) Expression of apoptosis-associated speck-like protein containing a caspase recruitment domain, a Pyrin N-terminal homology domain-containing protein , in normal human tissues. J. Histochem. Cytochem., 49:1269-1275.

Masumoto, J., Dowds, T.A., Schaner, P., Chen, F.F., Ogura, Y., Li, M., Zhu, L., Katsuyama, T., Sagara, J., Taniguchi, S., Gumucio, D.L., Nunez, G., and Inohara, N. (2003) ASC is an activating adaptor for NF-kappaB and caspase-8-dependent apoptosis. Biochem. Biophys. Res. Commun., 303:69-73.

McConnell, B.B., and Vertino, P.M. (2000) Activation of a caspase-9-mediated apoptotic pathway by subcellular redistribution of the novel caspase recruitment domain protein TMS1. Cancer Res., 60:6243-6247. 
McGonagle D, T.A., Madden J, Emery P, McDermott MF. (2008) Successful treatment of resistant pseudogout with anakinra. Arthritis Rheum, 58:631633.

McGonagle D, T.A., Shankaranarayana S, Madden J, Emery P, McDermott MF. (2007) Management of treatment resistant inflammation of acute on chronic tophaceous gout with anakinra. Ann Rheum Dis, 66:1683-1684.

Miao EA, A.-A.C., Dors M, Clark AE, Bader MW, Miller SI, Aderem A. (2006) Cytoplasmic flagellin activates caspase- 1 and secretion of interleukin 1 beta via Ipaf. Nat Immunol, 7:569-575.

Moriya, M., Taniguchi, S., Wu, P., Liepinsh, E., Otting, G., and Sagara, J. (2005) Role of charged and hydrophobic residues in the oligomerization of the PYRIN domain of ASC. Biochemistry, 44:575-583.

Muruve DA, P.V., Zaiss AK, White LR, Clark SA, Ross PJ, Parks RJ, Tschopp J. (2008) The inflammasome recognizes cytosolic microbial and host DNA and triggers an innate immune response. Nature, 452:103-107.

Nakagawara A, N.Y., Ikeda H, Hiwasa T, Kuida K, Su MS, Zhao H, Cnaan A, Sakiyama S. (1997) High levels of expression and nuclear localization of interleukin-1 beta converting enzyme (ICE) and CPP32 in favorable human neuroblastomas. Cancer Research, 57:4578-4584.

Netea MG, N.-P.C., Nold MF, Joosten LA, Opitz B, van der Meer JH, van de Veerdonk FL, Ferwerda G, Heinhuis B, Devesa I, Funk CJ, Mason RJ, Kullberg BJ, Rubartelli A, van der Meer JW, Dinarello CA. (2009) Differential requirement for the activation of the inflammasome for processing and release of IL-1beta in monocytes and macrophages. Blood, 113:2324-2335.

Netea MG, v.d.V.F., Kullberg BJ, Van der Meer JW, Joosten LA. (2008) The role of NLRs and TLRs in the activation of the inflammasome. Expert Opin Biol Ther, 8:1867-1872.

Ohtsuka, T., Ryu, H., Minamishima, Y.A., Macip, S., Sagara, J., Nakayama, K.I., Aaronson, S.A., and Lee, S.W. (2004) ASC is a Bax adaptor and regulates the p53-Bax mitochondrial apoptosis pathway. Nat. Cell. Biol., 6:121-128.

Ono, M. (2008) Molecular links between tumor angiogenesis and inflammation: inflammatory stimuli of macrophages and cancer cells as targets for therapeutic strategy. Cancer Science, 99:1501-1506. 
Papin S, C.S., Agostini L, Martinon F, Werner S, Beer HD, Grütter C, Grütter M, Tschopp J. (2007) The SPRY domain of Pyrin, mutated in familial Mediterranean fever patients, interacts with inflammasome components and inhibits prolL-1beta processing. Cell Death \& Differ, 14:1457-1466.

Parsons MJ, P.P., Brat DJ, Colbert L, Vertino PM. (2009) Silencing of TMS1/ASC promotes resistance to anoikis in breast epithelial cells. Cancer Research, 69:1706-1711.

Parsons MJ, V.P. (2006) Dual role of TMS1/ASC in death receptor signaling. Oncogene, 25:6948-6958.

Partha M Das, K.R., Jane VanWert, Larry Ferdinand, Gopal Gopisetty, Isildinha M Reis and Rakesh Singal. (2006) Methylation mediated silencing of TMS1/ASC gene in prostate cancer. Molecular Cancer, 5.

Pelegrin P, S.A. (2006) Pannexin-1 mediates large pore formation and interleukin-1 beta release by the ATP-gated P2X7 receptor. EMBO, 25:5071-5082.

Perkins, N.D. (2004) NF-kB: tumor promoter or suppressor? Trends Cell Biol., 14:64-69.

Pétrilli V, P.S., Dostert C, Mayor A, Martinon F, Tschopp J. (2007) Activation of the NALP3 inflammasome is triggered by low intracellular potassium concentration. Cell Death \& Differ, 14:1583-1589.

Piccini A, C.S., Tassi S, Lasiglié D, Fossati G, Rubartelli A. (2008) ATP is released by monocytes stimulated with pathogen-sensing receptor ligands and induces IL-1beta and IL-18 secretion in an autocrine way. Proc Natl Acad Sci U S A, 105:8067-8072.

Qian X, H.C., Cho CH, Hui WM, Rashid A, Chan AO. (2008) E-cadherin promoter hypermethylation induced by interleukin-1beta treatment or $\mathrm{H}$. pylori infection in human gastric cancer cell lines. Cancer Letters, 263:107-113.

Richards, N., Schaner, P., Diaz, A., Stcukey, J., Shelden, E., Wadhwa, A., and Gumucio, D.L. (2001) Interaction between Pyrin and the apoptotic speck protein (ASC) modulates ASC-induced apoptosis. J. Biol. Chem., 276:39320-39329.

Riojas MA, G.M., Glöckner SC, Machida EO, Baylin SB, Ahuja N. (2007) Methylation-induced silencing of ASC/TMS1, a pro-apoptotic gene, is a late-stage event in colorectal cancer. Cancer Biol. Ther., 6:1710-1716. 
Roldan R, R.A., Miranda MD, Collantes E. (2008) Anakinra: new therapeutic approach in children with Familial Mediterranean Fever resistant to colchicine. Joint Bone Spine, 75:504-505.

Ross JB, F.L., Klotz PJ, Langley RG, Gaudet R, Thompson K, Churchman SM, McDermott MF, Hawkins PN. (2008) Use of anakinra (Kineret) in the treatment of familial cold autoinflammatory syndrome with a 16-month follow-up. J Cutan Med Surg, 12:8-16.

Saleh M, M.J., Wolinski MK, Bensinger SJ, Fitzgerald P, Droin N, Ulevitch RJ, Green DR, Nicholson DW. (2006) Enhanced bacterial clearance and sepsis resistance in caspase-12-deficient mice. Nature, 440:1064-1068.

Samuels, J., and Ozen, S. (2006) Familial Mediterranean fever and the other autoinflammatory syndromes: evaluation of the patient with recurrent fever. Curr Opin Rheumatol, 18:108-117.

Sarkar, A., Duncan, M., Hart, J., Hertlein, E., Guttridge, D.C., and Wewers, M.D. (2006) ASC Directs NF-\{kappa\}B Activation by Regulating Receptor Interacting Protein-2 (RIP2) Caspase-1 Interactions. J Immunol, 176:49794986.

Schumann, R.R., Belka, C., Reuter, D., Lamping, N., Kirschning, C.J., Weber, J.R., and Pfeil, D. (1998) Lipopolysaccharide activates caspase-1 (interleukin-1-converting enzyme) in cultured monocytic and endothelial cells. Blood, 91:577-584.

Seshadri S, D.M., Hart JM, Gavrilin MA, Wewers MD. (2007) Pyrin levels in human monocytes and monocyte-derived macrophages regulate IL-1beta processing and release. Journal of Immunology, 179:1274-1281.

Shiohara, M., Taniguchi, S., Masumoto, J., Yasui, K., Koike, K., Komiyama, A., and Sagara, J. (2002) ASC, which is composed of a PYD and a CARD, is up-regulated by inflammation and apoptosis in human neutrophils. Biochem. Biophys. Res. Commun., 293:1314-1318.

Siegel, R., Martin, D., Zheng, L., Ng, S., Bertin, J., Cohen, J., and Lenardo, M. (1998) Death-effector filaments: novel cytoplasmic structures that recruit caspases and trigger apoptosis. J. Cell Biol., 141:1243-1253.

So A, D.S.T., Revaz S, Tschopp J. (2007) A pilot study of IL-1 inhibition by anakinra in acute gout. Arthritis Res Ther, 9. 
Solle M, L.J., Perregaux DG, Stam E, Petrushova N, Koller BH, Griffiths RJ, Gabel CA. (2001) Altered cytokine production in mice lacking P2X(7) receptors. Journal of Biological Chemistry, 276:125-132.

Srimathi T, R.S., Dubas RL, Chang H, Cheng H, Roder H, Park YC. (2008) Mapping of POP1-binding site on pyrin domain of ASC. Journal of Biological Chemistry, 283:15390-15398.

Srinivasula, S.M., Poyet, J.-L., Razmara, M., Datta, P., Zhang, Z., and Alnemri, E.S. (2002) The PYRIN-CARD protein ASC is an activating adaptor for Caspase-1. J. Biol. Chem., 277:21119-21122.

Stehlik, C., Fiorentino, L., Dorfleutner, A., Bruey, J.M., Ariza, E.M., Sagara, J., and Reed, J.C. (2002) The PAAD/PYRIN-family protein ASC is a dual regulator of a conserved step in nuclear factor kappaB activation pathways. J. Exp. Med., 196:1605-1615.

Stehlik, C., Krajewska, M., Welsh, K., Krajewski, S., Godzik, A., and Reed, J.C. (2003a) The PAAD/PYRIN-only protein POP1/ASC2 is a modulator of ASC-mediated NF-kB and pro-Caspase-1 regulation. Biochem. J., 373:101-113.

Stehlik, C., Lee, S.H., Dorfleutner, A., Stassinopoulos, A., Sagara, J., and Reed, J.C. (2003b) Apoptosis-associated speck-like protein containing a caspase recruitment domain is a regulator of procaspase-1 activation. J. Immunol., 171:6154-6163.

Stehlik C, D.A. (2007) COPs and POPs: modulators of inflammasome activity. J Immunol, 179:7993-7998.

Stone, A.R., Bobo, W., Brat, D.J., Devi, N.S., Van Meir, E.G., and Vertino, P.M. (2004) Aberrant methylation and down-regulation of TMS1/ASC in human glioblastoma. Am. J. Pathol., 165:1151-1161.

Sutterwala, F.S., Ogura, Y., Szczepanik, M., Lara-Tejero, M., Lichtenberger, G.S., Grant, E.P., Bertin, J., Coyle, A.J., Galan, J.E., Askenase, P.W., and Flavell, R.A. (2006) Critical role for NALP3/CIAS1/Cryopyrin in innate and adaptive immunity through its regulation of caspase-1. Immunity, 24:317327.

Suzuki T, F.L., Toma C, Ashida H, Ogawa M, Yoshikawa Y, Mimuro H, Inohara N, Sasakawa C, Nuñez G. (2007) Differential regulation of caspase-1 activation, pyroptosis, and autophagy via Ipaf and ASC in Shigellainfected macrophages. PLoS Pathogens, 3:1082-1091. 
T Fernandes-Alnemri, J.W., J-W Yu, P Datta, B Miller1, W Jankowski, S Rosenberg, J Zhang and E S Alnemri. (2007) The pyroptosome: a supramolecular assembly of ASC dimers mediating inflammatory cell death via caspase-1 activation. Cell Death \& Differ, 14:1590-1604.

Taxman, D.J., Zhang, J., Champagne, C., Bergstralh, D.T., locca, H.A., Lich, J.D., and Ting, J.P. (2006) Cutting edge: ASC mediates the induction of multiple cytokines by Porphyromonas gingivalis via caspase-1-dependent and -independent pathways. J Immunol, 177:4252-4256.

Terasawa, K., Sagae, S., Toyota, M., Tsukada, K., Ogi, K., Satoh, A., Mita, H., Imai, K., Tokino, T., and Kudo, R. (2004) Epigenetic inactivation of TMS1/ASC in ovarian cancer. Clin. Cancer Res., 10:2000-2006.

Thomas PG, D.P., Aldridge JR Jr, Ellebedy AH, Reynolds C, Funk AJ, Martin WJ, Lamkanfi M, Webby RJ, Boyd KL, Doherty PC, Kanneganti TD. (2009) The intracellular sensor NLRP3 mediates key innate and healing responses to influenza A virus via the regulation of caspase-1. Immunity, 30:566-575.

Thornberry, N.A., Bull, H.G., Calaycay, J.R., Chapman, K.T., Howard, A.D., Kostura, M.J., Miller, D.K., Molineaux, S.M., Weidner, J.R., Aunins, J., Elliston, K.O., Ayala, J.M., Casanoparallel, F.J., Chin, J., Ding, G.J.F., et al. (1992) A novel heterodimeric cysteine protease is required for interleukin-1 beta processing in monocytes. Nature, 356:768-774.

Tschopp, J., Martinon, F., and Burns, K. (2003) NALPs: a novel protein family involved in inflammation. Nat. Rev. Mol. Cell. Biol., 4:95-104.

Tu S, B.G., Cui G, Takaishi S, Kurt-Jones EA, Rickman B, Betz KS, PenzOesterreicher M, Bjorkdahl O, Fox JG, Wang TC. (2008) Overexpression of interleukin-1 beta induces gastric inflammation and cancer and mobilizes myeloid-derived suppressor cells in mice. Cancer Cell, 14:408419.

Virmani, A., Rathi, A., Sugio, K., Sathyanarayana, U.G., Toyooka, S., Kischel, F.C., Tonk, V., Padar, A., Takahashi, T., Roth, J.A., Euhus, D.M., Minna, J.D., and Gazdar, A.F. (2003) Aberrant methylation of TMS1 in small cell, non small cell lung cancer and breast cancer. Int. J. Cancer, 106:198-204.

Voronov, E., Shouval, D.S., Krelin, Y., Cagnano, E., Benharroch, D., Iwakura, Y., Dinarello, C.A., and Apte, R.N. (2003) IL-1 is required for tumor 
invasiveness and angiogenesis. Proc Natl Acad Sci U S A, 100:26452650.

Waite AL, S.P., Hu C, Richards N, Balci-Peynircioglu B, Hong A, Fox M, Gumucio DL. (2009) Pyrin and ASC co-localize to cellular sites that are rich in polymerizing actin. Experimental Biology and Medicine, 234:40-52.

Warner, S.J., Auger, K.R., and Libby, P. (1987) Interleukin 1 induces interleukin 1. II. Recombinant human interleukin 1 induces interleukin 1 production by adult human vascular endothelial cells. J Immunol, 139:1911-1917.

Yamamoto, M., Yaginuma, K., Tsutsui, H., Sagara, J., Guan, X., Seki, E., Yasuda, K., Yamamoto, M., Akira, S., Nakanishi, K., Noda, T., and Taniguchi, S. (2004) ASC is essential for LPS-induced activation of procaspase-1 independently of TLR-associated signal adaptor molecules. Genes Cells, 9:1055-1067.

Yokoyama, T., Sagara, J., Guan, X., Masumoto, J., Takeoka, M., Komiyama, Y., Miyata, K., Higuchi, K., and Taniguchi, S. (2003) Methylation of ASC/TMS1, a proapoptotic gene responsible for activating procaspase-1, in human colorectal cancer. Cancer Lett., 202:101-108.

Yu, J.W., Wu, J., Zhang, Z., Datta, P., Ibrahimi, I., Taniguchi, S., Sagara, J., Fernandes-Alnemri, T., and Alnemri, E.S. (2006) Cryopyrin and pyrin activate caspase-1, but not NF-kappaB, via ASC oligomerization. Cell Death Differ, 13:236-249.

Zhang C, L.H., Zhou G, Zhang Q, Zhang T, Li J, Zhang J, Hou J, Liew CT, Yin D. (2007) Transcriptional silencing of the TMS1/ASC tumour suppressor gene by an epigenetic mechanism in hepatocellular carcinoma cells. Journal of Pathology, 212:134-142.

Zhang Z, T.S., Zhang L. (2006) Prognostic value of apoptosis-associated specklike protein containing a CARD gene promoter methylation in resectable non-small-cell lung cancer. Clinical Lung Cancer, 8:62-65.

Zhou, P., Chou, J., Olea, R.S., Yuan, J., and Wagner, G. (1999) Solution structure of Apaf-1 CARD and its interaction with caspase-9 CARD: a structural basis for specific adaptor/caspase interaction. Proc. Natl. Acad. Sci. U.S.A., 96:11265-11270. 